Faculdade de Engenharia da Universidade do Porto

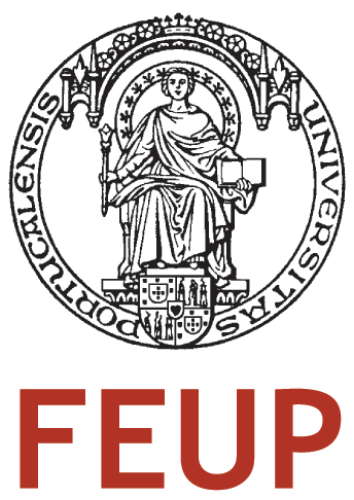

\title{
Phone Based Fall Risk Prediction
}

\author{
Vânia Margarida Cardoso Guimarães
}

Master in Bioengineering

Supervisor: Miguel Pimenta Monteiro (PhD) 



\title{
Phone Based Fall Risk Prediction
}

\author{
Vânia Margarida Cardoso Guimarães
}

Master in Bioengineering

Approved in oral examination by the committee:

Chair: Artur Cardoso (PhD)

External Examiner: Jorge Alves da Silva ( $\mathrm{PhD})$

Supervisor at FhP: Dirk Elias (PhD)

Supervisor at FEUP: António Pimenta Monteiro (PhD)

$12^{\text {th }}$ July, 2011 



\section{Abstract}

Falls in older people can represent serious individual and socioeconomic problems. With the increasing number of older people in the population, management of falls is becoming a challenge.

It is now recognized that several risk factors for falling can be identified, and specific interventions can be designed in order to reduce those risks. Several tools were then developed and are now available to screen for fall risk. However, these tools are applied with low frequency, usually requiring the use of expensive equipments that can only be manipulated by experts. This poses some constraints on the prevention of falls, since when these techniques are applied, it can be too late.

In order to improve the earlier detection of risks and the application of fall prevention strategies, new community based solutions must be developed. In this project, the mobile phone was purposed as a potential instrument to perform fall risk screening. Several strategies were then evaluated to be adapted to the phone for fall risk screening purposes.

Phone's inertial sensors were used to extract some parameters of gait that were related with an increased risk of falling. Foot contacts, gait phases, step length, step duration, walking velocity and variability parameters could then be determined from sensors data. Acceptable measures of step length and walking velocity could be obtained. Additionally, other risk factors were selected to be assessed by self-report, using some clinical scales/questionnaires.

Results suggest that phone can be used as a major strategy to improve fall prevention, being a great value for older persons and society. 


\section{Resumo}

Uma queda num idoso pode resultar em graves problemas individuais e socioeconómicos. Com o aumento do número de idosos na população, a gestão de quedas está a tornar-se um desafio.

Actualmente, é possível identificar vários factores de risco para a ocorrência de quedas bem como reduzir esses riscos, através da aplicação da aplicação de intervenções específicas. Nesse sentido, há já algumas ferramentas actualmente disponíveis para avaliar o risco de queda. Estas ferramentas no entanto usadas com baixa frequência, exigindo o uso de equipamentos caros que apenas podem ser manipulados por pessoas qualificadas. Muitas vezes, estas técnicas podem já ser aplicadas tarde demais.

Para que a detecção dos riscos e a aplicação de estratégias de prevenção de quedas possa ser feita mais precocemente, é necessário o desenvolvimento de novas soluções dirigidas à população. Neste projecto, foi proposto o uso do telemóvel para a detecção do risco de queda nos idosos.

Os sinais dos sensores do telemóvel foram utilizados para extrair parâmetros da marcha relacionados com um risco de queda aumentado. A partir deles, os contactos do pé com o chão, as fases da marcha, o tamanho e duração dos passos, a velocidade e parâmetros que quantificam a variabilidade da marcha puderam ser determinados. Medidas aceitáveis do tamanho dos passos e da velocidade foram obtidas. Adicionalmente, outros factores de risco foram seleccionados para serem reportados pela própria pessoa, usando algumas escalas ou questionários clínicos.

Os resultados do projecto sugerem que o telemóvel pode ser usado como uma maisvalia no melhoramento da prevenção das quedas, sendo também uma mais-valia para as pessoas idosas e para a sociedade. 


\section{Acknowledgements}

Prof. Doctor Miguel Pimenta Monteiro, my supervisor at FEUP, provided prompt support during different phases of project and useful recommendations on documents writing.

Prof. Doctor Dirk Elias, my primary supervisor at Fraunhofer AICOS Portugal, shared me his enthusiasm on the project, gave important insights on its importance and useful recommendations to consider on its development.

Pedro Teixeira, researcher at Fraunhofer AICOS Portugal, guided me in all phases of the project. He advised me with important research lines that truly helped on the progression of the work and gave important insights on how to solve problems.

All the people at Frunhofer AICOS Portugal, who promptly participated on the walking tests. 


\section{Contents}

1 Introduction 1

1.1 The problem .......................... 2

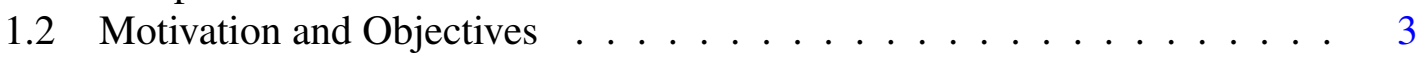

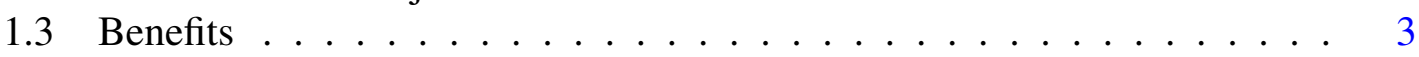

1.4 Report's structure ...................... 4

2 Background and Literature Review 5

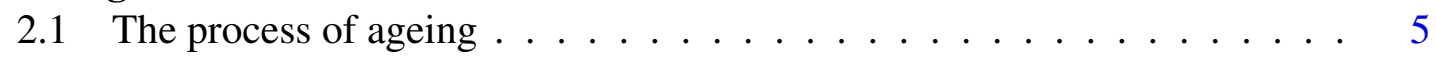

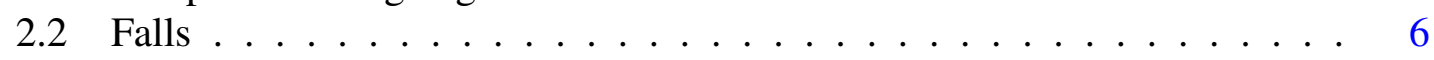

2.2.1 Definitions .................... 6

2.2 .2 Aetiology ..................... 7

2.2 .3 Incidence . . . . . . . . . . . . . . . 8

2.2 .4 Impact $\ldots \ldots \ldots \ldots$

2.3 Risk factors for falls . . . . . . . . . . . . . . . . . . 9

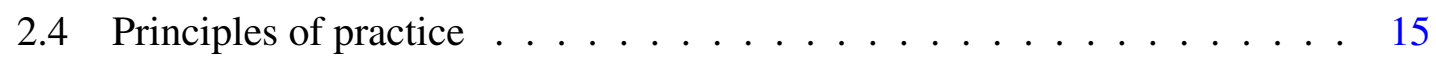

2.4.1 Current Guidelines . . . . . . . . . . . . . . . 15

2.5 Clinical tools/technologies . . . . . . . . . . . . . . . . 19

2.5.1 Fall risk screening tools . . . . . . . . . . . . . 20

2.5.2 Fall risk assessment tools . . . . . . . . . . . . . 23

2.6 Falls prevention . . . . . . . . . . . . . . . 25

2.7 Recent studies on fall risk prediction . . . . . . . . . . . . . 26

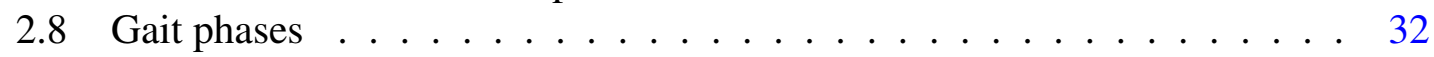

2.9 Using inertial sensors to evaluate gait $\ldots \ldots \ldots 33$

2.9.1 Sensors signal analysis . . . . . . . . . . . . . . 33

2.9.2 Gait parameters . . . . . . . . . . . . . . . . . . 35

2.10 Smartphones and Android . . . . . . . . . . . . . . . . . 44

2.11 Mobile phones and older people . . . . . . . . . . . . . 46

3 Fall Risk Screening Method 49

3.1 Hypothesis and projects . . . . . . . . . . . . . . . . . . 49

3.2 Inertial sensors signal recording . . . . . . . . . . . . . 51

3.3 Data Analysis and Events Detection . . . . . . . . . . . 52

3.3.1 Trial tests recording . . . . . . . . . . . . 52

3.3.2 Foot contacts detection . . . . . . . . . . . . . 53

3.3.3 Right $v s$. Left foot contacts discrimination . . . . . . . . . . . 55

3.3 .4 Toe offs detection . . . . . . . . . . . . . . . 56

3.3 .5 Gait Phases . . . . . . . . . . . . . . . . . 57 
3.3 .6 Steps duration $\ldots \ldots \ldots \ldots \ldots$

3.3 .7 Step length estimation . . . . . . . . . . . . . . . 58

3.3.8 Walking speed and variability parameters . . . . . . . . . . 59

3.4 Evaluation method . . . . . . . . . . . . . . . 60

3.5 Risk Profile . . . . . . . . . . . . . . . . . . . . . 62

4 Results and Discussion $\quad 67$

4.1 Inertial sensors signals . . . . . . . . . . . . . . . . 67

4.2 Events detection . . . . . . . . . . . . . . . . . . . 70

4.3 Integration . . . . . . . . . . . . . . . . 75

4.4 Step length, duration and velocity evaluation . . . . . . . . . . 76

4.5 General discussion of results . . . . . . . . . . . . . . . . . . 79

5 Conclusions and future Work $\quad \mathbf{8 1}$

5.1 Future work . . . . . . . . . . . . . . . . . 82

$\begin{array}{ll}\text { References } & 85\end{array}$

A Screening tools $\quad 95$

A.1 Single Task Performance Measures . . . . . . . . . . . . . . . . . . . 95

A.1.1 One-legged stand (OLS) . . . . . . . . . . . . . . . . 95

A.1.2 Functional Reach Test (FR) _ . . . . . . . . . . . . . . . 95

A.1.3 Sit-to-stand transfer with 5 repetitions (STS5) . . . . . . . . . . 95

A.1.4 Rapid Step Test (RST) . . . . . . . . . . . . . . . . . . . . 95

A.1.5 Alternative Step Test (AST) . . . . . . . . . . . . . . . 96

A.1.6 Four square step test (FSST) . . . . . . . . . . . . . . 96

A.1.7 Maximal Step Length (MSL) . . . . . . . . . . . . . . . . 98

A.1.8 Comfortable Gait Speed (CGS) . . . . . . . . . . . . . . . . 98

A.1.9 Timed Up \& Go Test (TUGT) . . . . . . . . . . . . . . . . . . 98

A.1.10 Romberg's Test . . . . . . . . . . . . . . . . . . . . . . . . 99

A.1.11 Near-tandem standing balance (NTSB) . . . . . . . . . . . . . 99

A.1.12 Turn $180^{\circ} \ldots \ldots \ldots \ldots$. . . . . . . . . . . . . . . 99

A.2 Multi-task Measures . . . . . . . . . . . . . . . . . . . . . . 99

A.2.1 Performance-Oriented Mobility Assessment (POMA) . . . . . . . 99

A.2.2 Berg Balance Scale (BBS) . . . . . . . . . . . . . . 100

A.2.3 Dynamic Gait Index (DGI) . . . . . . . . . . . . . . . . 101

A.2.4 Balance Evaluation Systems Test (BESTest) . . . . . . . . . . . . 101

A.2.5 Physiological Profile Assessment (PPA) . . . . . . . . . . . . . 101

A.3 Self-reported tools . . . . . . . . . . . . . . . . . . . 103

A.3.1 The Falls Risk for Older People in the Community scale (FROP-

Com) . . . . . . . . . . . . . . . . 103

A.3.2 Activities-Specific Balance Confidence Scale (ABCS) . . . . . . 103

A.3.3 Falls Efficacy Scale (FES) . . . . . . . . . . . . . . . . . . . 104

A.3.4 Modified Falls Efficacy Scale (MFES) . . . . . . . . . . . . . . . 104

A.3.5 Functional Status Questionnaire (FSQ) . . . . . . . . . . . . 105

A.3.6 The Barthel Index (BI) . . . . . . . . . . . . . . . . . 106

A.3.7 Katz Index of Activities of Daily Living (Katz ADL) . . . . . . 108

A.3.8 Lawton Instrumental Activities of Daily Living (Lawton IADL) . 109 


\section{CONTENTS}

A.3.9 Health Status Questionnaire (HSQ) . . . . . . . . . . . . . . . 109

A.3.10 Self report version of the HOME-FAST (HOME FAST-SR) . . . 109 
CONTENTS 


\section{List of Figures}

2.1 Aetiology model for falls . . . . . . . . . . . . . . . . . 7

2.2 Age-adjusted nonfatal fall injury rates . . . . . . . . . . . . 8

2.3 Risk factors interaction . . . . . . . . . . . . . . . . . 10

2.4 Occurrence of falls $v s$. number of risk factors . . . . . . . . . . . 11

2.5 Components for successful balance control . . . . . . . . . . . . . 13

2.6 Assessment and Management of falls . . . . . . . . . . . . . . . . . . . 16

2.7 AGS/BGS Clinical Practice Guideline . . . . . . . . . . . . . . . . . . 18

2.8 Screening and Assessment of fall risk . . . . . . . . . . . . . . . . . . 19

2.9 Walkway with one force-plate . . . . . . . . . . . . . . . . 21

2.10 Codamotion 3D measurement instrument . . . . . . . . . . . . . . 22

2.11 Implementation of the TUGT using inertial sensors . . . . . . . . . . 27

2.12 Example of a left and right shank angular velocity signals . . . . . . . . . . 28

2.13 Example of accelerometer data during a DR . . . . . . . . . . . . . . 29

2.14 SiSt and StSi transitions in a young male vs. an elderly female . . . . . . 30

2.15 SiSt transition . . . . . . . . . . . . . . . . . . 30

2.16 Acceleration signals during SiSt transition . . . . . . . . . . . . . . 31

2.17 Acceleration signals during StSi transition . . . . . . . . . . . . . 31

2.18 Gait cycle . . . . . . . . . . . . . . . . . . 32

2.19 Antero-posterior acceleration signal at the level of pelvis . . . . . . . . . . 34

2.20 Medio-lateral acceleration signal at the level of pelvis . . . . . . . . . . . . . 34

2.21 Vertical acceleration signal at the level of pelvis . . . . . . . . . . . . . . 35

2.22 Pelvis and head acceleration signals . . . . . . . . . . . . . 36

2.23 Lower trunk position of an inertial sensor . . . . . . . . . . . . . 36

2.24 Decision Tree . . . . . . . . . . . . . . . . . . . . 38

2.25 Model of the vertical displacement of the pelvis during walking . . . . . 39

2.26 Block Diagram . . . . . . . . . . . . . . . . . . . 43

2.27 Acceleration sensor coordinate system . . . . . . . . . . . . 45

3.1 Phone coordinate system and ISB conventions . . . . . . . . . . . 53

3.2 Zero Crossings and maximums detection . . . . . . . . . . . . . . 54

3.3 Sliding standard deviation illustration . . . . . . . . . . . . . 55

3.4 Illustration of a toe off detection . . . . . . . . . . . . . . 57

3.5 Gait phases from right and left side. . . . . . . . . . . . . . . 57

3.6 Sitting height . . . . . . . . . . . . . . . . . . 60

3.7 Tests scenario . . . . . . . . . . . . . . . . . 61

3.8 Illustration of step length estimation using video recording . . . . . . . 62

3.9 Part of FROP-Com assessing history of falling . . . . . . . . . . . . . . 64 


\section{LIST OF FIGURES}

3.10 Part of FROP-Com assessing medications . . . . . . . . . . . . . 65

3.11 Part of FROP-Com assessing medical conditions . . . . . . . . . . 66

4.1 Inertial sensors signals obtained from phone sensors. . . . . . . . . . . . 68

4.2 Example of a bad forward acceleration signal . . . . . . . . . . . . . 69

4.3 Illustration of acceleration errors due to wrong phone's orientation . . . . 70

4.4 Zero Crossings detection in a real signal . . . . . . . . . . . . . . . 71

4.5 Maximums maintained after eliminating those due to noise . . . . . . . 71

4.6 Acceleration and Standard deviation signals . . . . . . . . . . . . . . 72

4.7 Retained maximums after eliminating those on the standing phase . . . . 72

4.8 Foot contacts detection . . . . . . . . . . . . . . . . 73

4.9 Examples of bad foot contacts detection. . . . . . . . . . . . . 73

4.10 Forward acceleration and gravity . . . . . . . . . . . . . . . . 74

4.11 Toe offs and heels strikes on vertical linear acceleration . . . . . . . . . 75

4.12 Double integration of vertical acceleration. . . . . . . . . . . . . 76

4.13 Expected and Measured mean step length . . . . . . . . . . . . . 77

4.14 Expected and Measured mean step duration . . . . . . . . . . . . . 77

4.15 Expected and Measured velocity . . . . . . . . . . . . . . . . 78

A.1 Functional Reach Test . . . . . . . . . . . . . . . . . . . 96

A.2 Illustration of the STS5 execution . . . . . . . . . . . . 96

A.3 Illustration of the AST execution . . . . . . . . . . . . . . . . 97

A.4 An example of a FSST setup . . . . . . . . . . . . . . . . . . 97

A.5 Illustration of the TUGT . . . . . . . . . . . . . . . . . . . . 98

A.6 Performance-Oriented Mobility Assessment . . . . . . . . . . . . . . 100

A.7 The Berg Balance Test Items . . . . . . . . . . . . . . . . . . . . . 101

A.8 The six systems/sections evaluated on BESTest . . . . . . . . . . . . 102

A.9 Summary of tests performed under each system category of BESTest . . . 102

A.10 PPA short-form tests . . . . . . . . . . . . . . . . . . . . 103

A.11 Activities-Specific Balance Confidence Scale (ABCS) . . . . . . . . . . . 104

A.12 Falls Efficacy Scale (FES) . . . . . . . . . . . . . . . . . . . 105

A.13 Modified Falls Efficacy Scale (MFES) . . . . . . . . . . . . . . . 106

A.14 Functional Status Questionnaire (FSQ) . . . . . . . . . . . . . . . . 107

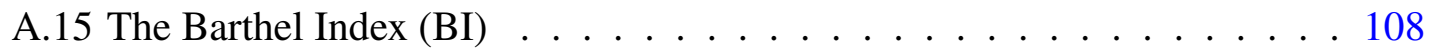

A.16 Katz ADL . . . . . . . . . . . . . . . . . . . . . . . 109

A.17 Lawton Instrumental Activities of Daily Living (Lawton IADL) . . . . 110

A.18 Health Status Questionnaire (HSQ) . . . . . . . . . . . . . . . . 111 


\section{List of Tables}

3.1 Self reported tools and risk factors. . . . . . . . . . . . 63

3.2 Risk profile. . . . . . . . . . . . . . . . . 66

A.1 FSQ Warning Zones . . . . . . . . . . . . . . . . . . . . . 106 
LIST OF TABLES 


\section{Abbreviations}

$\begin{array}{ll}\text { ABC } & \text { Activities-Specific Balance Confidence } \\ \text { ADL } & \text { Activities of Daily Living } \\ \text { ADT } & \text { Android Developer Tool } \\ \text { AGS } & \text { American Geriatrics Society } \\ \text { API } & \text { Application Programming Interface } \\ \text { AST } & \text { Alternative Step Test } \\ \text { BBS } & \text { Berg Balance Scale } \\ \text { BESTest } & \text { Balance Evaluation Systems Test } \\ \text { BGS } & \text { British Geriatrics Society } \\ \text { BI } & \text { Barthel Index } \\ \text { BSN } & \text { Body Sensor Networks } \\ \text { CGS } & \text { Comfortable Gait Speed } \\ \text { COP } & \text { Centre of pressure } \\ \text { COM } & \text { Centre of mass } \\ \text { CV } & \text { Coefficient of Variation } \\ \text { DDMS } & \text { Dalvik Debug Monitor Server } \\ \text { DGI } & \text { Dynamic Gait Index } \\ \text { FES } & \text { Falls Efficacy Scale } \\ \text { FFT } & \text { Fast Fourier Transform } \\ \text { FIR } & \text { Finite Impulse Response } \\ \text { FRT } & \text { Functional Reach Test } \\ \text { FRAT } & \text { Fall Risk Assessment Tool } \\ \text { FROP-Com } & \text { The Falls Risk for Older People in the Community scale } \\ \text { FSQ } & \text { Functional Status Questionnaire } \\ \text { FSST } & \text { Four Square Step Test } \\ \text { HOME FAST } & \text { Home Falls and Accidents Screening Tool } \\ \text { HSQ } & \text { Health Status Questionnaire } \\ \text { IADL } & \text { Instrumental Activities of Daily Living } \\ \text { ICD } & \text { International Classification of Diseases } \\ \text { ICECI } & \text { International Classification of External Causes of Injury } \\ \text { IIR } & \text { Infinite Impulse Response } \\ \text { ISB } & \text { International Society of Biomechanics } \\ \text { JDK } & \text { Java Development Kit } \\ \text { MFES } & \text { Modified Falls Efficacy Scale } \\ & \end{array}$




\section{ABBREVIATIONS}

MFS Morse Fall Scale

MSL Maximal Step Length

NICE National Institute for Clinical Excellence

NTSB Near-tandem standing balance

OLS One-legged stand

POMA Performance-Oriented Mobility Assessment

PPA Physiological Profile Assessment

RST Rapid Step Test

SD Standard Deviation

SDK Software development kit

SiSt Sit-to-Stand

STRATIFY St Thomas' Risk Assessment Tool

StSi Stand-to-Sit

STS5 Sit-to-stand transfer with 5 repetitions

TGBA Tinetti Gait and Balance Assessment

TUGT Timed Up \& Go Test

WHO World Health Organization 


\section{Chapter 1}

\section{Introduction}

The progressive ageing of population and the increasing number of people living to an advanced age creates new social and economic challenges, concerning people's health and well-being. In particular, falling is a serious and common problem facing people aged 65 years or older [1].

Due to age-related changes and frailty, falling on the elderly can lead to serious physical and psychological consequences, such as injury, suffering, fear, depression, loss of independence, reduced quality of life and even death. More than an individual problem, falling can also be problematic for family and carers, having even an economic impact $[1,2,3,4,5]$.

With the growing number of older people in the population, these problems can be further marked in a near future $[3,6]$. This should be further problematic for older people living in the community where help or medical assistance should be more difficult to accomplish [7].

In order to reduce the consequences of a fall, and to give faster assistance when a fall occurs, several products are now commercially available, e.g. the fall detection systems $[7,8]$. These are essentially reactive, and don't prevent the occurrence of falls and some of their related consequences.

Since the last 25 years, several studies have described the falls aetiology, changing the idea that falling was unpredictable and not preventable [5]. Several tools and questionnaires are now available to determine and reduce the fall risk and assess specific risk factors for falling [6]. Prevention strategies imply interventions on potentially modifiable risk factors, such as medications or hazards at home [9].

Although the effectiveness of such interventions is recognized, fall prevention is not yet widely practised in geriatric clinics, making falling an undertreated public health issue 
[5]. It is now recognized that earlier detections of risks are necessary in order to earlier apply prevention strategies [6,9].

Recently, some systems using wearable devices with motion sensors, such as accelerometers and gyroscopes, have been developed to evaluate functional ability and mobility during the performance of some daily movements/tasks. In contrast to clinical tests, these methods are self-administrable and can be used outside clinical environments, being able to anticipate the detection of problems associated with increased risks of falling, and therefore to administer/modify prevention strategies at an earlier stage $[6,10,11]$.

On this project, mobile phone is suggested as a potential instrument for fall risk screening/assessment, in order to improve the falls risk management. They have the advantage of being portable and are equipped with sensors, e.g. accelerometer, that can be used to evaluate several movements.

Phone would enable unsupervised assessments of falls risk, so that a more frequent monitoring of risks could be achieved and earlier administration of prevention strategies can be possible. Additionally, history of risk factors can be done over time, so that more information regarding fall prevention strategies can be accessible to the doctor. The tracking of falls risk over time also provide the feedback necessary for modification of ineffective interventions, which cannot be accomplished by recent strategies [6].

This report is under the scope of Dissertation project, necessary for granting of the degree of Master in Bioengineering. The project was developed at Fraunhofer AICOS Portugal.

\subsection{The problem}

Falling is a serious concern affecting older people. If their occurrence is not prevented in a near future, and with the increasing proportion of older people in population, the problem will become increasingly serious. Therefore, falls prevention is becoming a challenge and it should be of great importance that more actions are done in order to prevent falls [1].

Currently, some tools exist for falls risk screening, which is performed in a clinical setting. These clinical-centric models are only available to those who live near a falls clinic and are at high risk of falling, and frequently they are costly, time-consuming and require expert knowledge. This model of care is then becoming increasingly unsatisfactory [11].

A proactive community-based strategy will be therefore required, so that early detection of higher risks of falling can be done [11].

Some sensor-based systems already exist to evaluate some mobility performances. Although these systems are portable and not expensive, the use of such a system poses restrictions on its use, since an extra device needs to be worn, which frequently is not aesthetic. 


\subsection{Motivation and Objectives}

Current research indicate that portable systems using inertial sensors are equivalent or better than the existing scales to measure risk of falling. They have the advantage of being portable, low-cost and easy-to-use, being ideal for assessing fall risk in community setting [11].

These kind of sensors are available on modern mobile phones. These daily-used devices can then be used to perform similar evaluations, so that the individual's risk of falling can be determined. Phone can also be used to measure several risk factors, using the same questionnaires currently used by doctors, so that a risk profile can be built over time, providing new insights about fall prevention strategies.

When compared with the expensive equipments that exist in falls clinics, the phonebased solution would be cheaper, more accessible and, due to its portability, would enable a more frequent assessment of fall risk, earlier alerting the older person and doctors for an increased risk of falling.

The main objective of this project is then to study some possible ways to adapt the mobile phone for fall risk screening purposes. This should be done based on the clinically validated questionnaires and tests and the most recent studies on fall risk prediction. The target device will be an Android based smartphone, equipped with inertial sensors.

The developed system should be adapted to the target users (older persons), i.e. considering that it should be easy-to-use, safe and user friendly. A result should be provided, so that the individual can recognize a high or low risk, and a history of results over time should be built.

For the valuable use of technology, it must be accurate, reliable and repeatable [11, 12]. The evaluation of the developed application is then required, using a small user group.

\subsection{Benefits}

To my best knowledge, currently, no smartphone application exist to assess the risk of falling of an individual. This project could then be an innovation, that would provide new ways for unsupervised, low-cost, early and frequent predictions of fall risk, so that proper interventions on risk factors for falling can be done before the occurrence of a fall. Earlier detections of risks would enable earlier application of prevention strategies, that are proved to be effective in preventing the occurrence of falls.

An enormous advantage of using the phone would be the more frequent assessment of risks, which would be associated with time, providing new insights about risks evolution and falling events. This would be useful to the doctor, who could design better prevention 
strategies and also modify the ineffective ones, considering the evolution of risks affecting the older person.

This should be of great value, not only for health care systems, but also to older people, who can benefit from less suffering if falls and fall-related injuries can be avoided. This is also important for family and carers, who would have less responsibilities, concerns and costs.

Latter, an improvement of independence and quality of life with fewer restriction of activities should be observed. All the consequences related with the occurrence of falls should also be reduced.

A better management of falls would then be expected, so that falling can no longer be a public health and economic problem, even with the increasing proportion of older people in society.

\subsection{Report's structure}

Apart from Introduction (Chapter 1), this report consists of four chapters. First, a literature review with some background concepts is provided (Chapter 2), so that falling and fall management can be more deeply understood and gaps on the existing strategies can be identified. Using the background knowledge, a method was developed and described in detail in Chapter 3. Results obtained using these methods are then presented and discussed, with the identification of succeeds and the necessary improvements (Chapter 4). At least, on Chapter 5, the conclusions related with this project are presented and some future work to potentially improve it is also identified. 


\section{Chapter 2}

\section{Background and Literature Review}

In this chapter a literature review on fall risk prediction and some fundamental background concepts are presented. The information is provided having in consideration the project's description and aims, so that a final solution can be presented and justified based on a sequential literature review.

\subsection{The process of ageing}

The advances in medical technology, health care, nutrition and sanitation are resulting in lower mortality rates throughout the world, so that the oldest segment of population is growing faster [13].

The concept of "old age" is commonly defined by the chronological date, that is, the whole years since the day of birth. In absolute terms, an older person is considered to be someone aged 65 years and above [1,13]. This limit is only based on economic criteria, since in many countries this is the normal retirement age [13].

Ageing is determined by morphological and physiological wear of organs and systems. Old people have a lower capacity to cope with diseases and also to adapt to environmental changes. They get tired more easily, are slower, and have less mobility and agility. They have worse perceptive skills, mental agility and memory. Although a decline in several biological systems has been documented, the cause of ageing is not yet agreed upon [9, 13].

Heredity, environment and lifestyle factors are however recognized to influence the ageing process, which makes old people diverse in their medical, psychological and physical status. As a consequence, two people may be of the same age, but differ in their status and capacities, since each individual has a unique ageing rate. Then, chronological age is not perhaps the best choice when describing and older person [13]. 
The way people age can be categorized as usual (or normal), pathological (or abnormal) and successful ageing. The usual ageing is characterized by a gradual decline in body function and refers to the way most people age, leading to physical impairments, disease, functional limitations, and eventually the onset of disability and death. The pathological ageing refers to the premature disability and death, as a consequence of genetically predispose to some diseases or high-risk lifestyle. The successful ageing, on the other hand, refers to better physiological and psychosocial characteristics than the average in the later life. However, some decline and functional loss are inevitable in the very old age [13].

\subsection{Falls}

Old age is frequently associated with an increased risk of falling and fall-related injuries.

Falls are not an inevitable result of ageing, but with the increasing number of older people in population, the concerns related with falling are becoming more common and serious, being increasingly important to act in order to prevent injuries [1].

\subsubsection{Definitions}

Different fall definitions are used on the literature, i.e. in relation to the cause of the fall [2].

In 1987, the Kellogg International Group on the Prevention of Falls defined a fall as an "unintentionally coming to the ground or some lower level and other than as a consequence of sustaining a violent blow, loss of consciousness, sudden onset of paralysis as in stroke or an epileptic seizure" [14].

In 1989, M. Nevitt classified as "syncopal falls" the falls occurring due to loss of consciousness (syncope or seizure) or paralysis [15].

The International Classification of External Causes of Injury (ICECI), which is related to the World Health Organization (WHO) International Classification of Diseases (ICD), provides ways to classify the external causes of injuries. According to ICECI, a fall is a "nonsyncopal event not attributable to sustaining a violent blow, loss of consciousness, stroke or epileptic seizure", which includes falling on same level or falling from a height [16].

According to National Institute for Clinical Excellence (NICE) guidelines a fall is defined as an event whereby an individual comes to rest on the ground or another lower level with or without loss of consciousness [1].

A proper definition of falling is important in order to make reliable comparisons between fall risk assessment tools, whose accuracy may vary in relation to the number of people considered to have fallen, which also affects the incidence of falling. The tendency 
is to include the syncopal falls in studies of older people living in institutions, and exclude them from the studies of those living in the community [2, 17].

In relation to the person who falls, there are also different applicable definitions. A faller is usually defined as someone who has fallen at least once over a set period of time, usually six or twelve months. A recurrent faller, in turn, is someone who has fallen twice or more during the time period. However, some authors identify the faller as someone who has had at least two falls and a recurrent faller as someone who has experienced three or more falls over a period of time. This last definition is based on findings which assume that the "once only faller" has characteristics more closely related to non-fallers than to "twice or more fallers". One fall can be a coincidence, but falls in the recurrent faller seems more like a pattern [2].

In this project, the Kellogg's definition of fall was adopted and a recurrent faller was considered someone who has fallen twice or more during a time period.

\subsubsection{Aetiology}

Physically, a fall occurs when the person's centre of gravity moves outside his/her base of support and insufficient, ineffective or no effort is made to restore balance [5].

It is recognized that falls have a multi-factorial aetiology, including biological, behavioural, environmental and socioeconomic factors [3]. Their interaction (illustrated on Figure 2.1) may vary from individual to individual and may be a cause of falling [4].

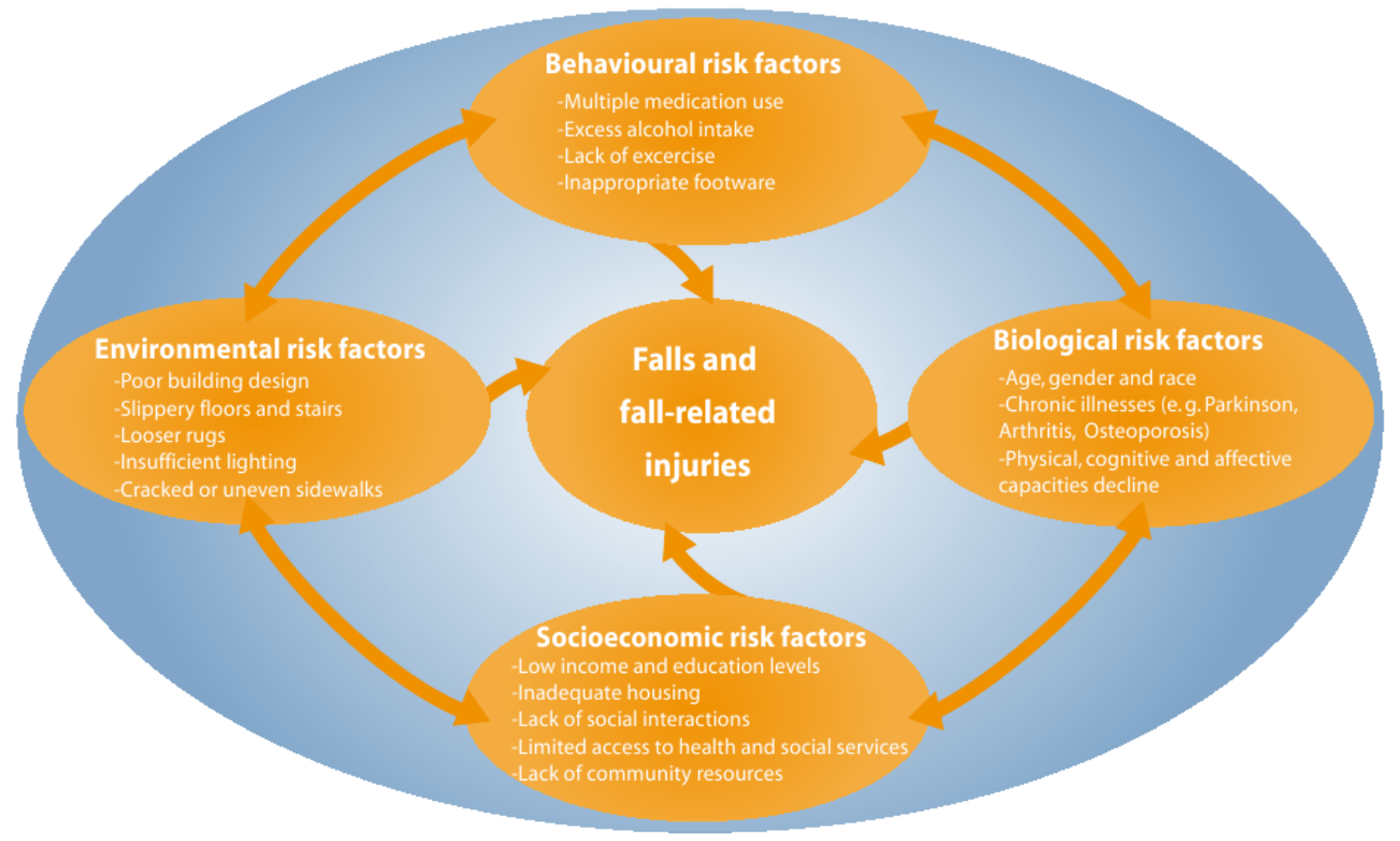

Figure 2.1: Aetiology model for falls. Source: [3]. 
The biological factors include the decline in physical, cognitive and affective capacities of an individual, which in addition to extrinsic factors such as environmental hazards, can result in a fall. Human actions, emotions and daily choices (e.g. the intake of multiple medications or the sedentary behaviour) can also contribute to the occurrence of a fall [3]. Either the postural responses to challenges posed by the environment either the ones posed by movements such as transfers or walking can be a cause of fall [5]. Other factors, such as social condition and economic status, including low education levels, inadequate housing, lack of social interaction or the limited access to health and social care can also contribute to higher risks of falling [3].

\subsubsection{Incidence}

Falls are among the most common and serious cause of accident facing persons aged 65 or over [17].

As the proportion of older people in the population is growing, more people are being prone to fall. An estimated 28 to $35 \%$ of people aged 65 years and older fall each year, and after the age of 70 years the rates are higher (32-42\%) [3].

The frequency of falls increases with age and until the age of 75, woman fall more often than men (see Figure 2.2). This is due to increased impairment in mobility and to a higher number of associated risk factors among woman. After the age of 75, the frequency is similar for both woman and men [13]. Also, the falls exponentially increase with age-related biological changes [3].

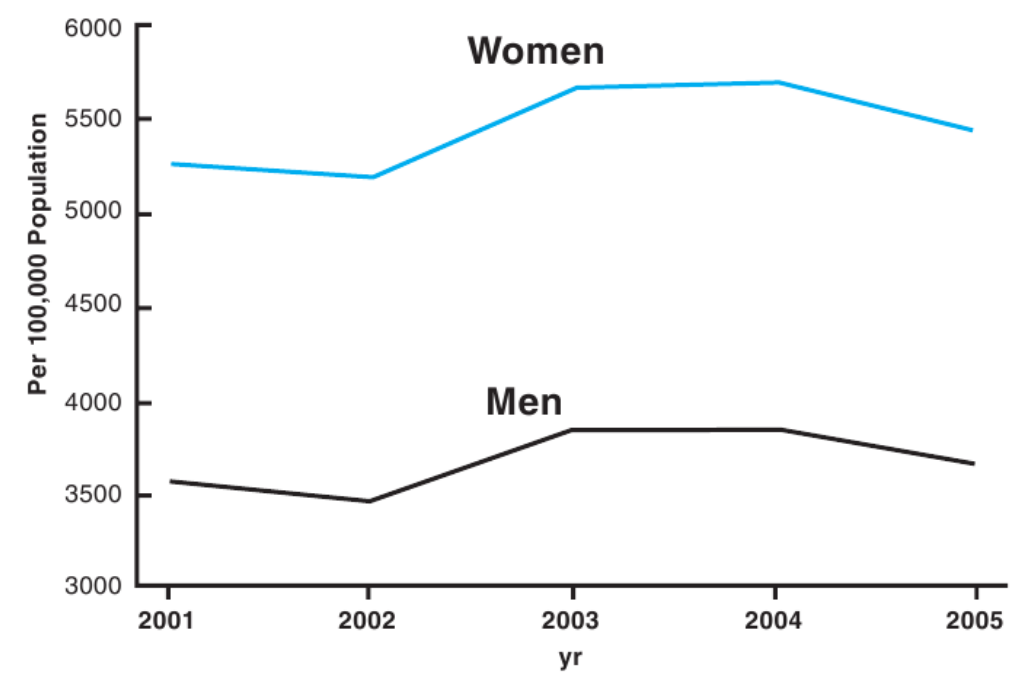

Figure 2.2: Age-adjusted nonfatal fall injury rates among woman and men aged 65 years and older in United States. Rates are per 100000 people and the age-adjusted to the 2000 U.S. population. Source: [5]. 


\subsubsection{Impact}

Falling has an impact on quality of life, health and health care costs. With the augment of life expectancy, new demands for healthcare and services will arise in a near future. In fact, it is expected that if any preventive measures are taken, the amount of injuries caused by falls will be $100 \%$ higher in 2030 [1, 2, 3].

Although falls in older people typically consists of relatively low impact energies caused by falling from the stairs or the standing position, the consequences of such a fall can still be serious. Due to age-related changes, elderly persons are more frail and then more susceptible to injuries than younger people $[1,4]$.

An injurious fall refers to a fall resulting in a fracture or soft tissue damage that requires treatment. Fractures (e.g. hip, pelvis, femur, hand and ankle fractures), head trauma, lacerations, upper limb injuries and spinal cord injury are some examples of such an injury. International rates of injurious falls range from 0.55 to 1.00 per person-year $[1,4,5]$.

Hip fractures are probably the most feared fall-related injury. After such an event, approximately half of older persons cannot return home or live independently, and $20 \%$ die within a year of fracture $[3,5] .40 \%$ of all injury deaths is due to falls [3].

Falling is associated with increased morbidity, reduced functioning, loss of independence and premature hospital and geriatric admission, which leads to restriction in daily activities [3, 13].

Psychological consequences can also arise from falling, e.g. loss of confidence and fear of falling again. This leads to an increased risk of inactivity and to a reduction in the ability to perform activities of the daily living, even if the fall didn't result in a serious injury. After all, the quality of life and social interaction can be compromised, which can also be associated with depression [1, 17].

Both the incidence of falls and the complications associated with falls increase considerably after the age of 60 . They account for $4 \%$ of all hospital admissions and they are also a major reason for admission to a residential care setting, even when no serious injury has occurred $[4,13]$.

The economic impact of falling is critical to family, community and society. Costs are related to treatments and rehabilitations, adequate services and losses on productivity for individuals and carers. The costs related to hospital and nursing home care are greater in people who fall when compared to matched non-fallers $[3,4]$.

\subsection{Risk factors for falls}

The prevention of falling implies an early intervention on key risk factors that identify people at high risk of a first or subsequent fall, thus with a higher probability of injury or 
other related consequences. In the literature, it can be identified a large number of risk factors, illustrating the complex causality of falls.

Different study designs are used to identify risk factors for falling. The prospective cohort study design has been identified as the strongest for searching evidence for risk factors. Other designs, e.g. retrospective cohort studies, are more susceptible to confounding and biases, because older people can have difficulties to recall past falls $[1,2,9]$. Frequently, the studies are focused on people aged 65 years and over that live in a specific kind of residence, such as the community-dwelling residents (i.e. those living in ordinary housing) and those living in institutions, e.g. hospital settings, nursing homes and residential care facilities [9].

Many fall risk factors are common to those living in community and institutions. However, the prevalence and modifiability of risk factors can greatly differ between the two settings [18].

Risk factors for falling can generally be categorised into intrinsic, or patient-related, behavioural and environmental factors (Figure 2.3). The first ones include, for example, advanced age, chronic diseases, muscle weakness and gait or balance disorders. Behavioural factors are related to risks-taking, such as medications intake, alcohol consumption or hazardous activities. The environmental risks can frequently be encountered inside or outside the home, e.g. obstacles on the floor or poor light [19].

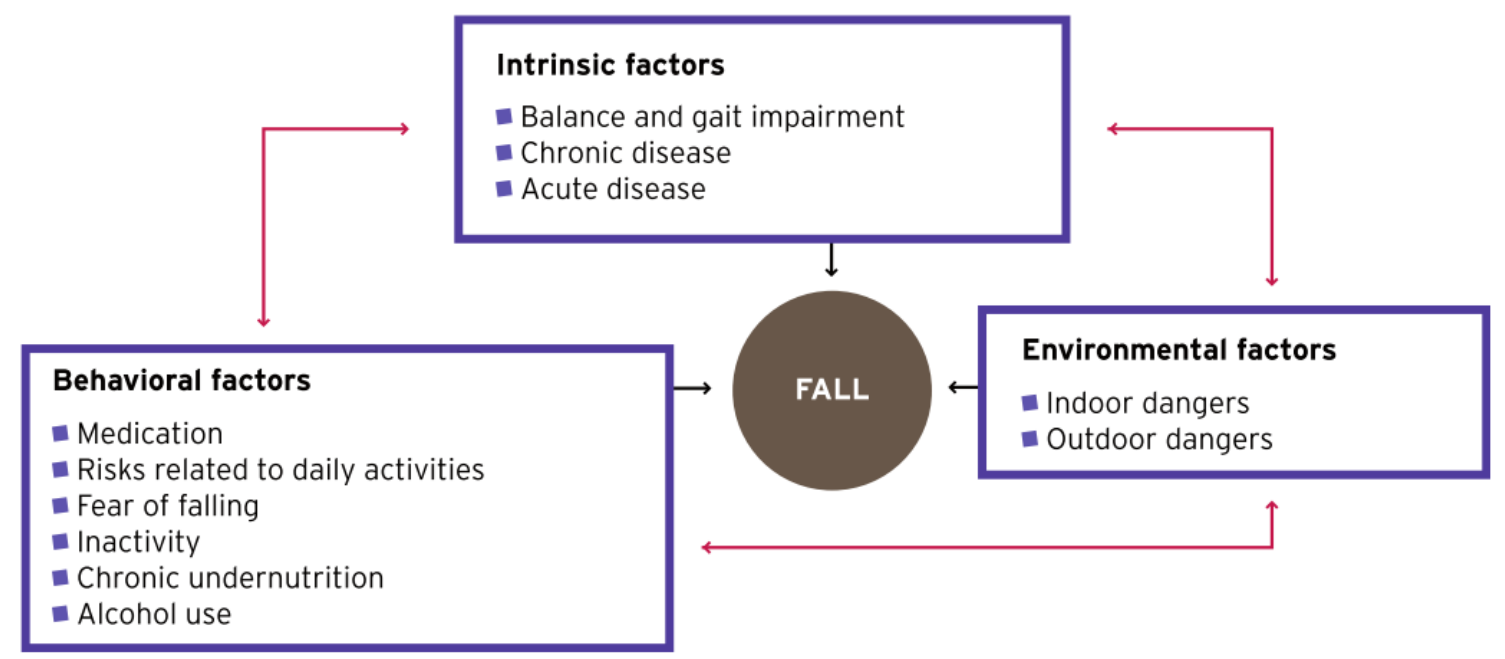

Figure 2.3: Risk factors interaction. Source: [19].

The risk factors intervene on the mechanisms of fall and interact [19]. A probable synergism between multiple risk factors is also recognized [20]. As can be seen in Figure 2.4, the risk of falling increases with the number of risk factors affecting the person [5].

Falling can be classified as a recurrent condition, because over time it can cause its own risk factors, e.g. injury and fear of falling [18]. 


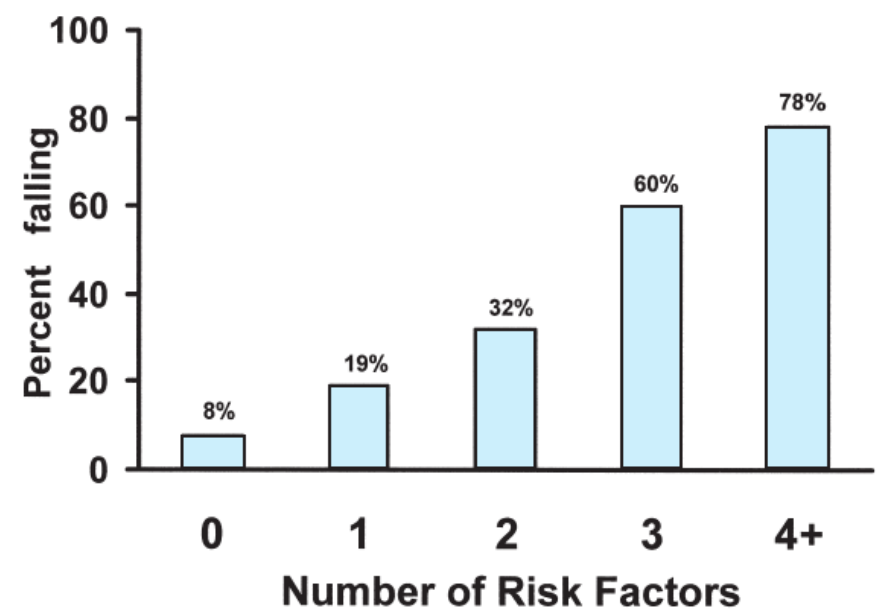

Figure 2.4: Occurrence of falls increases with the number of risk factors. The study was performed with 336 persons older than 75 years living in the community. The risk factors include: sedative use, cognitive impairment, low-extremity disability, palmomental reflex, foot problems and balance/gait abnormalities. Source: [5].

The number of intrinsic risk factors affecting the older persons affects the frequency of some extrinsic or behavioural factors. For example, as a response to a disease, multiple medications can be prescribed. Consequently, the environmental factors that usually were safe, e.g. stairs, can suddenly become environmental hazards [18].

On the following paragraphs, the most important risks factors for falling will be explained regarding their interaction and association with increased risk of falling.

\section{Falls history}

Falls history has a high evidence of being associated with an increased risk of falling and is a significant predictor of falling, according with current recommendations [1, 19].

The previous falls are often referred as the gold standard when assessing fall risk, because they are related with lower levels of physical functioning, being therefore a marker of frailty [9].

\section{Advanced age}

As described on Section 2.1, old age is associated with morphological and physiological wear of organs and systems $[9,13]$, including changes on the neurological, musculoskeletal and sensory systems.

Old people do not adapt their sensory inputs (i.e. visual, vestibular and proprioceptive inputs) to changing environments as well as younger do, have slower reactions time and a reduced muscle strength $[5,9,21]$. 
In general, these changes affect the control of postural stability and balance and the ability to reorganize balance reactions when an obstacle is present [21]. The relation between balance and risk is explained on the next paragraphs.

\section{Mobility, balance and gait}

Balance is a generic term to describe the dynamics of body posture that requires a motor control of gravitational forces to maintain posture and a control of acceleration forces to maintain equilibrium. Results from several studies suggest that the ability to maintain balance is critical to avoid a fall $[9,21]$.

With ageing, or due to the occurrence of diseases, there are several changes in musculoskeletal, neurological and sensory systems, which are associated with a reduced capacity to control balance and gait during daily activities [5].

The musculoskeletal changes comprises a reduced muscle strength, which is related with a decreased muscle mass and fibres, loss of motor units and decreased maximal force production. Muscle mass and strength decline with age, or as a consequence of diseases and inactivity. This implies that a less capacity exists to deal with a balance disturbance, which can lead to a fall $[5,9,21]$.

Changes occurring in sensory inputs can alter the control of postural stability. Particularly, the difficulty to readjust sensory inputs under changing environments can increase the risk of falling $[5,9,21]$.

The changes that occur in the nervous system, e.g. decreased number of neurons and decreased conduction velocity, lead to a slower information processing, which results in a slow reaction time in order to avoid a fall and re-establish balance [5, 9, 21].

The components implied in a successful balance control are illustrated in Figure 2.5.

Maintaining balance while walking can be an even greater challenge for older people. Walking is a mechanically complex task that requires a changing base of support, i.e. transferring balance from one foot to the other, which requires a regulation of the centre of the mass (COM). During walking, it is the visual information that enables the identification of upcoming disturbances (e.g. obstacles, uneven surfaces), aiding to establish the accurate location of the next centre of mass position. If some unexpected perturbation occurs, an attempt to re-establish the balance is done, which requires a fast corrective reaction [21].

A successful mobility, i.e. the ability to independently and safely move oneself from one place to another, requires an appropriate balance control [9].

A lower capacity to control balance and gait is responsible to accelerate with a sedentary life, which causes a reduced functional capacity in basic daily activities. This can lead to impaired balance and gait, and consequently, an increased risk of falling in everyday activities [9]. 


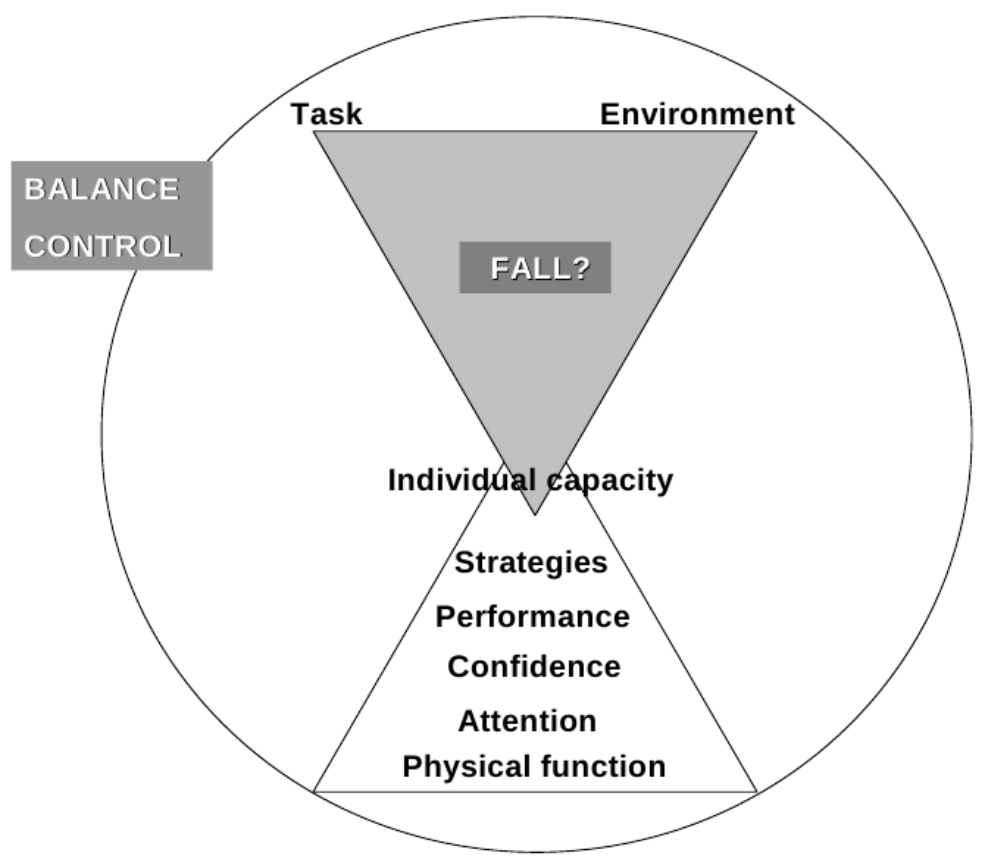

Figure 2.5: Important components for successful balance control. It is the relationship between individual capacity, the task undertaken and the environment in which the activity is performed that will affect the outcome and subsequent fall risk. Source: [9].

Considering the centrality of balance in a fall event, some authors support the idea that risk factors can interact through causal pathways with the balance at their core, i.e. they can mechanistically cause falls via balance and gait systems. This means that the causal role of each risk factor can be identified with reference to its effects on balance [18].

\section{Fear of falling}

Fear of falling refers to the lack of self-confidence in performing normal activities without falling [22], and is a significant predictor of future falling [1].

It can either be a post-fall syndrome (i.e. a consequence of falling) or an anticipatory anxiety occurring in those who have not fallen. The latter is called ptophobia, and leads to a phobic reaction to standing or walking $[1,4]$.

The loss of confidence in balance ability or low confidence at avoiding falls can result in a fear. This leads to an increased risk of inability, decreased mobility, gait and balance abnormalities, use of walking aids, a reduction in the capacity to perform activities of the daily living and reduced quality of life. Fear of falling is also associated with anxiety and depressive symptoms $[1,4,22]$.

The loss of self-confidence can result in self-imposed functional limitations [20] The restriction of activities can be a useful adaptation to physical limitations, but can also be 
an unnecessary avoidance of activities that the person is capable of doing. The adaptation of older people's gait, i.e. a slower gait velocity, is often described as a more cautious gait when fear of falling is present [22].

\section{Medications}

Older persons often take more than four medications each day, due to multiple diseases. Several studies show that the use of four or more medications, either prescribed or no prescribed, increases the risk of falling. This is due to the expected effect of drugs, for example, on cognitive functioning, fluid and electrolyte balance or blood pressure [5].

Hendrich et al. (1995) also refers that the side effects of medications, for example, sedation, hypotension, impaired balance, altered elimination and impaired reaction time must be more predictable of falling than the medication itself [23].

A review of evidence regarding medications and falls concluded that psychotropic drugs, e.g. antidepressants, sedative-hypnotics and antipsychotic medications, were commonly implicated in falls [24]. The more medications of any type a person takes, the higher the risk of falls [25].

\section{Environmental hazards}

As referred earlier, environment has a great impact on the risk of falling. When a declined balance and mobility is present, the individual is more susceptible to falls, since they have reduced capacity to adapt and react to environmental changes [5, 9, 21].

Some studies have examined the threats implicated in falls and check-lists were created to review and improve home safety [5]. Items include, for example, slippery floors, unsafe paths around the house, no indoor step rails, poor lighting, unclear stair edges and hazardous care of pets [26].

\section{Hazardous activities}

The behaviour of the individual has a great impact on the risk of falling, since older persons with impaired balance and strength can do activities that are beyond their capabilities.

Frequently, this is due to poor judgement, the desire to maintain independence or the lack of help from family and friends [5].

\section{Diseases}

Chronic and acute diseases increase the risk of falling in the older people. The simultaneous presence of two or more diseases can further increase this risk [19]. 
Indeed, the frequency of diseases is higher among older persons and can cause several changes in musculoskeletal, neurological and sensory systems [19]. As already explained, these systems are implicated in the capacity to control balance and gait during daily activities, so that any problem in these systems can lead to an increased risk of falling [5, 9, 21].

Frequently, some drugs are used to cope with diseases, which can also be a source of increased risk [19].

\subsection{Principles of practice}

Currently, some guidelines and recommendations exist for the management of falls in older persons. To make this possible, the presence of risk factors and situations that potentially lead to falls must be evaluated and proper interventions must be defined. As described in the previous section (Section 2.3), there are several recognized risk factors for falling. A comprehensive assessment is then necessary so that a complete evaluation is done.

As suggested by clinical practice guidelines the multifactorial interventions should not be generically initiated for people aged 65 or older. Instead, these interventions should be reserved for aged persons presenting a risk of falling, which implies a previous screening phase for fall risk. Guidelines define how to recognize a high-risk status and also the most recommended risk factors targeted for intervention, based on evidence and strength of recommendation.

The following sections review the existing guidelines on fall prevention.

\subsubsection{Current Guidelines}

In a joint project of American Geriatrics Society (AGS), British Geriatrics Society (BGS) and American Academy of Orthopaedic Surgeons Panel on Falls Prevention, a guideline was developed and published in 2001 on the Journal of American Geriatrics Society. The principal aim of this document was to assist health care professionals in their assessment of fall risk, including fall evaluation/comprehensive assessment procedures and interventions to prevent falls [20].

The assessment and management of falls as described by this guideline is summarized in Figure 2.6.

According to this guideline, all patients should be asked about falls in the past year and everyone who reported a single fall should be observed for gait/balance problem. Everyone who presented for medical attention because of a fall or reported recurrent falls in the past year should be referred to a fall evaluation. Fall evaluation should include the assessment of history of fall circumstances, medications, medical problems, mobility levels and examination of vision, gait, balance, lower extremity joint function, basic 
neurological function and basic cardiovascular status. After assessment, a multifactorial intervention should be performed [20].

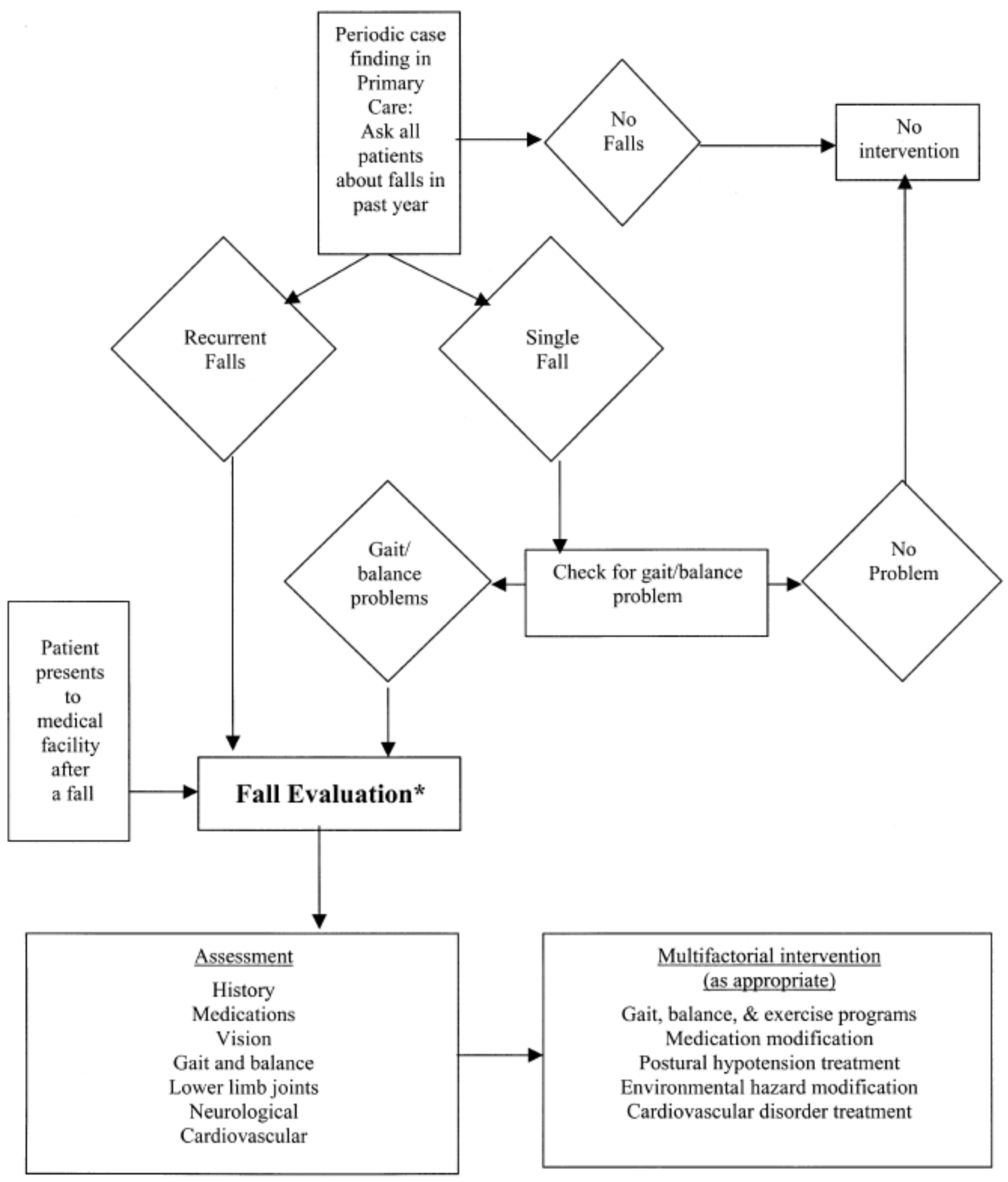

Figure 2.6: Assessment and Management of falls. Source: [20].

Later, in 2009, the same two organizations, AGS and BGS, published an online guide. A summary of guideline is presented on Figure 2.7. In contrast to the previous guideline, this makes a clear distinction between fall risk screening and assessment phases [27].

During the screening phase, simple questions about recurrent falls, frequency of falling and difficulties in gait or balance are used. Any positive answer to the screening questions puts the person into a high-risk group, and a multifactorial assessment of risk is recommended. If a single fall was reported in the past 12 months, an evaluation of gait and 
balance should be performed. This evaluation intends to identify the individuals who may benefit from a multifactorial assessment of risk and those who not [27].

The referred multifactorial fall risk assessment intends to identify the factors that put the person at risk of falling and also the most appropriate interventions to modify those risks, aiming to reduce falls. Multifactorial assessment should include all the factors identified in the later guidelines (i.e. cf. [20]), also including an examination of feet and footwear, muscle strength, environmental assessment, functional assessment of activities of daily living (ADL) skills, individual's perception of own ability and fear related to falling [27].

A guideline was also developed in London by The National Institute for Clinical Excellence (NICE), in 2004. Among the main areas examined by the guideline it can be found an analysis of the evidence for the factors that increase the risk of falling, the most effective methods of assessment and identification of older people at risk of falling and some interventions and preventive strategies [1].

NICE guideline cover the care of people in community. Hospitalised patients or people who are confined to bed for the long-term are not covered by guidelines.

Like the previous cited guidelines, NICE advices a multifactorial fall risk assessment if some gait or balance abnormalities are present, if people presented for medical attention because of a fall or if recurrent falls were notified. They recommend a population-based screening of falls and falls risk, which typically occurs when patients contact with health care professionals. All adults who are over 65 should be screened at least annually for fall risk and routinely asked to identify if they have fallen in the past. The multifactorial assessment should be done by experienced falls clinicians in a dedicated falls clinic. Interventions should be done in order to promote independence and improve physical and psychological function [1].

Guidelines also recommend that patients and their carers are informed about their risk of falling, mainly when they are transferred between care settings or when they are discharged from hospital settings. All health care professionals dealing with patients at high risk should develop and maintain competences in falls assessment and prevention [1].

In 2005, in France, another good practice guide on falls prevention was published [19]. This guide is directed to elderly living at home and also identifies two distinct phases on the management process: screening for risk of falls and comprehensive assessment. The guide further details the screening phase, by classifying as high risk, moderate risk and low (or no) risk of falling (see Figure 2.8).

According to this guide the screening includes the evaluation of history of falling and gait or balance impairments. If the person had already fallen and presents gait or balance impairments, he/she has a high risk of falling and a comprehensive assessment is recommended. A moderate risk is identified if history of falls or gait/balance impairments 


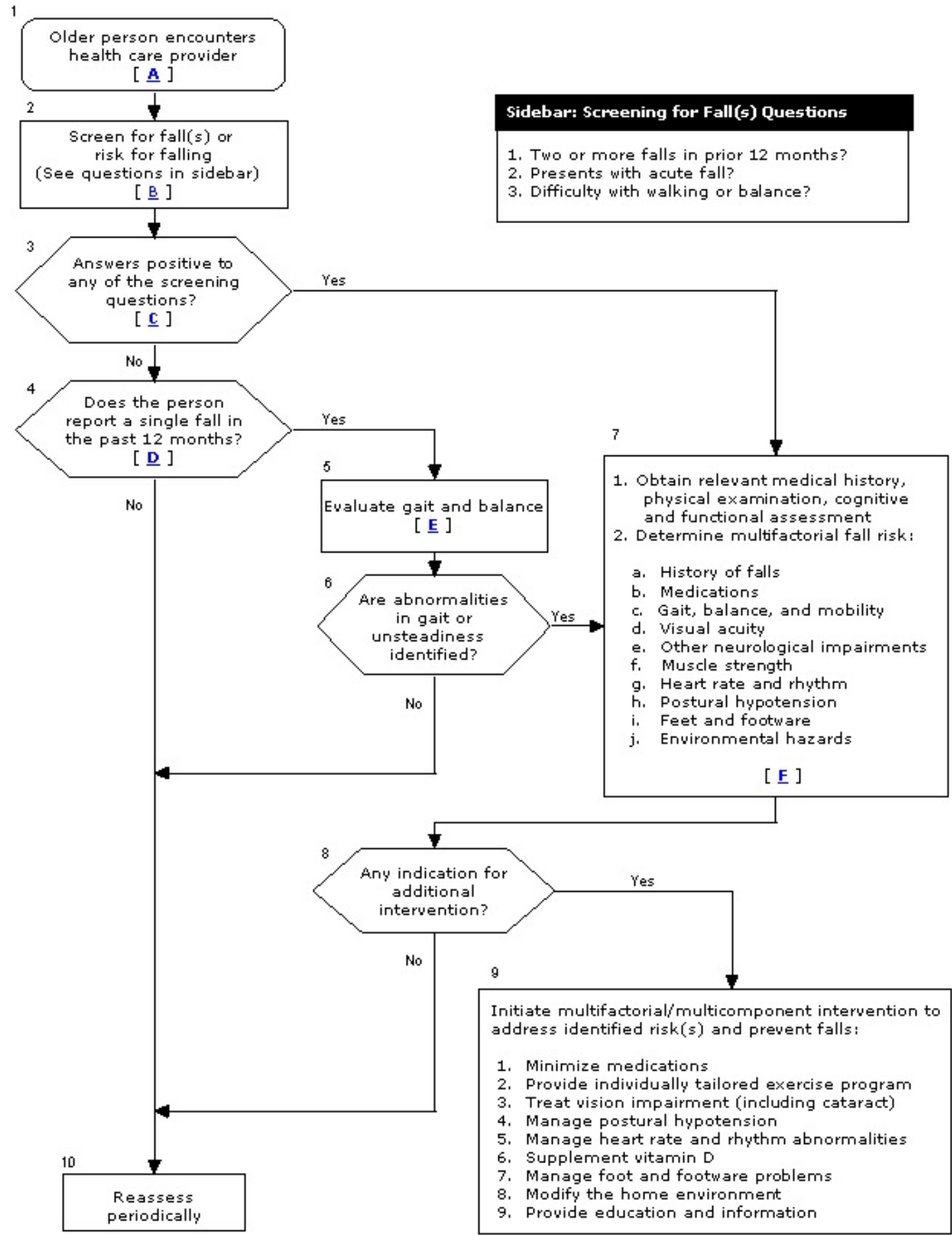

Figure 2.7: AGS/BGS Clinical Practice Guideline. Source: [27].

are identified. In this case, comprehensive assessment is not obligatory, but a minimal assessment of some specific risk factors should be performed, which include balance, gait, physical activity, diseases, medication and dangers at home. Low risk of falling is present if there's no history of falls and no gait or balance impairments. In this case, a minimal 
assessment is also recommended and any detected risk factors should be addressed with specific interventions [19].

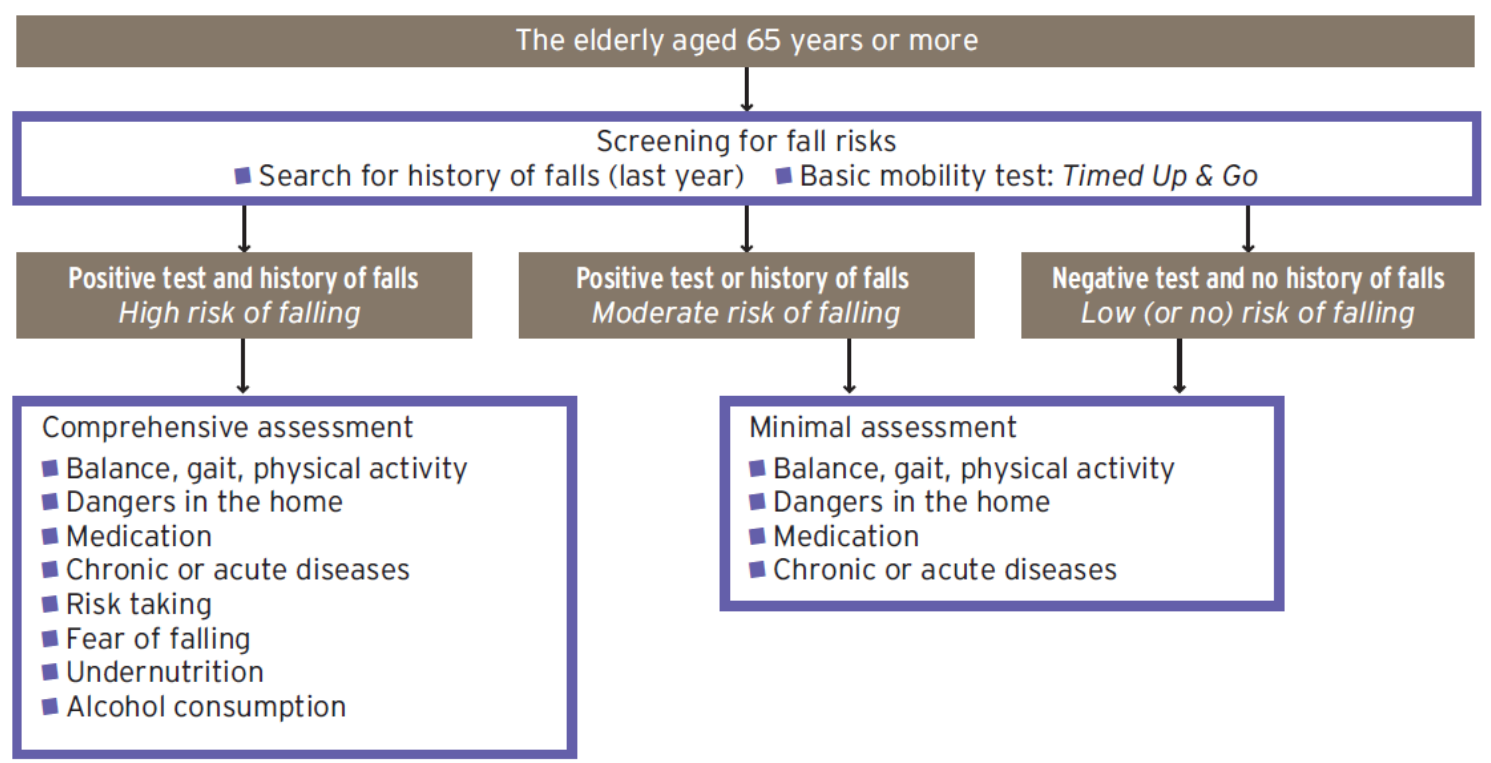

Figure 2.8: Screening and Assessment of fall risk. Source: [19].

In 2007, an article published on the Journal of American Medical Association reinforced the idea suggested by previous guidelines that multifactorial interventions should be reserved for high-risk patients. According with the authors, this status is identified by a history of recurrent falls, a fall requiring medical attention or an abnormality of gait, balance or both. This screening phase has only the purpose of deciding who should receive a multifactorial intervention. They recognize that a refinement of this approach would be to quantify a patient's global risk of falling and to intervene if the risk exceeds a certain threshold [25] .

\subsection{Clinical tools/technologies}

Several tools exist for screening and assessment of fall risks. Although several different classifications for tools are proposed by different authors (cf. [28], [29] and [30]), to maintain the consistency with guidelines presented on the last section (Section 2.4), tools will be primarily divided into fall risk screening tools and fall risk assessment tools, which comprises a comprehensive fall risk evaluation.

A complete description about fall screening and assessment tools is provided on the next sections. Each tool is described in detail in Appendix A. 


\subsubsection{Fall risk screening tools}

Fall risk screening has the purpose to identify aged people with a high-risk status who should receive a multifactorial intervention. As stated by recommendations this can be done through the identification of history of falling in the last year or abnormalities of gait, balance or both [25]. In this phase, a quick and simple gait and balance assessment is privileged, to facilitate the integration of screening as a common practice [19].

It is recognized that measures of motor function capacities are powerful predictors for risks of falling [19]. Assessment of gait and balance is therefore the mainstay of screening [25].

There are different options for measuring and attributing risk. Some tests reveal gait and balance aspects, others only a single aspect.

Persad et al. (2010) classify the falls screening tools into three different groups [28]. The first group, the self-reported scales, rely on the screening for fall history. The screening of one or two aspects of gait and balance is done using another group of tools, which is the single-task performance measure. These tools are usually simple and fast, and the performance measure (e.g. time to complete the task or the number of steps required) is easy to assess. Other qualitative measures are also evaluated by doctor, based on the observation of the task [7, 31, 32]. A popular test of this kind is the Timed Up and Go Test (TUGT), which is highly recommended by [19]. Other examples are the One-Legged Stand (OLS) Test, Functional Reach Test (FRT), Sit-to-Stand transfer with five repetitions (STS5), the stepping tests (e.g. Rapid Step Test (RST), Four Square Step Test (FSST), Maximal step length (MSL)) and the Comfortable Gait Speed (CGS). A detailed description of these tools is presented in Appendix A.

A more complete evaluation of fall risk is done incorporating measures of balance, gait and fall history, which is provided by multiple-task measures [28], e.g. Performance Oriented Mobility Assessment (POMA), Berg Balance Scale (BBS), Dynamic Gait Index (DGI) and Physiological Performance Assessment (PPA).

Considering the importance of balance-based tools for fall risk screening, Mancini and Horak (2010) divided these tools into three different classes: functional assessments (used to determine if a balance problem exists), systems/physiological assessments (to determine the underlying causes of balance deficit), and the quantitative assessments (that use computerised technology) [29]. The latest will be further detailed on the next paragraphs.

\section{Technologies}

Instrumented assessments have been developed in order to detect more subtle problems with gait or balance and provide objective measurements of individuals fall risk [33], being an improvement of the methods based on observation, which are subjective [11]. 
The most simple commercial devices calculate some parameters, but their interpretation has to be performed by the clinician or the researcher [11].

Force platforms and cameras are usually used for quantitative analysis of gait, balance and postural control $[34,35,36]$. These equipments are expensive and require technically skilled personnel to deal with them. They are not applicable if there's some limitations on space, time and funding, which frequently happens in clinical practice, limiting their use as routine [11, 36, 37, 38, 39].

Force platforms measure the ground reaction forces generated by a body that is standing on or moving across them, for the estimation of the centre of pressure (COP) [34]. They are highly accurate, but typically too costly to be used in standard falls clinics. The administration of the motion analysis requires an expert, that is also required to interpret the results [11].

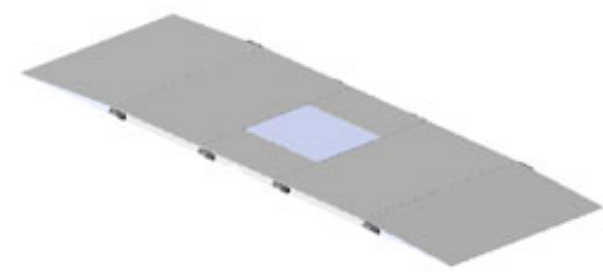

Figure 2.9: Walkway with one force-plate. Source: [40].

An example of a force-plate is that provided by AMTI Force Platforms [40]. AMTI Force plates measure forces and moments applied to its top surface. They can be used individually or arranged in a walkway format to collect multiple steps (Figure 2.9). They offer solutions for gait, balance and sports applications, and also portable and mounted models. The portable platforms are lightweight, rugged and economic, being designed to be used when there's no available lab space for a permanently mounted installation [40].

The optical motion capture systems, referred for gait analysis, capture some markers applied at specific anatomical points of the body, to compute underlying motion of body segments [11].

One example of such as system is the CODAMotion (Figure 2.10) [41]. It is a portable system used for real-time 3D movement measurement [41].

There are also pressure sensitive floor mats or walkways, which allow the measurement of pressure distribution under the feet. They can be defined as gait walkways or gait mats. The gait walkways are long, usually with 3 to 5 meters, and measures the static and dynamic pressure and force over several steps using a low profile floor walkway, for example the GaitRite Walkway (see [42]). Gait mats are short (i.e. less than 1 meter) and captures dynamic pressure as person walks over it and deviations in COP while a person performs a series of tasks. An example of such a system is the Tekscan MatScan (see [43]) [11]. 


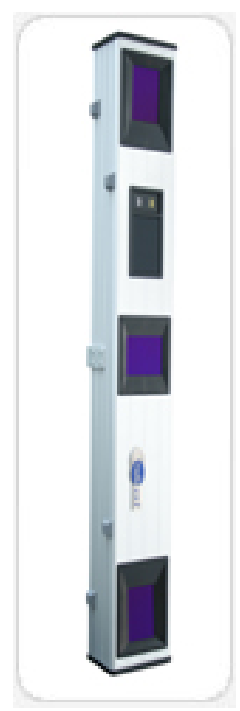

Figure 2.10: Codamotion 3D measurement instrument. Source: [41].

Gait walkways and gait mats require minimal training to use and are quick to set up and record the data. They are commonly found in falls clinics. Although they can be moved, they are not particularly portable [11].

Body Sensor Networks (BSN) are recently being used to assess gait and balance problems. They have the advantage of being portable, and therefore they can be used in a doctor's office or at home. BSN can be formed by one or a combination of wearable sensors, such as accelerometers, gyroscopes, magnetometers and insoles. It can also include systems for logging, transmission or processing of recorded data [11].

The insoles are pressure sensors that are incorporated into shoes, to measure force distribution on a patient's foot to assess balance problems [11].

Some commercial devices using sensors already exist, such as the DynaPort (see [44]). They offer some solutions for clinical assessment of physical performance like locomotion, postural control and sit-to-stand transitions [44].

The DynaPort GaitTest uses a triaxial accelerometer that is attached to the waist and enables to perform gait analysis and identify impaired gait. Through this system, the major gait cycle events, such as heel strike, toe off and double support are detected using the algorithm described by [45]. Based on these events, advanced parameters, such as step time asymmetry, bilateral coordination and stride time fluctuations are calculated [44].

The DynaPort SitToStandTest enables a qualitative evaluation of sit to stand action. It uses the triaxial accelerometer and triaxial gyroscope to measure accelerations and angular velocity, enabling the identification of events during standing up and sitting down and the determination of the trunk angle [44].

The DynaPort SwayTest is also based on body fixed motion sensor and enables the evaluation of a person's balance through the direct following of the movement of the 
centre of the mass of the individual. The traditional systems use force platforms that estimate the displacement of the centre of pressure [44].

\subsubsection{Fall risk assessment tools}

Multifactorial interventions are reserved for high-risk patients. It consists of an in-depth assessment to discover the potential causes of falls in a particular person, so that enough detail exists to make right decisions about the factors that need intervention [25].

The multifactorial assessment of fall risk can include all the risk factors for falling identified as potential causes to falls. Guidelines identified the most recommended risk factors to include in the comprehensive assessment phase for fall risk prediction (cf. Section 2.4). A detailed description of different ways to assess risk factors is provided on the next paragraphs.

\section{Falls history}

Search for history of falling can be done questioning the person about falls within a past frame, i.e. 6 or 12 months. An exploration of the context of falls should also be done, regarding frequency, surrounding circumstances, activities and falling consequences [19, 28].

Some formalized questionnaires are now included in some tools to access the history of falling. The Falls Risk for Older People in the Community scale (FROP-Com) is one example of a structured questionnaire that doesn't require any special equipment. The tool consists of 13 rated risk factors, including the history of falls [28]. A complete description of this tool can be found in Appendix A.

A problem with history of falling assessment is that sometimes the person may not remember minor falls in the past, which is especially true if the patient is experiencing memory problems affecting the capacity to remember past events. Also, the individual's perception of what constitutes a fall can be quite variable [28].

\section{Chronic and acute diseases}

There are some diseases that are known to increase the risk of falling. Beyond the age of 65 , these diseases are frequent and an evaluation is recommended in order to diagnose chronic or acute diseases [19].

Special attention should be give to the presence of arthrosis, foot deformities, visual disorders, proprioceptive disorders, neurological disorders, cognitive impairment, hypotension, dehydration, undernutrition, depression and infections, which are frequently associated with falls [19].

The evaluation of diseases must be performed by a physician (i.e. a generalist, internist or a geriatrician), possibly with the assistance of other healthcare professionals, such as 
specialists (e.g. neurologists, psychiatrists, cardiologists), nurses, physical therapist or occupational therapist [19].

\section{Gait and Balance Examination}

Several tools exist to evaluate gait and balance.

Ordinal scales requiring an observer that subjectively rates the person's ability to perform some tasks are commonly used to assess balance. This kind of scales include the BBS, POMA and the Balance Evaluation Systems Test (BESTest).

Other functional performance tests, such as the TUGT, FRT or the Turn $180^{\circ}$ are also used in falls clinics.

Highly accurate instrumented assessments are becoming increasingly common in falls clinics and frequently they are preferred for a quantitative evaluation of gait and balance. These instruments enable a more detailed evaluation of problems. As described previously, these instruments can include pressure sensitive floor mats, force-plates, optical motion systems and BSN, requiring an expert to administer the tests and evaluate the results [11].

\section{Medication}

Prescribed drugs and self-medication should be reviewed. Attention should be paid to the use of four or more drugs, the use of drugs known to increase the risk of falling and possible drug interactions [19].

\section{Basic and Instrumental Activities of Daily Living}

Basic ADL typically include toileting, feeding, dressing, grooming, ambulation and bathing. Instrumental activities of daily living (IADL) include the use of the telephone, shopping, preparing food, housekeeping, doing laundry, transportation, managing medications and handling one's own medications [25].

An impairment in these daily activities can put the person in an increased risk of falling. Consequently, some questionnaires exist to simply ask whether the patient needs or does not need help with any of these activities [25]. The Barthel index (BI), Katz ADL and Lawton IADL are some examples of these kind of scales and are described in detail on Appendix A.

It is recommended to assess the risks taken in the person's daily activities, which include an assessment of environmental risks [19]. 


\section{Fear of falling}

In contrast with previous approaches that simply consisted of asking whether someone was afraid of falling, new measures of balance confidence were developed to provide a sensitive measure of fear of falling, e.g. Activity-Specific Balance Confidence (ABC) Scale, Falls Efficacy Scale (FES) and Modified Falls Efficacy Scale (MFES) (details provided in Appendix A).

Balance confidence is defined as the belief that the individual has the capability to perform an activity or action. The measures of self-confidence are strongly linked with independence in daily and social activities [46].

\section{Behaviour}

There are some behaviours that are considered to constitute risk taking.

The doctor must encourage the person to maintain a safe behaviour in his/her daily activities, preventing from high-risk situations. These include hurrying, climbing onto a chair, walking with hands on pockets, using a cane or walker improperly, wearing inappropriate footwear, neglecting the use of eyewear, going up and down the stairs in inappropriate footwear, not using the handrail and leaving objects on the steps.

Alcohol consumption must also be evaluated, regarding the amount habitually consumed, the frequency and the context of consumption [19].

Behaviour must be evaluated having in consideration the person's real capacities.

\section{Dangers in the home}

There are numerous tables for identifying fall risks in the home, by identification of obstacles, e.g. the Home Falls and Accidents Screening Tool (HOME FAST). Some of these tools are self-evaluations, e.g. the self report version of the HOME FAST described in Appendix A.

Research suggests that it may be preferable to assess individuals during their daily activities in home. Based on these activities, the actions intended to modify the home are more pertinent and result in improved fall prevention [19].

Assessment of functional capacity in the living environment is also highly recommended. This will allow for the verification of the adequacy of the environment to person's real capacities [19].

\subsection{Falls prevention}

The identification of individuals at a high risk of falling is important for the design of fall prevention programmes [17]. 
The risk factors for falling are diverse and many of them are modifiable. Then, the preventive intervention programmes are usually based on multiple interventions and need to target the factors assigned as risk factors for falling. They can include exercise programmes for muscle strengthening and balance retraining, Tai Chi group exercise intervention, education programmes, medication review and environmental modification in homes or institutions $[1,20]$.

However, there are other key changes in addition to prevention that can be performed to reduce the number of falls and their impact. These include the improvement of diagnosis, care and treatment of those who have fallen, rehabilitation and long-term support and ensuring that patients and their carers are receiving proper advice on prevention of falling [1].

The reduction of falls in a variety of settings when applying a multi-factorial assessment and a prevention programme is estimated to be by around $25 \%$ [4].

\subsection{Recent studies on fall risk prediction}

Previous studies focused on risks factors identification and development and validation of scales and functional performance tests. This was performed in several different settings, where incidences and risk factors differed.

More recently, authors are trying to improve these methods by quantification of measurements, so that new technologies (e.g. force plates and optical motion systems) were developed for gait and balance assessment. Although these systems were able to detect more subtle problems with balance or gait, some restrictions were identified on their use: they are expensive, not portable, and frequently poses limitations on space, time and funding, limiting their use as routine in clinics [11, 36, 37, 38, 39].

Nowadays, the studies are focusing on detection of gait or balance problems using wearable sensors, which are demonstrating to have lots of advantages in relation to the previous methods, being a promise on the improvement of fall prevention strategies [11].

Recent studies focus on the measurement of parameters from sensors signals, based on the existing medical validated tests for fall risk screening (e.g. cf. [32], [47] and [7]), directed routines with a series of movements and assessment tasks (e.g. cf. [10], [48], [6] and [49]) and walking patterns and gait analysis (e.g. cf. [50] and [31]). They evaluate gait, standing balance, sit-to-stand transfers and other ADL, which can be used to evaluate the risk of falling of an individual [6].

The study performed by Marschollek et al. (2008) used a dataset that was obtained by sensory measurements during the performance of TUGT [32]. An implementation of this test could be done as suggested by Ni Scanaill et al. (2010) [11], Figure 2.11. 

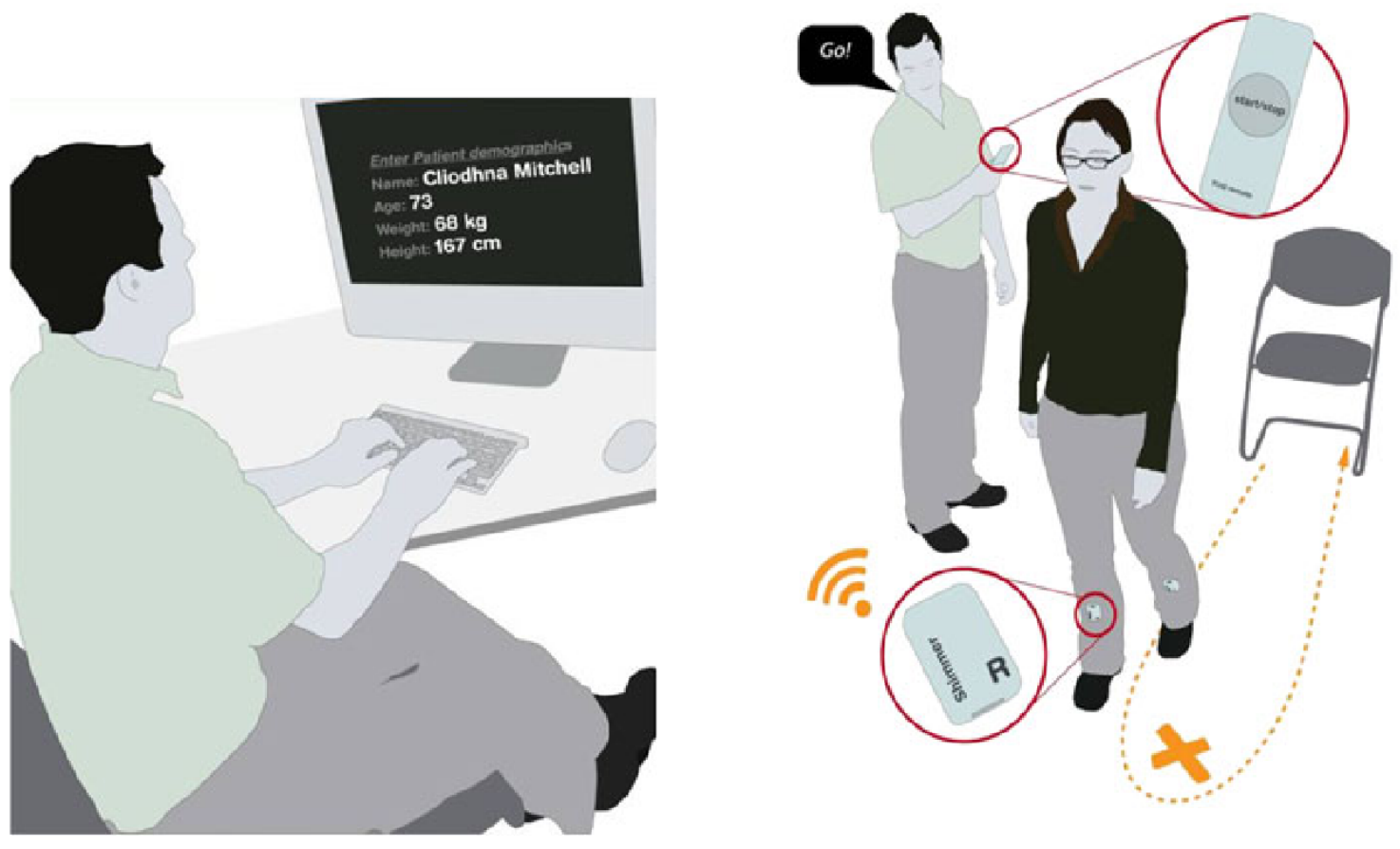

Figure 2.11: Illustration of implementation of the TUGT using inertial sensors. The doctor enters the patient's demographic details and then administers the test. Source: [11].

Greene et al. (2010) also used sensors to evaluate TUGT [47]. Two kinematic sensors containing a triaxial accelerometer and a triaxial gyroscope were attached to the anterior part of each shank (as illustrated on Figure 2.11). Figure 2.12 illustrates the signal obtained from left and right shank angular velocity.

King et al. (2010) has used only one e-AR (ear-worn activity recognition) sensor with an embedded 3-axis accelerometer to extract data during the performance of TUGT and POMA [7]. Some parameters of POMA could not be addressed from this sensor alone, since, for example, it was impossible to determine if the patient used his/her arms to rise from the chair. For the TUGT, in turn, as it is mainly based on the time taken to complete the test, the sensor performed well and demonstrated great potential to extract further data from the gait to provide a more depth analysis of the level of risk of the individual [7].

In the studies performed by [10], [48] and [6] they used a directed routine that included a set of short movements to be self-administered and performed periodically. The following assessment tasks were included: Near-tandem standing balance (NTSB), TUGT, Alternative Step Test (AST), STS5 and Simple Reaction Time. An example of the data obtained by Narayanan et al. (2007) [10] when using a waist mounted triaxial accelerometer during the performance of five different tasks is presented in Figure 2.13.

Caby et al. (2011) used an accelerometer network comprising 10 sensors, that were attached to the knee, ankle, elbow, wrist and shoulder blade of each side. Several fea- 

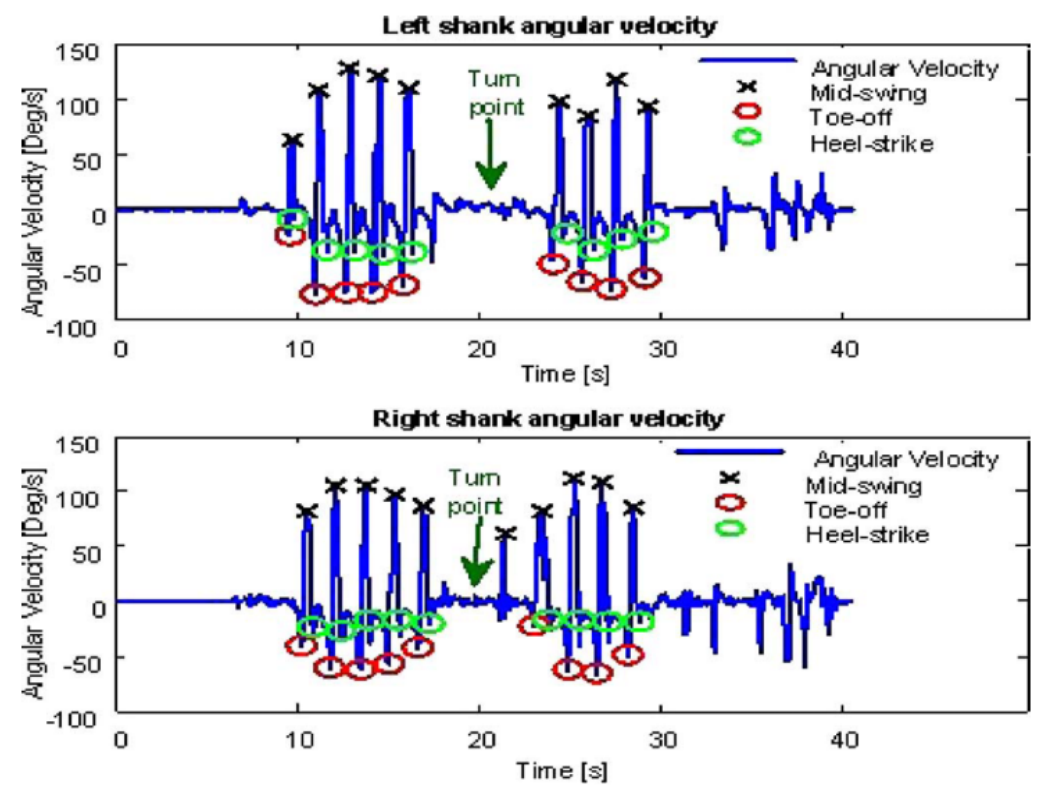

Figure 2.12: Example of a left and right shank angular velocity signals while performing TUGT. Source: [47].

tures were extracted from acceleration signals when performing different tasks, including a $25 m$ walking test, the TUGT, PPA and the OLS. At the end, the features were used by classification algorithms, in order to discriminate people from fallers and non-fallers groups [49].

Other studies were based on the analysis of gait. Bautmans et al. (2011), for example, has used the DynaPort MiniMod 3D accelerometer [31]. It was positioned on the pelvis of subjects while they were instructed to walk comfortably along $18 \mathrm{~m}$.

Allet et al. (2008) performed an evaluation of gait using different pathways: 50m of a tarred pathway, $50 \mathrm{~m}$ of grass and $20 \mathrm{~m}$ of cobbled stone pathway [39]. Four miniature gyroscopes were used attached to each shank and thigh during walking at a preferred walking speed.

Other movements were also identified as predictors of risk of falling [6].

Rising from a chair, for example, has been recognised as a mechanically demanding functional task usually performed during daily activities. The sit-to-stand ( $\mathrm{SiSt}$ ) and stand-to-sit (StSi) transitions have also well-known characteristics [51].

During the rising phase four tasks occur: forward lean of the trunk, upward movement of the trunk, the extension of the knee and backward movement of the trunk (stabilisation). In the descending phase there is a forward and downward movement of the trunk, flexion of the knee and finally a backward movement of the trunk for stabilisation. The strategies of SiSt and StSi transitions differs as the person ages, e.g. older persons need greater forward lean of trunk and usually perform the activity of rising more slowly [51], c.f. 

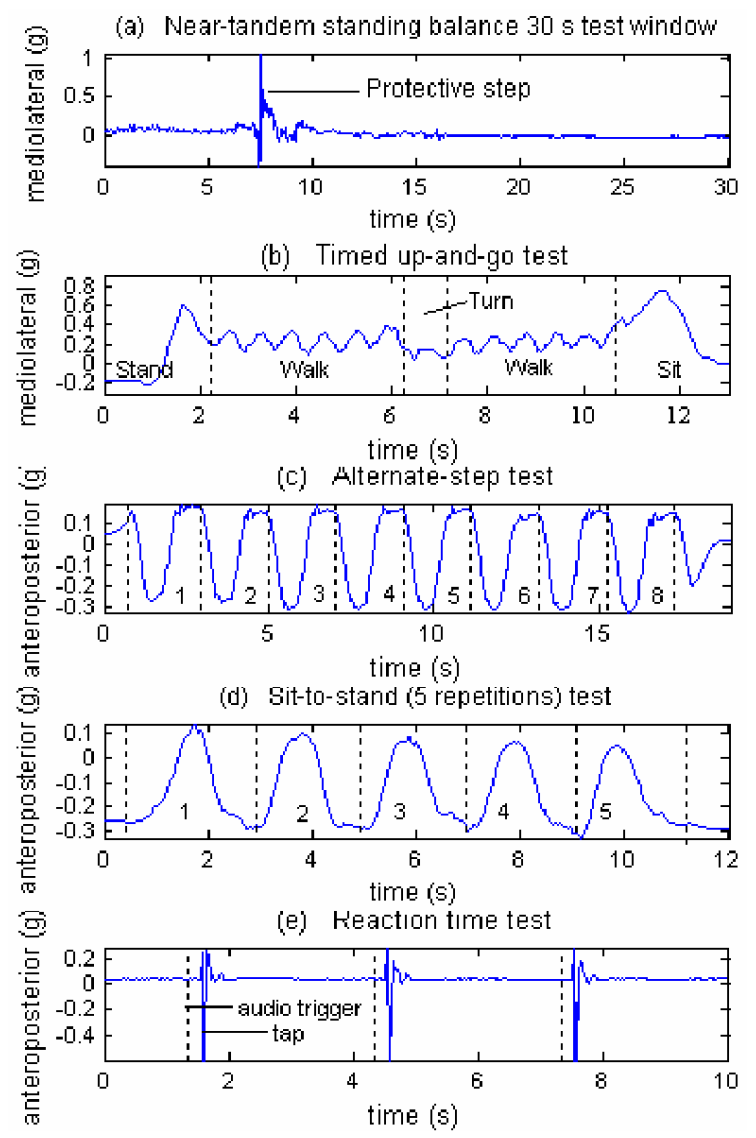

Figure 2.13: Example of the data obtained when using a triaxial accelerometer based wearable unit during the performance of tasks of a directed routine. Source: [10].

Figure 2.14.

Some parameters can be extracted using gyroscope during the SiSt and StSi activity, which include the trunk tilt (angle between the vertical axis and the anterior wall of the subject's thorax, Figure 2.15a) and the time and duration of postural transition, as described by [52]. In this study the gyroscope was attached with a belt in front of the sternum, resulting in a variation of $\sin (\theta)$ presented on Figure 2.15b.

Acceleration signals can equally be extracted during SiSt and StSi postural transitions (see Figures 2.16 and 2.17) [53].

Najafi et al. (2002) recognise that gyroscope can be attached anywhere to a body segment as long as its axis is parallel to the mediolateral axis, because the angular rotation will be the same in all segment [52]. Authors also state that angular velocity is less noisy than the acceleration signal extracted from accelerometer, since this signal involves higher frequency components. The gyroscope signal doesn't have the influence of gravity acceleration, which in turn happens on accelerometer data [52].

Giansanti (2006) has used a wearable device with three mono-axial accelerometers 


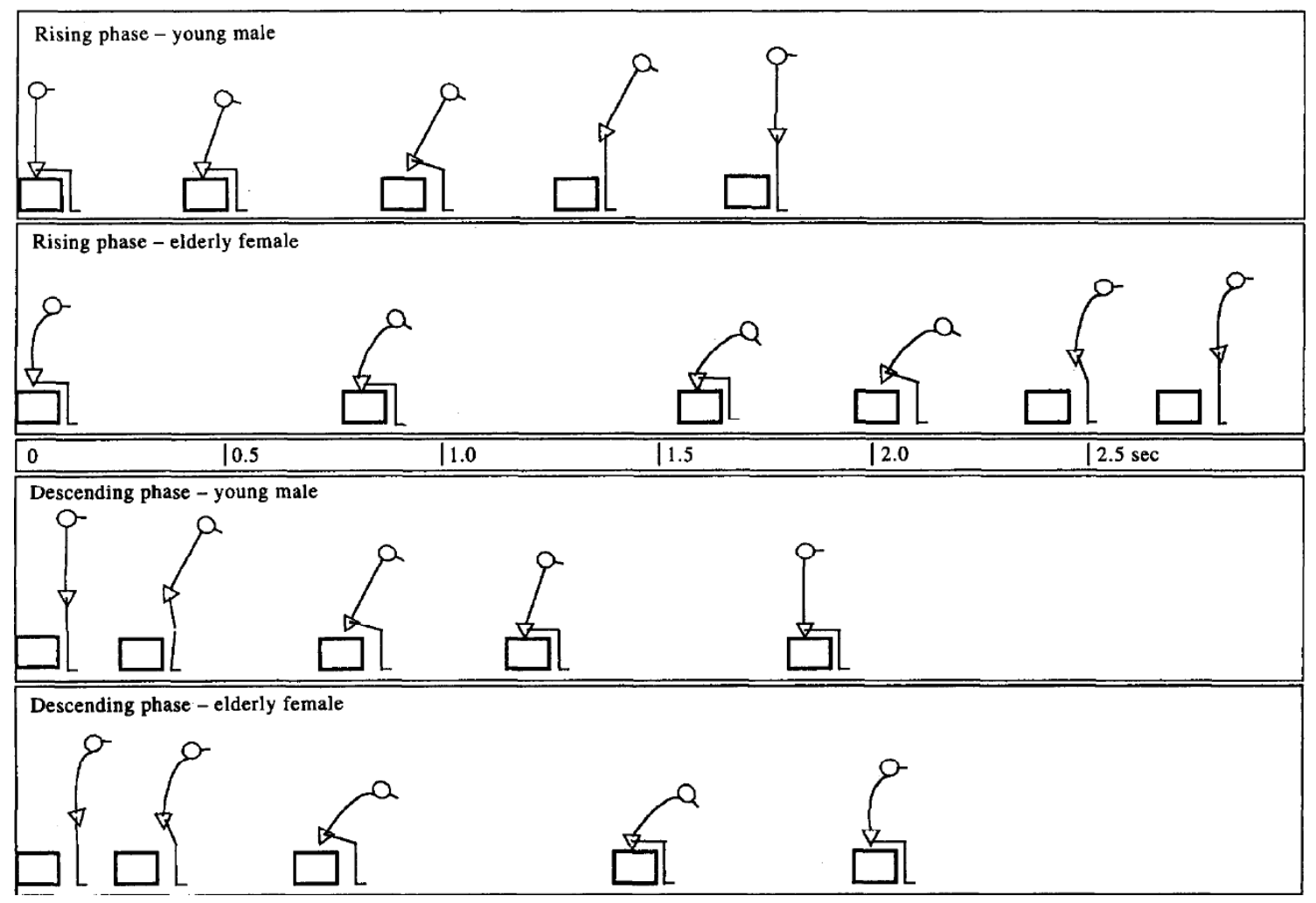

Figure 2.14: SiSt and StSi transitions in a young male $v s$ an elderly female. Source: [51].

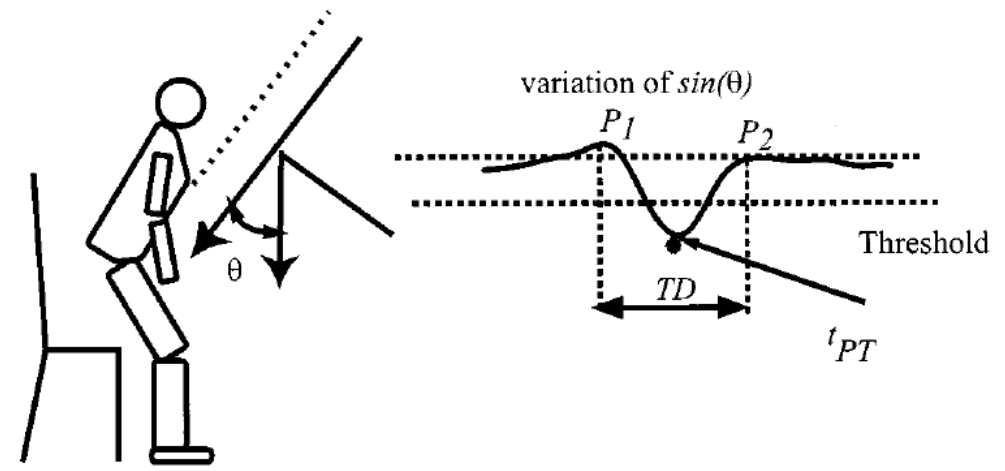

(a)

(b)

Figure 2.15: SiSt transition. (a) Trunk tilt (represented by the angle $\theta$ ); (b) Variation of $\sin (\theta)$. $P 1$ represents the beginning of tilt down and $P D$ represents postural transition duration time from $P 1$ until the end of the tilt back (P2). Source: [52].

and three gyroscopes to record the standing sway of subjects under different conditions: eyes open on a solid surface, eyes open on a foam cushion surface and eyes closed on a foam cushion surface. From these data, they extracted several parameters, and, through classification techniques, they discriminated the older people with high fall risk [54]. 


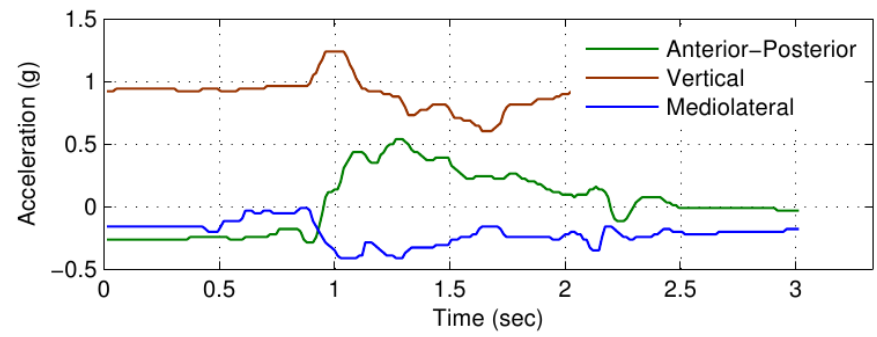

Figure 2.16: Acceleration signals during SiSt transition. Source: [53].

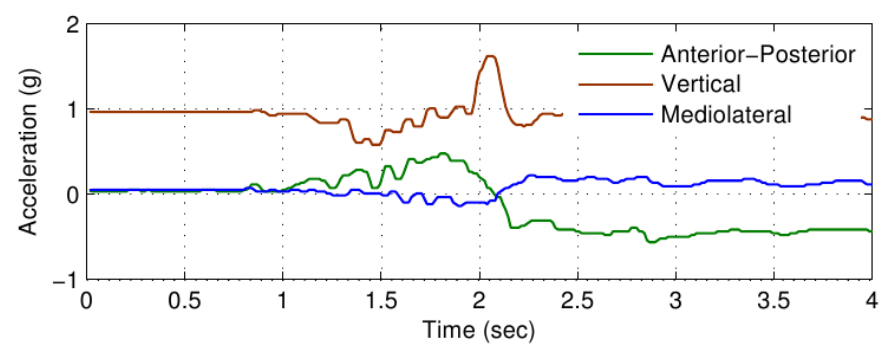

Figure 2.17: Acceleration signals during StSi transition. Source: [53].

As already perceived by the referred studies, the use of parameters while performing different activities are used to detect the risk of falling, discriminating those at no risk and at risk. This can be done through the quantification of the same parameters used in assessment scales, like the time to complete the test in TUGT (see [7]), quantification of mobility or gait parameters associated with fall risk (cf. [55], [56], [57], [58] and [59]) or using machine learning techniques, with the ground truth provided by clinically accepted scales (cf. [47], [32], [48], [6] and as suggested in [50]).

Results from Laessoe et al. (2007) support the idea that fall risk cannot be predicted solely assessing a physical performance, since falling is a complex phenomenon with a multifactorial nature [60]. Some articles suggest the quantification of the patient's global risk of falling, so that an intervention is done when the risk exceeds a certain value (refer to [61], [25] and [62]).

Global risk's quantification can be achieved by accessing different risk factors (i.e. building a risk profile through a multidimensional risk screening) and then combining them through the attribution of different weights to each risk factor. As a result, a likelihood of falling should be calculated $[61,62,25]$. The calculus of a likelihood of a fall implies the use of logistic regression methods to estimate the regression coefficients of each predictor of falling, as suggested by different articles (c.f. [61, 62]). Ganz et al. (2007) used likelihood ratios to calculate the probability of fall for a particular patient [25].

Indeed, self assessments can allow people to become more aware of the actions that 
are needed to improve their health, encouraging them to be active in their own health care [26].

The risk profile can be useful to design some useful interventions targeting the detected risks which are modifiable. It can also be useful to collect information regarding individual's risk factors history, that can contribute to the study of falls. The routine multidimensional fall risk screening could then be a major strategy in fall prevention [63].

\subsection{Gait phases}

The human bipedal walking, referred as gait, is a repetitive and complex activity. Four main sub tasks of gait can be identified for a successful walking. Initiation and termination of locomotor movements, generation of continuous movement to progress in a direction, maintenance of equilibrium and adaptability to environmental changes are characteristics of gait [50].

Gait cycle can be divided into different phases. An illustration of a complete gait cycle is provided in Figure 2.18.

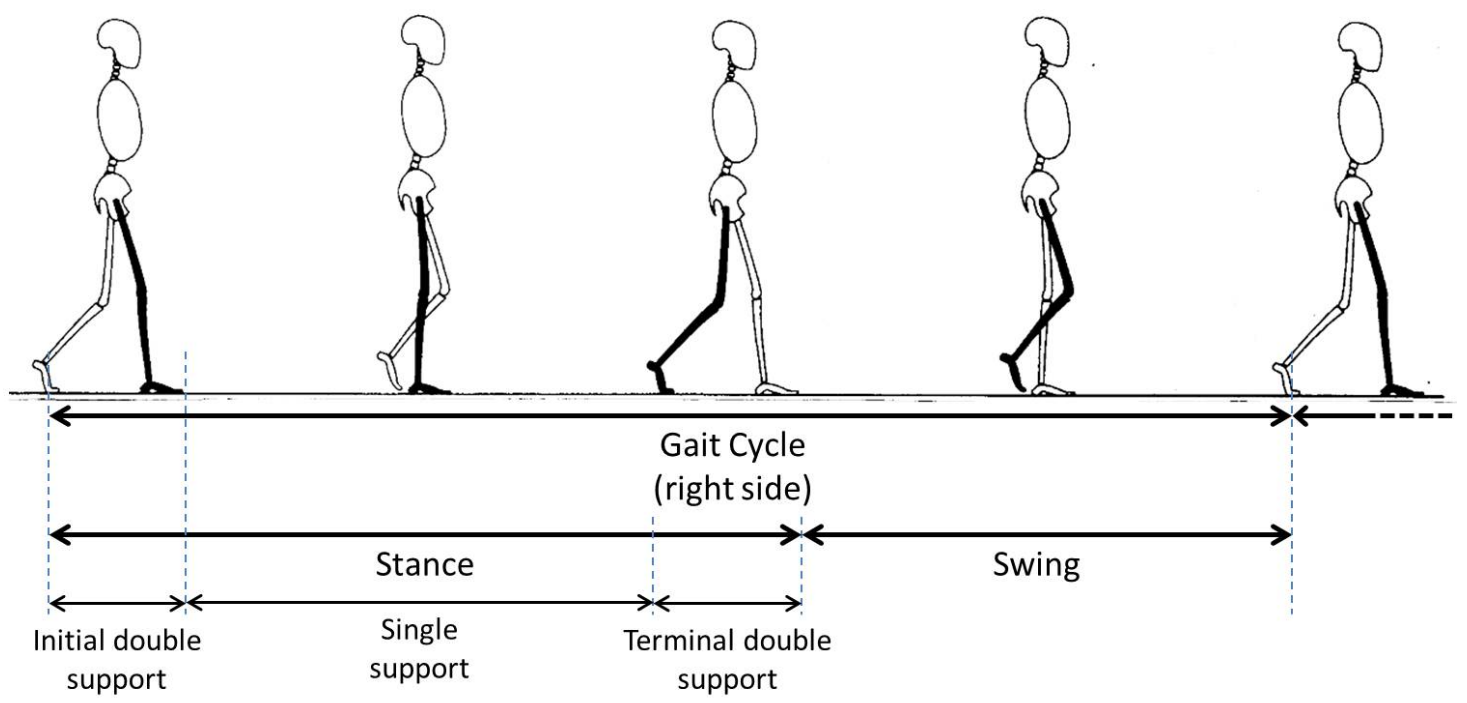

Figure 2.18: Gait cycle. Adapted from [64] and [65].

A single gait cycle is called stride and is defined as the time between the heel's first contact with the ground and the next contact of that same heel $[47,50]$.

The stride comprises two phases: stance and swing. The stance phase is the interval in which the foot contacts the ground, i.e. the time between a heel strike and a toe off on the same foot. The swing phase begins when the foot leaves the ground and is defined as the interval in which the foot is not in contact with the ground, i.e. the time between toe off and heel strike of the same foot $[47,66]$. 
The time when the both feet are in contact with the ground is the double-support time [66]. The single support time of the right foot equals the swing duration of the left foot, and vice versa [47].

Gait has many measurable characteristics, including velocity, temporal parameters such as step time, stride time and double support time and spatial parameters, such as step length and stride length [67].

\subsection{Using inertial sensors to evaluate gait}

The inertial sensors, such as accelerometers and gyroscopes, have recently emerged as an alternative to traditional methods, such as force plates and cameras, and have been used in recent studies to evaluate gait $[6,33,36,38]$.

These sensors are relatively inexpensive, lightweight, easy-to-use, non-obtrusive and portable, making them suitable for use outside the conventional gait laboratories. They can for instance be easily integrated into the clinical routine or in the patient's own environment, making measures that are representative of real life context [37, 38, 39].

Gait characteristics have frequently been recognised as efficient to identify people with increased risk of future falls [56]. Nowadays, there is a lack of uniform clinical gait protocols, so that the diagnosis of gait abnormalities are mainly based on examiner's expertise [56].

As referred, some commercial devices already exist for evaluation of gait using sensors, e.g. the DynaPort GaitTest (see Section 2.5).

In this section, the studies related to the evaluation of gait using inertial sensors are reviewed.

\subsubsection{Sensors signal analysis}

The lower limb movements are responsible for the displacement of pelvis, which supports and causes the displacement of upper segments, including the trunk [68].

With each step, the body weight is transferred from one leg to another, which causes an up and down movement of the pelvis during gait and coincides with its left-right movements. The pelvis also moves forward and downward with each step, displaying speed fluctuations in the direction of progression [68].

During the single support, it is expected that the forward acceleration increases, which is concomitant with an increase in body inclination (acceleration stage). During the transition from single to double-support (i.e. when a contra-lateral foot contact occurs) the body inclination decreases and the forward acceleration also decreases (decelerating stage) $[50,69,70]$. Thus, the instant of foot contact can be estimated from the peak forward 
acceleration in lower trunk that precedes a change in sign from positive to negative [45], as represented in Figure 2.19.

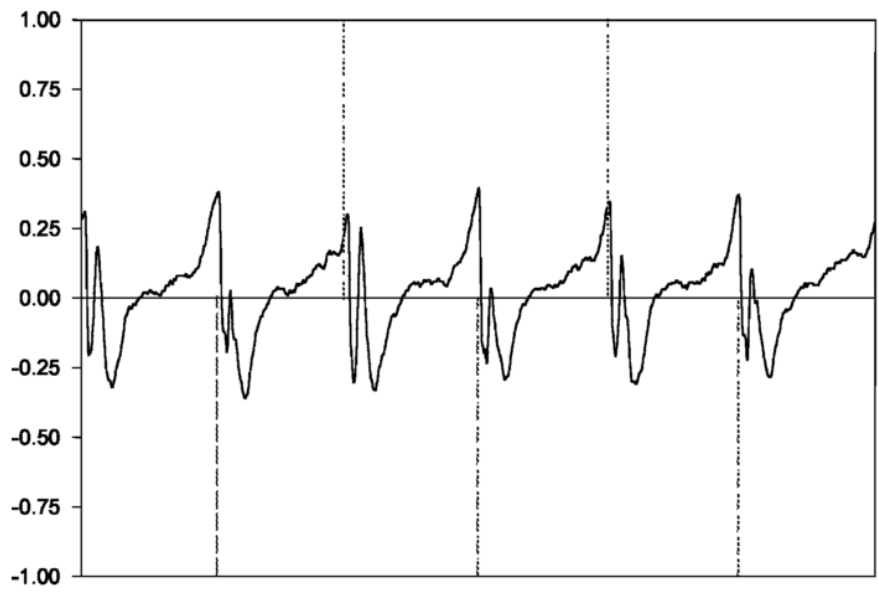

Figure 2.19: Antero-posterior acceleration signal at the level of pelvis. Dashed line represents instants of foot contacts. The scale is in units of gravity $(g)$. Source: [71].

In the lateral direction, it is also expected that an acceleration occurs to the right or left position, which occurs during left support phase and right support phase, respectively [69]. After a heel contact, there is an acceleration in the contralateral direction [71]. The rest of the signal is frequently described as highly irregular [71, 72]. Consequently, the discrimination between left and right foot contacts can be based on this lateral direction data [45]. On Figure 2.20 accelerations of pelvis in the lateral direction are presented.

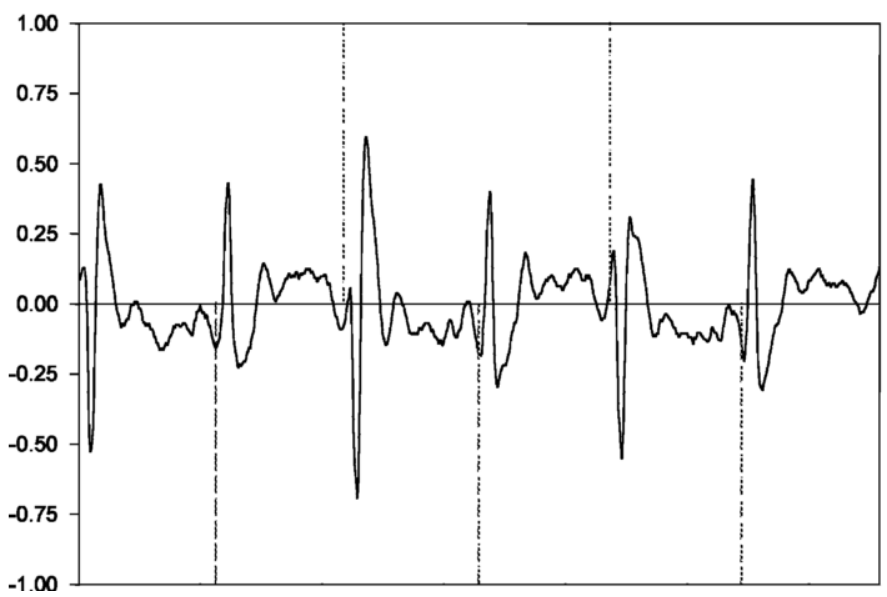

Figure 2.20: Medio-lateral acceleration signal at the level of pelvis. Dashed line represents instants of foot contacts. The scale is in units of gravity $(g)$. Source: [71].

In the sagittal plane, the COM follows a circular pattern during each single support phase [69]. During the acceleration stage, the increase of body inclination will cause that 
less gravity acceleration is projected onto the z-axis of the accelerometer attached to the body, and therefore a small vertical acceleration will be measured. In the deceleration stage, in turn, the decrease in body inclination leads to higher vertical accelerations [50]. As can be observed in Figure 2.21, the first valley will correspond to a heel contact and the second valley to final contact of the same foot $[72,73]$. After heel contact, a rapid upward acceleration is observed [71].

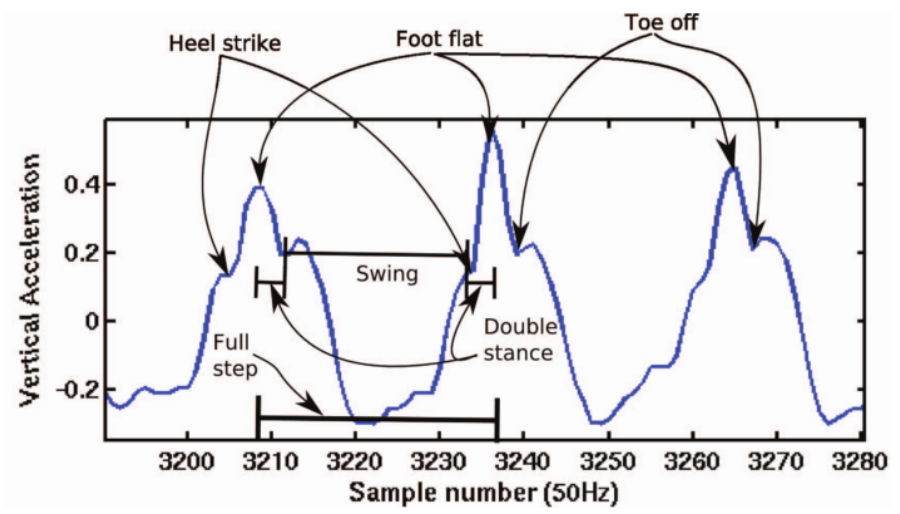

Figure 2.21: Vertical acceleration signal at the level of pelvis. Source: [73].

Acceleration signals are also described at the level of the head [71, 74]. In short, the vertical, antero-posterior and medio-lateral head accelerations follow the same patterns of pelvis accelerations, however peaks are of smaller magnitude and, in the case of anteroposterior accelerations, they are not as clearly defined [71]. A plot of the signals obtained at the level of trunk and at the level of head is presented in Figure 2.22.

A single triaxial accelerometer attached at the lower back of trunk (Figure 2.23) has been frequently used (refer to [69], [45], [38], [31], [37], [75], [76] and [77]), since it produces the most reliable accelerometer signals. Actually, this structure is stable and near the COM of the body, moving parallel to it, which produces the same results for velocity, displacement and acceleration [36, 37]. Ideally, these movements present symmetry with respect to the anatomical planes [68].

Zijlstra and Hof (1997) has created an inverted pendulum model for the COM trajectory during walking, to predict the patterns of pelvic displacement, assuming that displacement patterns of COM and pelvis can be approximated by sinusoidal functions [68]. The changes that occur in the COM's height (vertical direction) depend on the step length. Thus, according to [69] the step length can be predicted using information from trunk acceleration signals.

\subsubsection{Gait parameters}

Several measures can be quantified during gait from accelerometer signal, which include gait velocity, cadence, step length, stride length, step time, stride time, swing time and 
Pelvis

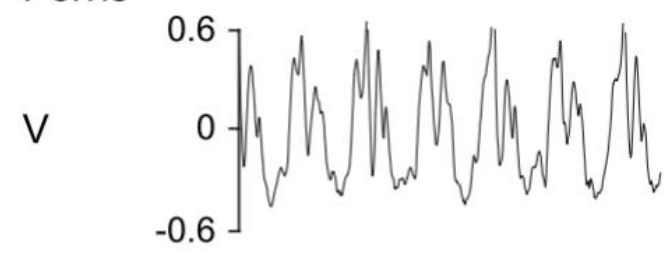

AP

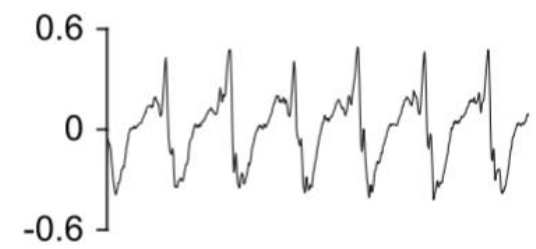

ML

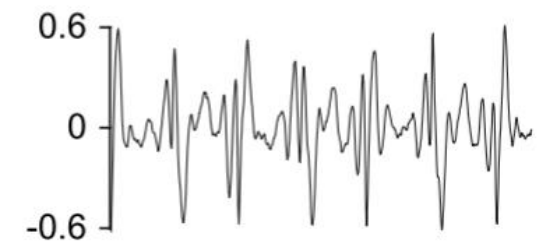

Head

V

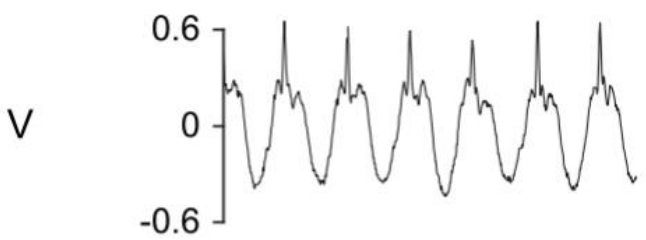

AP

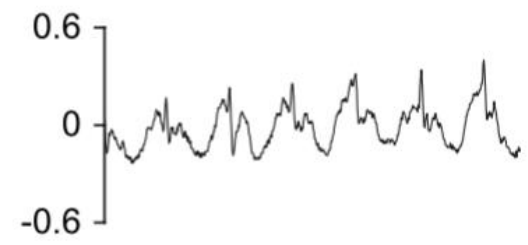

ML

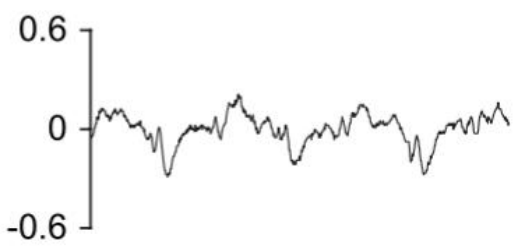

Figure 2.22: Pelvis and head acceleration signals. Adapted from [74].

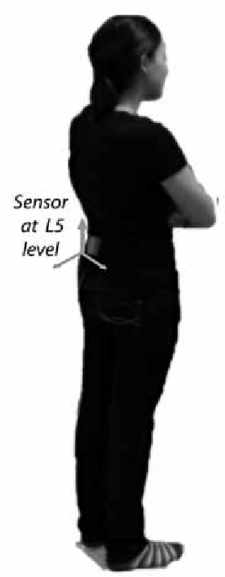

Figure 2.23: Lower trunk position of an inertial sensor. Source: [29].

double support time $[56,58,67]$, using the informations from peak detection algorithms [78].

Most of these parameters can be used to identify people with increased risk of future falls. However, for most of these features, cut-off values for clinical fall-risk assessment 
in older persons are lacking [31].

Verghese et al. (2009) concluded that slower gait speed (i.e. less than $70 \mathrm{~cm} / \mathrm{s}$ ) was associated with increased fall risk [56]. Brach et al. (2011) classified the individuals as having a poor/fair mobility (gait speed inferior to $80 \mathrm{~cm} / \mathrm{s}$ ), good mobility (gait speed between $80-100 \mathrm{~cm} / \mathrm{s}$ ) and very good mobility (gait speed superior to $100 \mathrm{~cm} / \mathrm{s}$ ) [57]. Data from [55] indicated that a slow gait velocity alone in well functioning older people was enough to predict risk for further adverse events. Then, the authors support the idea that the gait velocity test could be used in ambulatory clinics, possibly as an alternative tool to more complex mobility tests performed in clinic environments [55].

The study by Senden et al. (2010) also recognize the importance of velocity in the screening of fall risk [78]. The authors used a measure of normalized speed (i.e. normalized by person's height) to discriminate the gait of persons at risk and not at risk of falling. A normalized speed below 0.37 was indicative of a high risk of falling (sensitivity $87 \%$ and specificity $64 \%$ ).

However, some authors agree on the fact that gait velocity do not differentiate those who walk slowly but with a good pattern from those who walk slowly but with marked abnormalities [57].

Indeed, other studies found no association between reduced velocity and falling (cf. [79]).

Verghese et al. (2009) further identified that worse performance on swing, doublesupport phase, swing time variability and stride time variability were significant fall risk predictors [56].

Laufer et al. (2005) has found that increased forward gait speed is associated with a combined increase in both cadence and stride length [58].

The variability of gait parameters can be quantified by standard deviation (SD) [80, 81] or the coefficient of variation (CV) $[67,56]$, which is defined as (Equation 2.1):

$$
C V=(\text { standarddeviation } / \text { mean }) \times 100
$$

$\mathrm{SD}$ is a measure of how closely the individual data points cluster around the mean [82] and is defined by the following Equation 2.2:

$$
S D=\sqrt{\frac{\sum\left(X_{i}-M\right)^{2}}{N-1}}
$$

where $X_{i}$ is the value of each sample, $M$ is the mean value and $N$ is total number of samples [82].

A low stride-to-stride variability is related with efficient gait control and safety. A high stride time variability, in turn, is associated with future falls, and can be used to predict 
future falls [67]. Also, the older people with a high risk of falling present more variable step timing [83].

Brach et al. (2011) have found that older adults are less smooth in walking in the direction of motion than young adults [57]. The smoothness or regularity of walking is a measure of the rhythmic patterns of acceleration and deceleration of the trunk during walking. When a gait problem exist, a loss of the smooth forward progression of walking occurs [57].

The smoothness of walking can be quantified by the harmonic ratio calculated from trunk acceleration data, as concluded by [57]. The harmonic ratio is based on the frequency analysis of acceleration signals measured with a triaxial accelerometer attached at the lower back of the trunk, i.e. it is calculated as the power ratio of "in-phase" to "out-phase" components of the signal [57]. Higher ratios represent more stable walking patterns [74].

Gietzelt et al. (2009) have generated a decision tree to identify high risk of falling based solely on two parameters, extracted from the acceleration signals of lower back trunk (see Figure 2.24) [59]. These parameters were kinetic energy and pelvic sway. The kinetic energy was accessed by the following formula (Equation 2.3):

$$
E=(1 / 2) \cdot m \cdot v^{2}=(1 / 2) \cdot m \cdot\left(\int a \mathrm{~d} t\right)^{2}
$$

where $a$ is the magnitude length of the measured acceleration vector [59].

The pelvic sway was defined as the mean transversal acceleration deviation during walking [59].

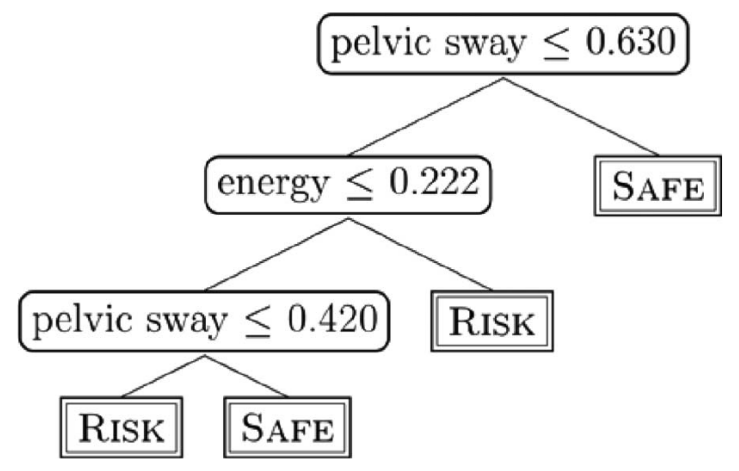

Figure 2.24: Decision Tree for fall risk prediction. Source: [59].

The study of Tura et al. (2010) has focused on the use of a single accelerometer to measure the gait symmetry and regularity, which are important parameters to consider for gait analysis [77]. The symmetry is related to the similarity of contra-lateral steps, whereas regularity is related to similarity of consecutive strides. Parameters were estimated from a generic unbiased autocorrelation function, Equation 2.4. 


$$
A d(m)=\frac{1}{N-|m|} \sum_{i=1}^{N-|m|} x(i) \cdot x(i+m)
$$

where $x(i)$ is the sample sequence, $N$ is the number of samples and $m$ is the time lag, expressed as number of samples.

They concluded that the first peak of $A d(m)$ along the anteroposterior axis, the $A d_{1}$ coefficient, could be a measure of symmetry between steps. The second peak of $\operatorname{Ad}(m)$ along the vertical axis, the $A d_{2}$ coefficient, should express the regularity between strides [77].

More detail is provided regarding the process of step length estimation, which is on the basis of other gait parameters determination.

\section{Step length}

Zijlstra and Hof (1997) conducted a study assuming that the trajectories of displacement of pelvis could be approximated by sinusoidal functions [68].

During walking, the vertical displacement can be modelled by a compass gait type during single support (i.e. approximated by an inverted pendulum model) and approximated by an inverted circular path during double support [68], as illustrated on the next figure (Figure 2.25).

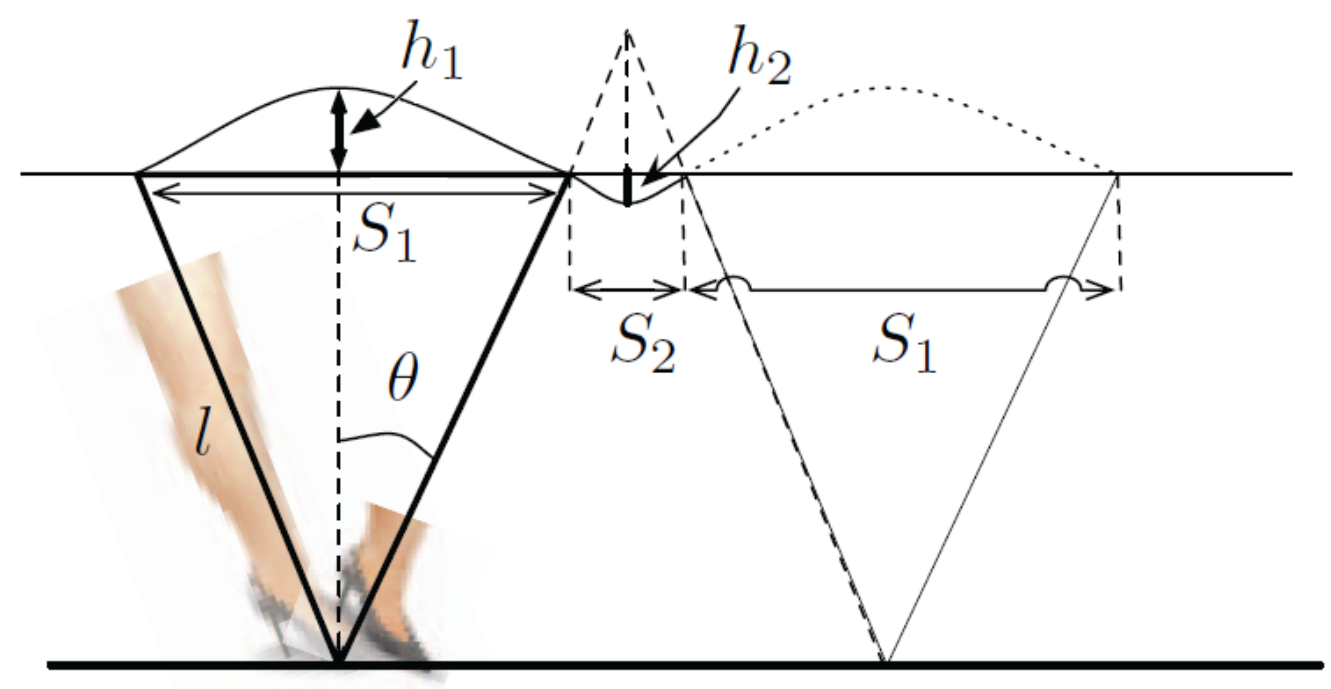

Figure 2.25: Model of the vertical displacement of the pelvis during walking. In this inverted pendulum model, the pendulum length $(l)$ is the leg length, $S_{1}$ represents the forward displacement during single support and $S_{2}$ the forward displacement during double support. Adapted from: [73].

According to [68], $h_{1}$, the vertical displacement during single support, can be calculated as Equation 2.5, using the geometrical relations shown in Figure 2.25. 


$$
h_{1}=l-l \cos \theta
$$

where $l$ is the leg length and $\theta$ is the angle between the vertical axis and the leg.

Using a trigonometrical identity $\left(\cos ^{2} \theta+\sin ^{2} \theta=1\right)$ and the relation from Equation 2.5, the following relation can be obtained, Equation 2.6.

$$
\sin \theta=\sqrt{\frac{2 h_{1}}{l}-\frac{h_{1}^{2}}{l^{2}}}
$$

The forward displacement of the pelvis during single support $\left(S_{1}\right)$ can also be calculated from geometrical relations, as in Equation 2.7.

$$
S_{1}=2 l \sin \theta
$$

Substituting Equation 2.6 in Equation 2.7, the following relation is obtained:

$$
S_{1}=2 \sqrt{2 h_{1} l-h_{1}^{2}}
$$

Zijlstra and Hof (2003) used this relation to predict step length based on changes on vertical position of COM [69]. The amplitude of changes in vertical position (represented as $h_{1}$ in Equation 2.8) was determined as the difference between highest and lowest position during a step cycle. In this study, all the step length measurements were underestimated for all the individuals and at all walking speeds.

In another study, Zijlstra (2004) used correction factors to improve the step length determination [45]. As the step length was underestimated on the previous study, this one used two different approaches to correct the step length determined by Equation 2.8. On the first approach, a standard multiplication factor (1.25) was used on all data. In the second approach individual correction factors calculated as the ratio between mean walking speed and predicted speed were used. The use of a standard multiplication factor improved the results, but still rough estimation of step length and walking speed was obtained. The use of individual correction factors further improved the results.

Later, in 2006, Alvarez et al. (2006) conducted a study where different step length estimators using wearable devices were compared [73]. The majority of the methods described included constants that required a calibration process, which had to be performed for each individual and was time consuming. Only one method was described that did not need any experimental calibration and could be applied using wearable devices. Since it produced similar results as the other methods, it was further explored and detailed.

The referred method was also based on an inverted pendulum model, as described above. However, the displacement of the pelvis was approximated by two pendulums: the first relative to the swing phase and described by the same relation as in Equation 
2.8, and the second relative to the double support phase, but with an unknown radius (see Figure 2.25). Total forward displacement during a step was then calculated as the sum of displacement during swing $\left(S_{1}\right)$ and displacement during double support phase $\left(S_{2}\right)$, as presented in Equation 2.9.

$$
\text { Steplength }=S_{1}+S_{2}=2 \sqrt{2 h_{1} l-h_{1}^{2}}+S_{2}
$$

The displacement $S_{1}$ is estimated from leg length $(l)$ and vertical displacement $\left(h_{1}\right)$ between the time of toe off and heel strike events. Displacement $S_{2}$ is set as a constant equal to the foot length [73].

All the methods described above require a common procedure, which is the double integration of the acceleration signal, in order to obtain the required position data. Zijlstra (2004) referred the use of a 4th order zero-lag Butterworth filter in order to avoid integration drift on position data [45]. Alvarez et al. (2006) refers a more tricky process in order to avoid drift, which is to force the final values of the integrals to be the desired ones, having in mind that at the time of foot-flat the velocity (i.e. the result from the first integration) is zero and vertical coordinate of the COM (i.e. after second integration) is the same that at the beginning of the step [73].

Integration processes will be further explored on the next paragraphs.

\section{Integration}

As is known, the acceleration can be calculated as the first derivative of velocity or the second derivative of position. It is here of interest to reverse this process in order to obtain velocity and position from the acceleration data, which is extracted from sensors. This can be achieved by integration of signals [84].

A common way to integrate a digital signal (e.g. the acceleration obtained from sensors) is using the Trapezoidal Rule [85, 86], Equation 2.10.

$$
i(n)=i(n-1)+\frac{1}{2}[x(n)-x(n-1)][y(n-1)+y(n)], \quad n>0
$$

Integrating a signal is a quite simple process. However, there are some problems that must be addressed before performing double integration.

Firstly, initial conditions are required by integration formula, i.e. velocity and displacement at the start of the measurement. When referring to integration of acceleration signal this can be a problem, since initial conditions are not known.

Another problem that has to be considered is the cumulative characteristic of integration, meaning that any drift caused by small mean value variations in the acceleration signal can lead to large integration errors, which are translated in a time-increasing error. This problem is even magnified when the signal is double integrated. Signals from a 
real accelerometer can even become unbounded over time, which is basically due to low frequency components of the signal [84, 85].

A mathematical explanation of these problems is provided hereinafter.

If the acceleration signal is composed by a zero-mean time-varying component of interest $(a(t))$ and a constant representing drift $\left(d_{0}\right)$, it can be represented by the following equation:

$$
A(t)=a(t)+d_{0}
$$

The integration of this signal, assuming zero initial conditions, results in Equation 2.12 .

$$
V(t)=\int_{0}^{t} A(\tau) d \tau=\int_{0}^{t} a(\tau) d \tau+\int_{0}^{t} d_{0} d \tau=v(t)+d_{0} t
$$

where $V(t)$ is the composite velocity signal and $\mathrm{v}(\mathrm{t})$ is the desired velocity. This first integration results on the appearance of a component causing a drift, $d_{0} t$, which is a ramp with a slope of $d_{0}$. The second integration of this signal, again considering zero initial conditions, will result on the following equation (Equation 2.13).

$$
X(t)=\int_{0}^{t} V(\tau) d \tau=\int_{0}^{t} v(\tau) d \tau+\int_{0}^{t} d_{0} \tau d \tau=x(t)+\frac{1}{2} d_{0} t^{2}
$$

where $x(t)$ is the desired component of position signal and $\frac{1}{2} d_{0} t^{2}$ is an unwanted quadratic component.

As can be observed through the analysis of the last equations, an accelerometer drift can result in large integration errors, which can become unbounded over time. To eliminate this problem, acceleration drift must be eliminated. This can be achieved by applying a high-pass filter before the integrating the signal, which will remove the mean time-varying component of signal [85].

The second problem, which is the lack of initial conditions in an experimental situation, will also be a source of errors, which should be eliminated. This problem can be illustrated be the following set of equations.

Supposing that initial velocity is known $\left(v_{0}\right)$, the first integration of acceleration signal can be represented by Equation 2.14.

$$
v(t)=\int_{0}^{t} a(\alpha) d \alpha+v_{0}
$$

Position can be obtained by integrating velocity, which, assuming $x_{0}$ as initial condition, can be represented by Equation 2.15.

$$
x(t)=\int_{0}^{t} v(\alpha) d \alpha+x_{0}
$$


Substituting Equation 2.14 on Equation 2.15 the following result is obtained.

$$
x(t)=\int_{0}^{t} \int_{0}^{\tau} a(\alpha) d \alpha d \tau+v_{0} t+x_{0}
$$

The lack of initial conditions will then cause a linear increase of output after the second integration $\left(v_{0} t\right)$ and also a constant $\left(x_{0}\right)$ added to the zero mean time varying signal.

A solution to eliminate the need of initial conditions is to use filtering. After the integration of acceleration, a high-pass filtering of signal will remove the mean varying component. On the same manner, after integrating velocity to get position, the signal must also be high-pass filtered.

Then, the double integration step must start with high-pass filtering to eliminate the accelerometer drift. Then, after integration, velocity is found and another filtering must be done in order to eliminate the need of initial condition. The second integration will result on position, which must also be high-pass filtered in order to eliminate low frequencies and the need for an initial position measurement [85]. The steps involved on the double integration process are presented in Figure 2.26.

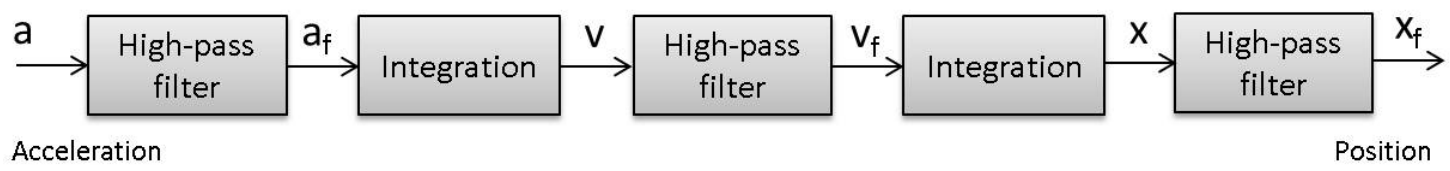

Figure 2.26: Block Diagram of Double Integration Process.

The high-pass filters to be used on the double integration process will pass the high frequencies while rejecting the low frequencies. A proper specification of filters is necessary, regarding its frequency response, filter order and delay. There are different filter types which can be used: FIR (Finite Impulse Response) filtering, IIR (Infinite impulse response) filtering and FFT (Fast Fourier Transform) filtering. A complete discussion about their advantages and disadvantages is provided by [85].

FIR filter has a linear phase response, which means that different frequencies will have the same time delay. A disadvantage about using this filter is that high order of filter must be necessary, since a low cutoff frequency will be needed. This means that a large delay from input to output will be observed (delay will be equal to the order of the filter divided by two) and each stage of filtering will accumulate large time delays, resulting in a loss of a significant amount of data [85].

The IIR filter (e.g. Butterworth filter or Bessel filter) has a lower order than the corresponding FIR filter. However, its phase response is not linear, meaning that different frequencies will be delayed differently. Some techniques exist to linearise phase response of filter [85]. 
A different alternative to these two conventional filter types is also proposed, which is the FFT filtering. Using this technique, a FFT of signal is necessary and then the low frequencies near mean varying component are removed. To do this, the lower frequency coefficients are modified and then an inverse FFT retaining only the real part of signal enables its reconstruction to obtain a filtered signal [85].

The lower frequency coefficients are located at the beginning and end of the FFT sequence. The coefficients at the end must be the conjugated of the ones at the beginning, because FFT sequence must be conjugate symmetric so that the signal remains real. Low frequency coefficients must be zeroed, so that low frequency components are removed. FFT filtering algorithm can then be written as:

$$
\begin{aligned}
& X=f f t(x) ; \\
& X_{f}=X ; \\
& X_{f}(0)=\alpha_{0} X_{f}(k) ; \\
& \text { for } i=1:(k-1) \\
& X_{f}(i)=\alpha_{i} X_{f}(k) ; X_{f}(N-i)=\operatorname{conj}\left(X_{f}(i)\right) ; \\
& x_{f}=\operatorname{Real}\left(\operatorname{IFFT}\left(X_{f}\right)\right) ;
\end{aligned}
$$

where $x$ is the input signal, $x_{f}$ is the filtered signal, $k$ is the index of FFT coefficient representing the cutoff frequency and $\alpha_{i}$ 's are the coefficients specified by the user [85].

\subsection{Smartphones and Android}

Modern mobile phones are not just simple communication devices. They have now the ability to detect physical and environmental properties, such as image, acceleration, orientation, light, magnetic field and temperature, offering new possibilities to enhance mobile applications. These abilities are possible through the use of several sensors, such as accelerometers, compasses, gravity and brightness sensors [12].

An Android based phone runs the Android operating system. Android refers to an open-source operating system and to an open-source development platform, which gives anyone the opportunity to create mobile applications.

The Android software development kit (SDK) includes everything that is necessary to develop, test and debug applications. This includes the Android API libraries, development tools (to compile and debug applications), the Android emulator (that simulates the device hardware configuration), full documentation, sample code and online support. The Android code is written in Java syntax [12, 87].

Android also offers a plug-in for Eclipse, called Android Developer Tool (ADT) plugin. Eclipse is an open-source IDE (integrated development environment) that is particularly popular for Java development. The plug-in simplifies the Android project creation, testing, and debugging, by integrating the developer tools and emulator into the IDE $[12,87]$. 
The API libraries simplify development involving a device hardware, in particular, Android abstracts the sensor implementation of each device. Each hardware sensor is described on the Sensor class and different type of sensors are available on the smartphone. The Sensor class is used to describe the properties of each hardware sensor, including its type, name, manufacturer and details on its accuracy and range [12].

The supported sensor types are:

- Accelerometer: Measures the current acceleration along $x$ (lateral), $y$ (vertical) and $z$ (longitudinal) axes relative to the screen of the phone (as represented on Figure 2.27) in SI (International System) units, i.e. $\mathrm{m} / \mathrm{s}^{2}$. Acceleration is defined as the rate of change of velocity. Accelerometers measure both static (e.g. gravity) and dynamic (e.g. vibration) acceleration, being unable to differentiate between acceleration caused by movement or caused by gravity $[12,33,88]$.

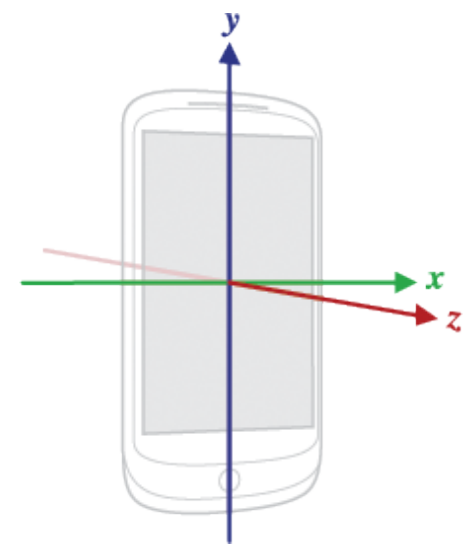

Figure 2.27: Acceleration sensor coordinate system. Source: [87].

- Gyroscope: Measures the rate of rotation around the same three axes on Figure 2.27, in radians/second. The rotation is positive in the counter-clockwise direction. The calculation of an angle is done by integrating the output of gyroscope over time [87].

- Light: Tells how bright is the surrounding area, in lux.

- Magnetic field: Measures the current magnetic field along the three axes, in microTesla.

- Pressure: Senses the current atmospheric pressure, in kilopascals.

- Proximity: Provides the distance between sensor and some object, in meters.

- Temperature: Returns the temperature of the surrounding area in degrees Celsius $[12,88]$. 
The accelerometers and compasses can be used to determine device direction, orientation and movement [12].

More recently, some smartphones exists already incorporating a fusion of sensors, i.e. a combination of sensory data in order to produce enhanced data [89]. This enable the direct extraction of several new sensor reading types, such as the linear acceleration and gravity. This can then be used for the development of applications that quickly and smoothly respond to precise changes in device position and motion [87].

Linear acceleration is the result from eliminating the force of gravity from real acceleration signal of the device. The isolation of the force of gravity is achieved through the application of a low-pass filter on the real acceleration signal. On the contrary, linear acceleration can be obtained by applying a high-pass filter [87]. Accelerometer, gravity and linear acceleration (all values have units of $\mathrm{m} / \mathrm{s}^{2}$ ) then obey to the following relation 2.17:

$$
\text { acceleration }=\text { gravity }+ \text { linearAcceleration }
$$

\subsection{Mobile phones and older people}

Mobile devices are small, have small screens and buttons, so that older people can have difficulties operating them. Often, they are also unfamiliar with the interaction techniques used on mobile phones [90] and experience fear of consequences of using them [91].

For that reason, the effectively use of devices by older persons can only be achieved if interfaces are carefully designed [90], since they prefer design features that aid them due to their declining functional abilities [91].

The interface design for older people is more complex than for younger groups, since older persons experience a slower processing of spatial, verbal and visual information and have frequently a loss of memory [92]. Also, as the effects of ageing don't manifest at the same rate for everyone, the variability increases as the persons grow older, which presents more challenges to interface design [93].

Interfaces should then be:

- easy to use;

- simple;

- intuitive, so that the users are not forced to relearn the application every time they use it;

- functional;

- support a proper interaction between finger and the screen [12]. 
Background and Literature Review

According with [90] the currently available design guidelines are based on studies with younger users and may not properly apply to applications directed to older people. 
Background and Literature Review 


\section{Chapter 3}

\section{Fall Risk Screening Method}

Based on current state of the art, fall risk prediction can be done using different tools and techniques. At this project the phone is suggested as a potential instrument for these purposes.

Since it has to be manipulated autonomously, there are some requirements that need to be accomplished, regarding the person's safety and the easiness to understand and perform the tests.

In this chapter, the hypothesis and projects considered to design a fall risk screening method are presented. Then, a detailed explanation of adopted methods is provided.

\subsection{Hypothesis and projects}

According with recent recommendations, the fall risk screening must be based in two main factors, which are the history of falling and gait and/or balance impairments. Older persons (i.e. people aged 65 years and older) should be screened for fall risk frequently, so that preventive measures can be designed before the occurrence of a fall (see Chapter 2, Section 2.4).

Clinically, some ordinal scales (such as BBS, POMA and BESTest) are being used, requiring an observer to rate the person's ability to perform some tasks. Consequently, these scales result in some subjective results [7, 31, 32].

Other functional performance tests, such as TUGT, FRT or Turn $180^{\circ}$, are based on the measurement of some simple outcomes, e.g. the time to complete the task or the number of steps required to perform it. Usually, some observations considerings are also taken into account. 
As already mentioned (see Chapter 2, Section 2.5), the instrumented assessments are becoming increasingly important, since highly accurate results are obtained for a quantitative evaluation of gait and balance. In particular, inertial sensors are becoming an alternative to the traditional methods, such as force-plates and optical motion systems. They offer several advantages when comparing to traditional methods, for example, they are portable, cheaper and lighter. Authors also recognize that the use of such systems can address the problems related with the low frequency of fall prevention strategies application, i.e. through the application of risk screening techniques in the community [11].

The smartphone has the advantage of being equipped with inertial sensors, such as accelerometer and gyroscope (see Section 2.10 of Chapter 2). In this context, the smartphone could be used for fall risk screening purposes.

Since unsupervised assessments are expected, the selection of movements must be performed carefully, so that the person's safety is guaranteed. Also, the tests must be easy to understand.

On Section 2.7, several studies using inertial sensors to evaluate balance and gait were reviewed. All the studies had in common the use of inertial sensors while performing the movements of functional performance scales, such as TUGT, SiSt and StSi transfers or simply walking. Frequently more than one sensor attached at different places of the body were used.

As the phone is equipped with inertial sensors that are confined at one place, only the studies using solely one place of attachment could be analysed in depth. Particularly, the studies focused on gait analysis used only an accelerometer that was attached at the lower back of trunk (see Section 2.9 of Chapter 2).

The acceleration signals obtained on the trunk were described in detail by different authors and associations between some acceleration-based gait parameters and an increased fall risk was reported, so that these measures can be used to discriminate high and low risk groups.

Then, the risk screening process could be done through the analysis of the data obtained from phone's inertial sensors during gait. As walking is the way people move, it satisfies the requirements of safety previously established, and can be easily performed by the older person.

The phone can then be used to screen the risk of falling over extended periods of time. In addition, some risk factors for falling (i.e. those related with the self perceived risk) can be assessed by some questionnaires and a risk profile can be built. These results can be recorded with an associated time, so that an history of risk factors can be built and better strategies of prevention can be designed/modified by the doctor. This can be done with a view to calculate a time-dependent likelihood of falling, based on several risks factors, as supported by many articles (Section 2.7 , Chapter 2). 


\subsection{Inertial sensors signal recording}

An Android based phone was used to record sensors data. The phone has Android version 2.3, i.e. API level 9 [87], including sensor fusion APIs (i.e. rotation matrix, linear acceleration and gravity) and is equipped with a three-axis gyroscope, accelerometer, digital compass, proximity sensor and light sensor [94].

The process to record data from sensors is quite simple in Android and is completely described in [87] and [12]. Eclipse with ADT plug-in was used to develop a simple program capable of measuring and recording the data from sensors.

Activity is the base class of the application. When creating an Activity-based class it extends Activity and override the oncreate method.

The Sensor Manager class is used to manage the hardware available on the phone. The first step is then to return a reference for Sensor Manager Service, as presented in the next line code, which is done inside oncreate method.

sensorManager $=($ SensorManager $)$ getSystemService $($ Context.SENSOR_SERVICE $)$;

Then, an implementation of the SensorEventListener is registered with the Sensor Manager, using a Sensor object of a specified type to request for its updates:

sensorManager. registerListener ( mySensorEventListener, accelerometer,

SensorManager.SENSOR_DELAY_FASTEST);

When creating the SensorEvent Listener the onSensorChanged method (which is used to monitor the Sensor values) and the onAccuracychanged method (which reacts to changes on Sensor's accuracy) are override, as shown on the next code lines.

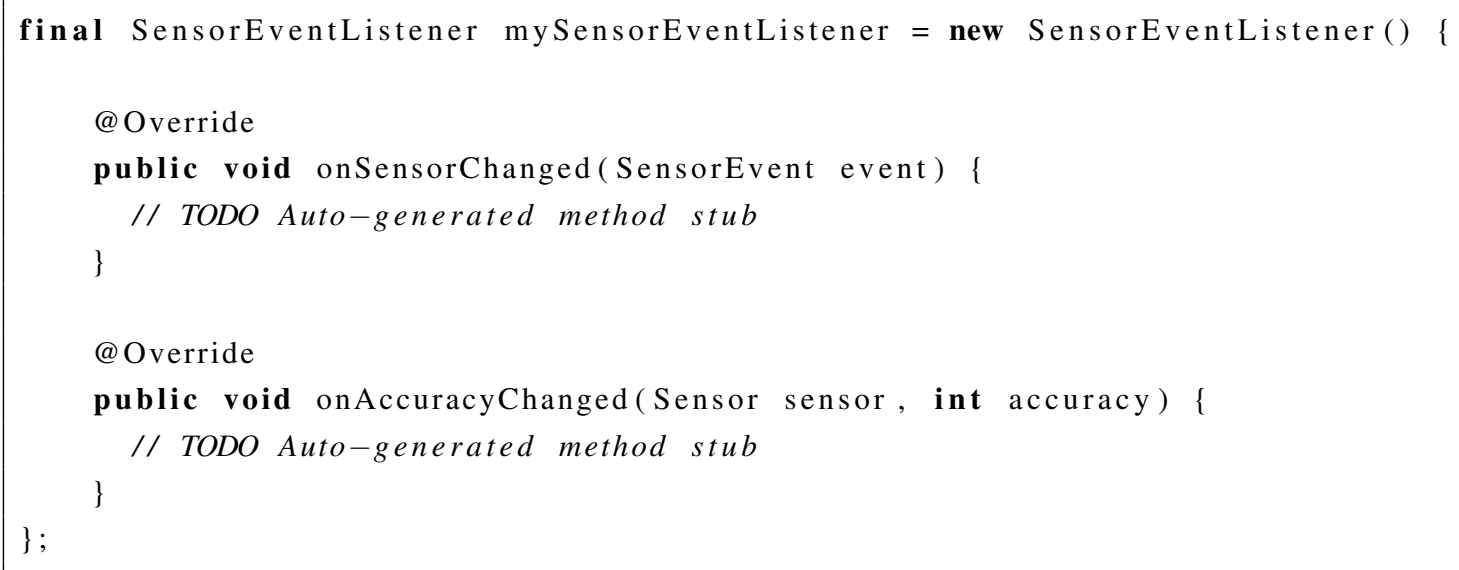

When the application no longer needs to receive Sensor updates, the Sensor Event Listeners are unregistered in onResume and onPause methods, ensuring that they are only being used when the Activity is active [12].

Time of each sensor reading is obtained using system time, i.e using currentTimeMillis() method. 
Each triaxial sensor data and the time of each sample recording is stored on the SD card (i.e. the external storage) in a text file. This is done using streams, as described by [87]. The text file name include the sensor type, the name of the person being tested and the time when data was stored.

All the data from all the available sensors or sensor fusion are recorded (i.e. acceleration, gravity, gyroscope, light, linear acceleration, magnetic field, orientation, proximity and rotation), so that an analysis of each signal and the possibilities of using them could be evaluated.

\subsection{Data Analysis and Events Detection}

The data recorded using inertial sensors is transferred from the device to the computer, so that it can be analysed. This was done using the Dalvik Debug Monitor Server (DDMS), which is integrated into Eclipse. DDMS is a debugging tool that, among other services, provide a File Explorer tab, that enables to view, copy and delete files on the device [87].

Then, MatLab ${ }^{\circledR}$ was used to analyse data and compute the gait parameters identified on the literature. MatLab ${ }^{\circledR}$ is a high-level language with an interactive environment for managing code, files and data. It includes several mathematical functions for linear algebra, statistics, Fourier analysis, filtering and numerical integration, being widely used for signal processing purposes. Graphics functions are also available to easily visualize data [95].

On the following paragraphs, the methods involved on signal analysis and detection of signal events are detailed.

\subsubsection{Trial tests recording}

For the method's development, some trial tests were recorded, using phone sensors. These tests simply consisted in walking straightforward, at different speeds, with the phone attached at the lower back of the trunk using a belt (as shown in Figure 2.23).

Phone was always positioned with the screen oriented to the back and its top to the right. Its axis were then on the position represented in Figure 3.1, i.e. $z$ axis backward, $x$ axis downward and $y$ axis to the right. To be consistent with the International Society of Biomechanics (ISB) recommendations [96], the signal of each recorded data in each axis was adjusted, so that axis were oriented upwards, forward and to the right of the direction of progression (see Figure 3.1).

For the recording and storage of signals, an application already developed at Fraunhofer AICOS was used. With this application, sensors signal recording is performed during a time frame, which is 10 seconds. Also, a delay of 5 seconds is provided before starting to record, which enables the phone to be positioned at the appropriate place and 

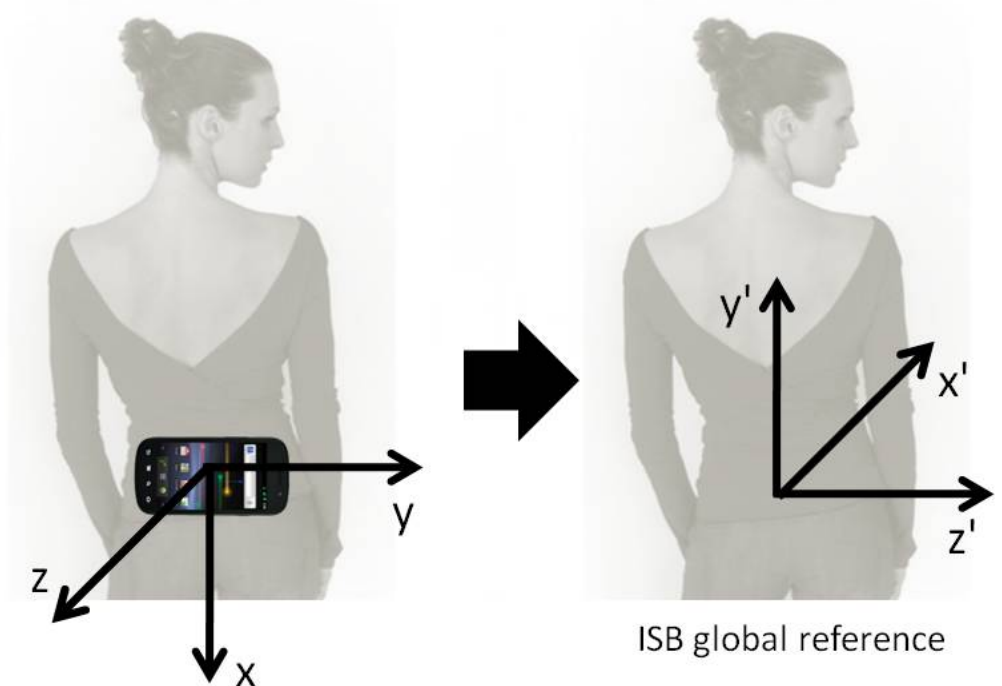

Figure 3.1: Phone coordinate system and ISB conventions in the reporting of kinematic data [96].

stabilize it before the recording begins. An audio beep informs the user about the moment when recording starts and finishes. Then, trial tests were recorded by walking until the audio signal indicating the end of recording is listened.

To study the moment when walking is started and having in mind that this test is being developed to be performed without assistance, some trial tests were recorded beginning with quiet standing and then starting to walk.

The data from these trial tests was used for the development of a gait analysis algorithm in MatLab ${ }^{\circledR}$.

\subsubsection{Foot contacts detection}

After reading the sensors signal data, foot contacts detection is done. The correct detection of these instants will be fundamental for a reliable determination of other essential spatio-temporal factors, which are very dependent of this phase [69].

Foot contacts are defined as the peaks forward acceleration preceding a change of sign (from positive to negative), as described earlier in Section 2.9 and shown in Figure 2.19. However, this simple definition was not enough for a proper detection of foot contacts in real signals, because not every acceleration peaks obeying to this rule corresponded to foot contacts, as can even be observed on the theoretical signal of Figure 2.19. So, other characteristics of signal were taken into consideration.

To follow the methods presented on the literature, acceleration signal was used. As linear acceleration presented similar characteristics, it was also considered on the method development. Acceleration and linear acceleration signals do not presented much noise 
(based on the theoretical signals presented on the literature), so that no pre-processing was done before starting to detect foot contacts.

So, the first step was to detect the zero crossings on the forward component of the linear acceleration signal. Linear acceleration was selected for this purpose because it was already adjusted around zero, presenting a more regular pattern around this value, so that the process of detecting zero crossings was facilitated. Zero crossings from positive to negative were defined as two consecutive samples defining a line with negative slope and whose product resulted into a negative or zeroed value. For each zero crossing, the preceding maximum was calculated. This was performed looking on the preceding samples, and a maximum was considered when a previous sample was lower that the actual one. A schematic graph is provided on Figure 3.2 to better visualize and understand the process of zero crossings and maximum detections.

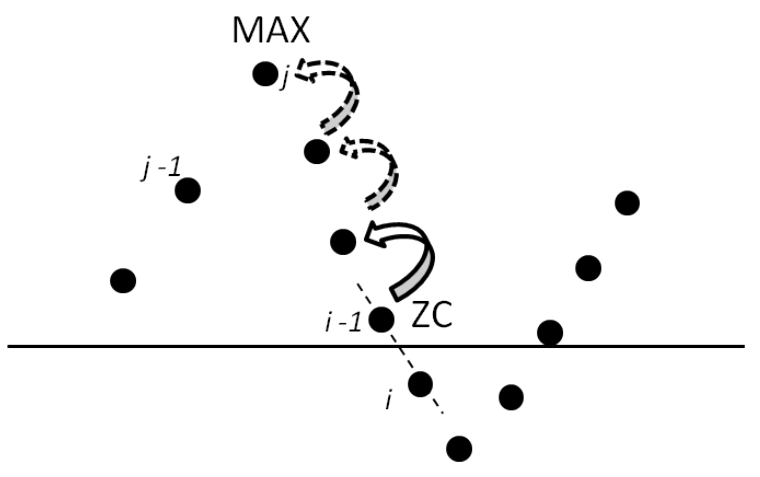

Figure 3.2: Zero Crossings (ZC) and maximums (MAX) detection.

To exclude the zero crossings due to signal noise, only the ones with a preceding maximum above a given threshold were considered. This parameter was fixed at $0.5 \mathrm{~m} / \mathrm{s}^{2}$, based on a set of walking tests. This discrimination was still performed on the linear acceleration signal, because as the signal is around zero the fixation of a parameter can easily be done.

Then, the forward acceleration maximums preceding the selected zero crossings instants were conserved as potential heel contacts, as described on the literature (i.e. cf. [45]).

As some peaks were detected on the quiet standing phase (i.e. on the first parts of signal), a process was developed in order to eliminate those detections. To this purpose, a measure of a sliding standard deviation (Equation 2.2) of forward acceleration was used. In MatLab ${ }^{\circledR}$, the function STD is used.

It was considered that walking started after more variability is observed relative to the quiet standing phase. The standard deviation was calculated inside windows with a fixed length (10 samples) along all acceleration signal (as illustrated on Figure 3.3). Then, the 
mean value of standard deviation was calculated. The moment when walking started was considered when standard deviation started increasing, reaching a value greater than its mean. All the maximums before this moment were excluded, as they were part of standing phase.

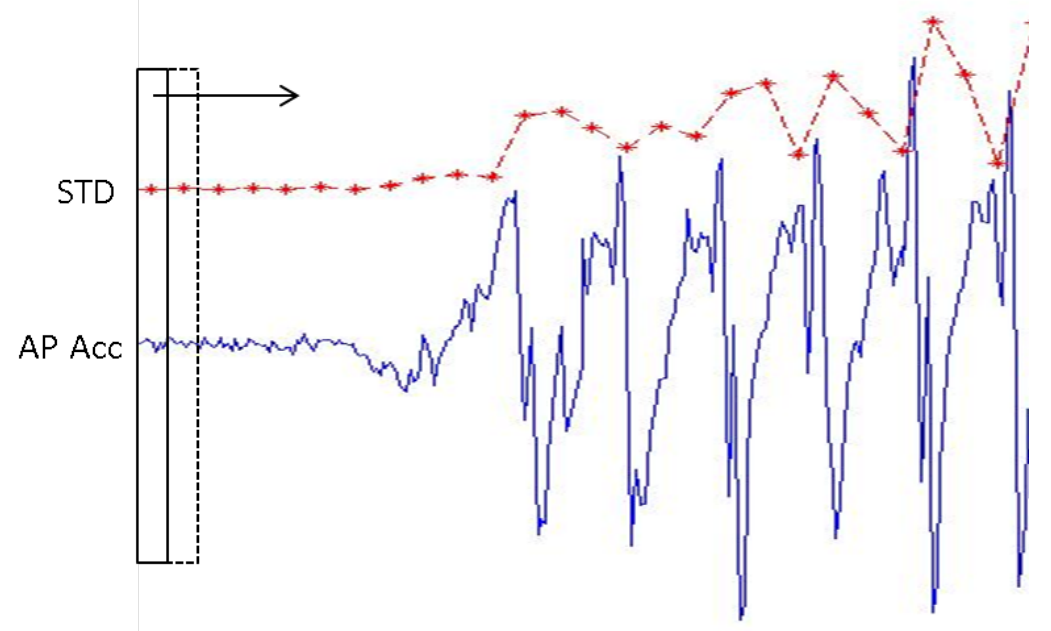

Figure 3.3: Sliding standard deviation illustration. The window has a fixed length of 10 samples. Red: standard deviation (STD); Blue: Forward acceleration signal (AP Acc).

After this process, some maximums not representing heel contacts were still conserved. To eliminate those peaks, a mean difference between the times of two consecutive peaks was calculated in order to estimate which of them were too close, considering that walking has a quite regular pattern and a regular frequency of heel contacts. Peaks were considered too close if the time difference between them was less $80 \%$ of the mean, admitting that some variability may be present on the walking pattern. This value was determined experimentally using the trial tests. On those situations only the higher peaks were conserved, expecting that they corresponded to foot contacts.

\subsubsection{Right vs. Left foot contacts discrimination}

After heel contacts detection, discrimination between right and left heel contacts was performed. It was based on the medio-lateral linear accelerations of the trunk.

Menz et al. (2003) stated that shortly after a heel contact, a rapid acceleration on the contralateral direction is observed on the medio-lateral component of acceleration [71].

As recognized by Zijlstra and Hof (2003), the major part of the medio-lateral acceleration is to the left during the right support phase, and vice versa [69]. So, the prevalence of positive accelerations (i.e. accelerations to the right) after a heel contact was considered a sign of a left support phase, and vice versa. Linear acceleration was used instead 
of acceleration, because it was adjusted around zero, and peaks were more pronounced. Since the medio-lateral acceleration signal is weaker than the others, because it has quite sporadic parts of signal $[71,72]$, sometimes this rule did not apply.

To improve the results, a Butterworth low pass filter (order 1 and cutoff $10 \mathrm{~Hz}$ ) was applied to attenuate some of those random parts of signal. This filter belongs to the IIR filtering type (as referred previously on Section 2.9). As discussed earlier, this filter has a lower order than the corresponding FIR filter and some techniques exist to linearise its phase response. MatLab ${ }^{\circledR}$ has already this functions implemented, so that application of filter is facilitated. The butter $\left(\mathrm{N}, \mathrm{Wn},{ }^{\prime}\right.$ l low' $)$ function designs a Nth order low pass filter, with a cutoff frequency $W n$, returning the filter coefficients. Then, filtfilt function performs a zero-phase forward and reverse digital filtering, so that the phase response of filter is linearised [85].

A low order of filter was selected, because when applying the linearisation technique, the order of filter is effectively doubled [85]. The cutoff frequency was set experimentally and based on data from literature, allowing to preserve the stride frequency (i.e. the periodicity of medio-lateral linear acceleration signals) and eliminating higher frequencies of signal. According to [69], stride durations may vary from $0.98 s$ to $1,74 s$ when walking velocity varies from $1.75 \mathrm{~m} / \mathrm{s}$ to $0.50 \mathrm{~m} / \mathrm{s}$. These stride durations can then be considered the period of the medio-lateral signal (the signal repeats after a complete stride), so that fundamental frequencies may vary from $0.57 \mathrm{~Hz}$ to $1.02 \mathrm{~Hz}$. Then, the frequencies of steps are doubled. A cutoff of $10 \mathrm{~Hz}$ enables a very smooth filtering of signal, conserving its main characteristics (i.e. frequencies corresponding to strides and steps).

After filtering the signal, a sum of medio-lateral linear acceleration values between foot contacts was done, to evaluate if a prevalence of positive or negative values were encountered.

Then, taking into consideration that an alternate pattern between right and left foot contacts is expected to be observed, the "left vs. right" classification was done according to the pattern (left-right or right-left) that predominated. So, only two predicted patterns could be present: beginning with a right contact or beginning with a left contact. A score was calculated for each pattern, summing one when the performed detection was in agreement with that pattern. The pattern with higher score was used to classify foot contacts, expecting that true detections were more frequent than false detections.

\subsubsection{Toe offs detection}

Toe offs were detected on the vertical linear acceleration signal. Once again, this signal was used because it was already adjusted to eliminate the static component of vertical acceleration, i.e. the gravity. 
The minimums after heel strikes were considered the toe offs [73]. To detect these instants, the samples after a foot contact were evaluated. A sample represented a minimum if the previous and next samples were larger than the actual one (see the illustration of the process in Figure 3.4).

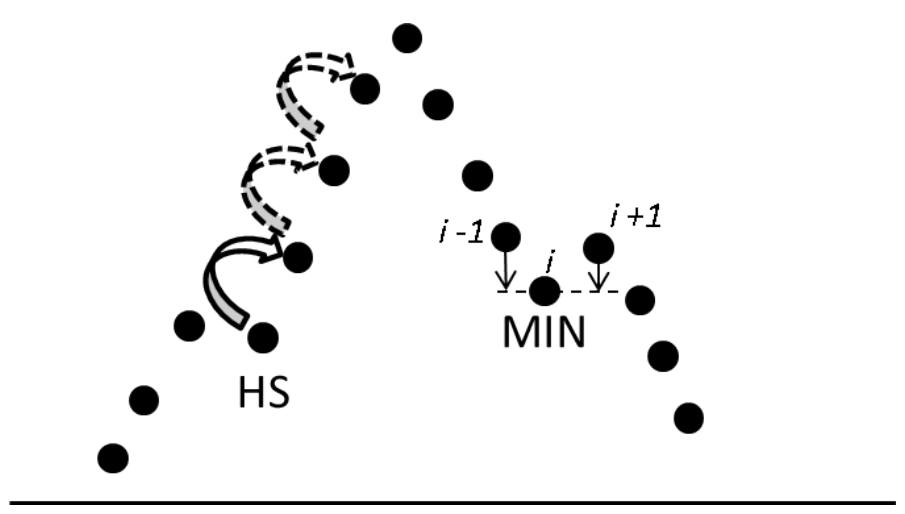

Figure 3.4: Illustration of a toe off detection. HS: heel strike; MIN: minimum representing toe off.

Discrimination between left and right toe off was determined based on the previous information about right and left foot contacts. After a left foot contact, a right toe off is expected and vice versa.

\subsubsection{Gait Phases}

Right and left foot contacts and toe offs information was enough to delimit gait phases.

It was possible to evaluate its phases, regarding events time, from a left side perspective and from a right side perspective (see Figure 3.5).

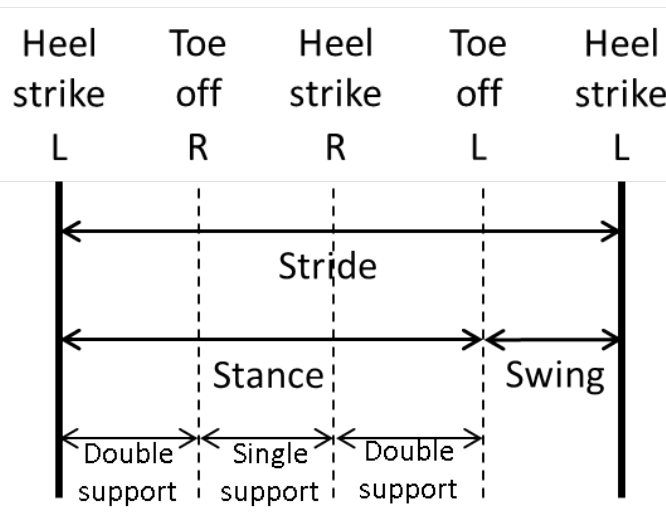

(a) Left side

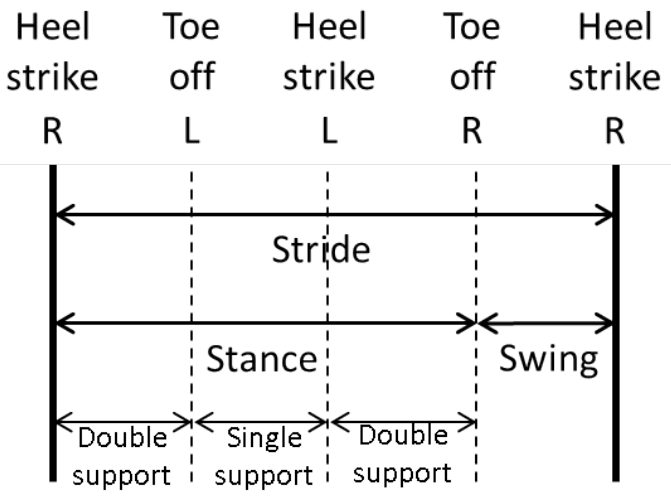

(b) Right side

Figure 3.5: Gait phases from right and left side. 
A stride was considered the time between two consecutive heel strikes of the same side. A left stride was delimited by left foot contacts (Figure 3.5a) and a right stride by right foot contacts (Figure 3.5b).

The stance phases were considered the time between a heel strike and a toe off of the same side, i.e. left stance and right stance phases could be identified. Swing phase began after a toe off and finished after a heel contact of the same side.

During stance, single and double support phases were determined. Double supports happened between heel strike and toe off of the other foot and single support between toe off and heel strike of the same foot (Figure 3.5).

\subsubsection{Steps duration}

Steps duration were calculated as the time between two consecutive foot contacts (cf. [69] and [45]).

Left steps and right steps duration was also calculated. A left step duration was calculated from the time between a right foot contact and a left foot contact. Right steps duration were calculated as the time between a left foot contact and a right foot contact.

Mean steps duration was calculated, as well as mean left steps duration and mean right steps duration.

\subsubsection{Step length estimation}

The estimation of step length was based on an inverted pendulum model using the Equation 2.9, as described earlier on Section 2.9 of Chapter 2.

To this process, it was necessary to double integrate the vertical acceleration signal, in order to obtain the vertical displacement of the pelvis. As described on Section 2.9, integration of these kind of signals presents several problems, which were solved by some high pass filtering of signal. The block diagram of this process was described in Figure 2.26 and the FFT filtering algorithm was also presented.

So, the vertical acceleration signal was first filtered using FFT filtering. This type of filtering is quite simple to perform using MatLab ${ }^{\circledR}$. The $\mathrm{fft}$ function enables the calculation of the discrete FFT transform of acceleration signal. Then, this spectrum is modified, so that lower coefficients located at the beginning (i.e. below the cuttoff frequency) and the end of the spectrum are zeroed. Coefficients at the end are the conjugated of the ones at beginning. The cutoff was determined based on the signal characteristics, which was the frequency of heel strike contacts (that was determined from the mean step duration value). This is the fundamental frequency of vertical acceleration signal, since its periodicity is equal to step duration. The ifft function enables the reconstruction of the signal from the modified FFT spectrum, by an inverse FFT. As the measured acceleration is a 
real signal, only the real part of the reconstructed signal was conserved. To do this, real function was used.

The filtered vertical acceleration is then firstly integrated to obtain the velocity signal. Integration was performed using the Trapezoidal rule (as described in Section 2.9, Equation 2.10).

Velocity signal was again high pass filtered, using the same cutoff frequency and multiplication factor. After reconstruction, signal was integrated again, so that a position signal could be obtained.

This signal was again high pass filtered and reconstructed using the same process.

To estimate the step length of all available steps on the overall signal, the vertical displacement between toe off and heel strike of the same leg was calculated. The amplitude of changes in vertical position between these instants was calculated as the difference between highest (i.e. maximum) and lowest (i.e. minimum) position, as described previously on Section 2.9.

Step length estimative was calculated as the sum of the displacement during swing and the displacement during double stance. Displacement during swing was calculated from the vertical displacement and from the leg length. Displacement during double stance was approximated by foot length (see Equation 2.9).

Mean step length was calculated from all available steps in a trial.

\subsubsection{Walking speed and variability parameters}

Walking speed was calculated by simply dividing the mean step length by mean step duration (cf. [69]). A normalized speed was also calculated, dividing walking speed by the height of the person (cf. [78]).

Based on the literature review, several variability parameters were also calculated, since they were identified as having a relation with the risk of falling (see Section 2.9). They can be calculated using standard deviation, or using a coefficient of variation (cf. [80] or [81]), as described previously on Section 2.9.

Then, standard deviation and coefficients of variation were calculated to quantify stride time, swing time, double support time and stance time variability.

The mean percentage of swing time, stance time and double support time, relative to each corresponding stride duration, were also calculated (cf. [80]). These measures represented the mean percentage of stride time spent in each gait phase, i.e. swing, stance and double support.

The standard deviation and a coefficient of variation were also calculated for each stance time percent (i.e. relative to each corresponding stride) (cf. [80]).

Other measures were also calculated based on the description provided in [59], i.e. pelvic sway and kinetic energy. They were calculated as described previously on Section 
2.9. However, a low confidence exist on the calculated values, since the description that is provided on the article is not very clear and a report on the expected values is also not provided. These measures were then not furthered considered.

\subsection{Evaluation method}

\section{Participants}

A group of 14 participants (mean age $26 \pm 3.6$, height $1.74 \pm 0.1 \mathrm{~m}$ and weight $73.5 \pm$ $11.3 \mathrm{Kg}$ ) without any visible gait problem and able to walk unassisted without using a walking aid participated on the test. These measures of height and weight were selfreported by individuals.

After excluding the resulting signals that were not good, only 9 individuals were considered (explanation provided on Chapter 4, Section 4.4). For these participants, height, leg length and foot length was measured experimentally (mean height $1.75 \pm$, mean leg length $84.5 \pm 5 \mathrm{~cm}$ and mean foot length $28 \pm 1 \mathrm{~cm}$ ), and a report of their shoe number on the European system was requested.

Their height was measured by requesting individuals to stand next to a wall and marking their height on a piece of paper fixed on the wall.

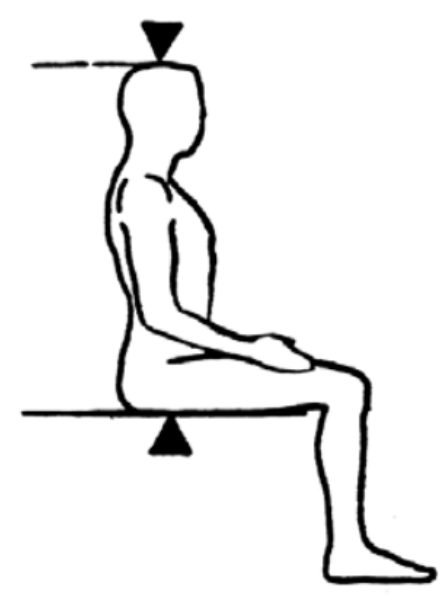

Figure 3.6: Sitting height. Source: [97].

Then, their leg length was also measured, using a process described by Bogin and Varela-Silva (2010) [97]. As it is difficult to measure the anatomical leg length in a human being, this measure was estimated from individual height and sitting height, which is the total structure that is comprised by head and trunk (Figure 3.6). The remaining portion of the body will be the length of the legs. Sitting height was determined by asking individuals to sit next to a wall, and marking their height on a piece of paper fixed on the wall. Leg length was determined as the difference between standing and sitting height. 
Foot length was measured with the shoes on. Persons were requested to place the foot perpendicular to a wall, and using a rule, the foot length was measured.

\section{Scenario}

The experimental setup comprised a walkway with 5 meters with distance markers placed on the ground (Figure 3.7).

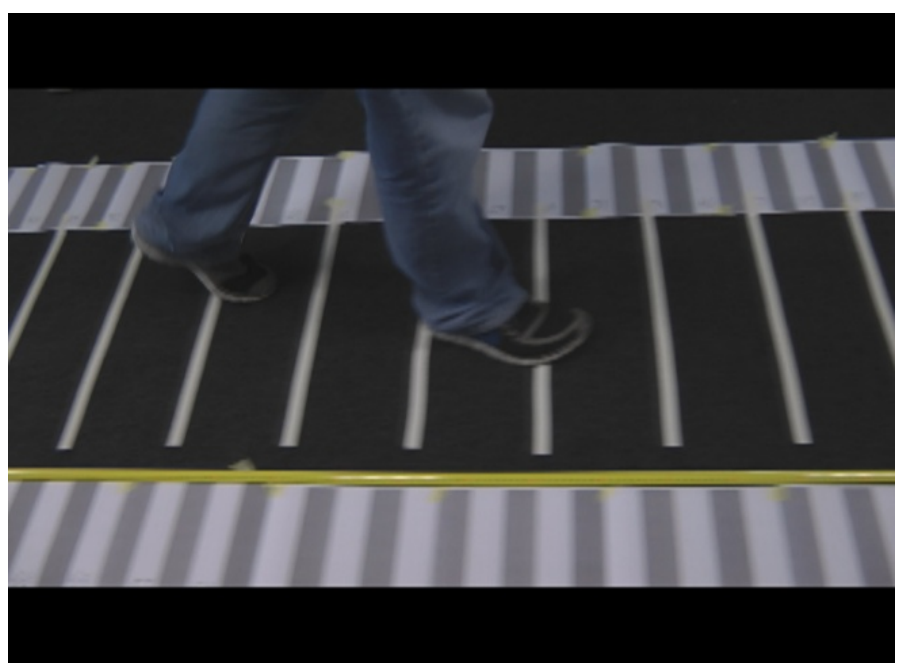

Figure 3.7: Tests scenario.

The phone was placed inside a case and adjusted around the pelvis using a belt. It was positioned with a known orientation relative to the ground and to the walking direction at the lower back of trunk of each participant (Figure 3.1), as described previously.

Subjects were asked to walk along the walkway until its end at three different selfselected speeds: comfortable normal pace, slower pace and a faster pace. Each test was repeated one time. The selection of three different self-selected speeds aimed to obtain a larger variability of signals, so that events detection and calculation of parameters could be done for different velocities.

Once again, the application from Fraunhofer AICOS was used. Subjects started walking immediately after the bip sound or after some seconds of quiet standing.

During each test, a simultaneous recording of a digital camera (recording 50 frames per second) parallel to the ground and of phone sensors was done. On Figure 3.7 a frame from the camera recording is provided.

\section{Data analysis}

Each video was analysed in order to obtain an estimative of the mean step length, duration and velocity. A frame by frame analysis was necessary in order to determine the time of 
each heel contact and each step length, using the information of the distance markers on the ground and time of each frame. IMTOOL MatLab ${ }^{\circledR}$ 's toolbox was used to estimate all steps length, as illustrated on Figure 3.8. Video information was used as a reference to evaluate the results obtained from sensors data.

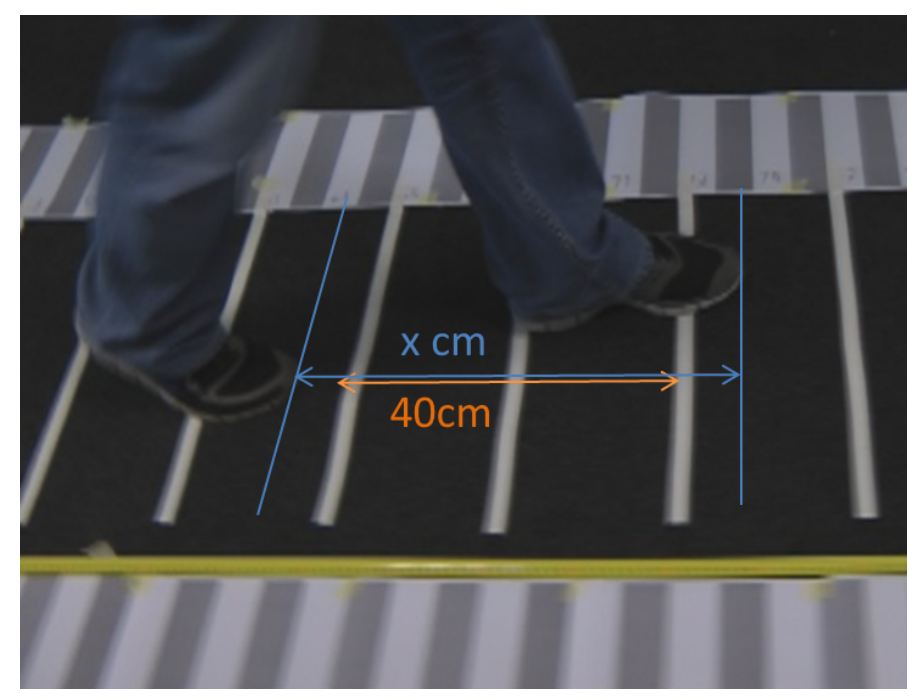

Figure 3.8: Illustration of step length estimation using video recording. A distance of $20 \mathrm{~cm}$ exists between two markers.

Mean step length was calculated as the mean length of each step during each trial. The mean step length was further confirmed using the total walking distance divided by the total number of steps. The mean step length calculated as the mean of each single step length was used as reference data.

For each step, also its duration (i.e. the time between two consecutive foot contacts) was determined using the video time information and the mean step duration was calculated. Mean step duration was also determined as the total duration of gait divided by the number of steps.

The velocity was calculated as the ratio between mean step length and mean step duration.

Only measures of the mean were used for comparison with results from sensors data, since some error is always present on the estimative provided by frame analysis. Also, as a reduced number of steps were available, the other measures were not calculated, since a low number of steps could not provide representative measures of variability.

\subsection{Risk Profile}

As purposed on Section 3.1, phone can also be used to build a risk profile that can change over time. According to Miller et al. (2002), single measures of physical performance 
or capacity for mobility do not enable the evaluation of person's confidence on balance, which is a potential barrier to mobility [46]. Also, results from Laessoe et al. (2007) support the idea that fall risk cannot be predicted solely assessing a physical performance, because falling has a multifactorial nature [60].

Later, the time-dependent risk factors can be used to estimate a likelihood of falling, cf. Section 2.7 of Chapter 2.

But first, the risk factors included in the multidimensional risk screening must be identified, so that a self-perceived risk profile can be built. An important requirement needs to be considered, which is the self assessment nature of the tests to be performed on the phone.

Based on the current medical validated tests and risk factors for falling, a set of tools were selected in order to construct a risk profile essentially based on self-reported risk factors, which include fall history, medication use, psychological function, confidence on mobility and in performing ADL, dizziness and fear of falling. This profile would give rise to an overall health status, which could be complemented by the performance measure of gait evaluation.

A summary of the literature review regarding the most suitable tests to be used to assess different self-reported risk factors is presented on the next table (Table 3.1).

Table 3.1: Self reported tools and risk factors.

\begin{tabular}{|l|l|l|}
\hline Tool & Risk(s) Factor(s) & Observations \\
\hline \hline $\begin{array}{l}\text { Activities-Specific Balance } \\
\text { Confidence Scale (ABCS) }\end{array}$ & Balance confidence & $\begin{array}{l}\text { Perceived capabilities on performance of } \\
\text { situation-specific activities, e.g. climbing stairs } \\
\text { or reaching above the head. }\end{array}$ \\
\hline Falls Efficacy Scale (FES) & Balance confidence & $\begin{array}{l}\text { Evaluates confidence in performing common ac- } \\
\text { tivities, e.g. take a shower or get dressed. }\end{array}$ \\
\hline $\begin{array}{l}\text { Modified Falls Efficacy Scale } \\
\text { (MFES) }\end{array}$ & Balance confidence & $\begin{array}{l}\text { Modified version of FES; includes additional } \\
\text { items related to the confidence in performing out- } \\
\text { door activities; greater internal consistency and } \\
\text { response variability than the original FES. }\end{array}$ \\
\hline $\begin{array}{l}\text { Functional Status Question- } \\
\text { naire (FSQ) }\end{array}$ & $\begin{array}{l}\text { Disability on phys- } \\
\text { ical, psychological, } \\
\text { social and role } \\
\text { functions }\end{array}$ & $\begin{array}{l}\text { It comprises six summary scale scores, assessing } \\
\text { basic ADL, intermediate ADL, mental health, } \\
\text { work performance, social activity and quality of } \\
\text { interaction; individuals scores can identify prob- } \\
\text { lematic areas. }\end{array}$ \\
\hline The Barthel Index (BI) & ADL autonomy & $\begin{array}{l}\text { Assess the patient's dependency when perform- } \\
\text { ing ADL. }\end{array}$ \\
\hline Katz ADL & ADL autonomy & $\begin{array}{l}\text { Assess person's ability to perform basic ADLs } \\
\text { independently. }\end{array}$ \\
\hline Lawton IADL & IADL autonomy & $\begin{array}{l}\text { Assess more complex ADLs, e.g. shopping, } \\
\text { cooking or self-medicating. }\end{array}$ \\
\hline
\end{tabular}

The Health Status Questionnaire (HSQ), described in Appendix A, will serve as a model for the construction of a risk profile. This scale assesses different risk factors (i.e. history of falling, limitations in mobility, ADL and IADL, medication use, diseases, 
dizziness, muscle weakness, pain, mental status, fear of falling and self-efficacy) and the categorization between high and low risk of falling is done based on the number of risks factors that are present.

Having in consideration the Table 3.1, the HSQ and the physical test described earlier (i.e. the gait test), a risk profile could be constructed based on all of the risk factors that each tool pretends to assess. Since there are some tools that are assessing the same kind of risk, a selection of tools is necessary, based on the prediction ability of each one.

History of falling can be assessed as suggested by HSQ, simply asking the person about previous falls, and identifying if recurrent falls were present. Further detail can be provided, based on the FROP-Com questionnaire. This can include asking about the occurrence of injury during a past fall in the past 12 months. Details can include the time, location, direction and cause of fall (as represented in Figure 3.9). FROP-Com is suggested in [28].

\begin{tabular}{|c|c|c|}
\hline \multicolumn{2}{|l|}{ History of falls (0-3points) } & SCORE \\
\hline $\begin{array}{l}\text { - Number of falls in the past } 12 \text { months? } \\
\qquad \ldots \ldots \ldots \ldots \ldots \ldots \ldots \ldots \ldots \ldots \ldots \ldots \ldots \ldots \ldots \ldots \ldots \ldots\end{array}$ & $\begin{array}{l}\square \text { Nil in } 12 \text { months }(0) \\
\square 1 \text { in the last } 12 \text { months (1) } \\
\square 2 \text { or more in } 12 \text { months (2) } \\
\square 1 \text { or more requiring hospitalisation (3) }\end{array}$ & \\
\hline $\begin{array}{l}\text { Was an injury sustained in any of the } \\
\text { fall/s in the past } 12 \text { months? } \\
\text { (rate most severe injury due to a fall in } \\
\text { the past } 12 \text { months) }\end{array}$ & $\begin{array}{l}\square \text { No (0) } \\
\square \text { Minor injury, did not require medical attention (1) } \\
\square \text { Minor injury, did require medical attention (2) } \\
\square \text { Severe injury (fracture, etc) (3) }\end{array}$ & \\
\hline \multicolumn{2}{|c|}{$\begin{array}{l}\text { - Describe the circumstances of the most recent fall in the past } 12 \text { months. } \\
\text { Time of fall: AM / PM (please circle) } \\
\text { Location of fall: inside home / outside home / community } \\
\text { Direction of fall: left / right / forward / backward / down / can't remember / other } \\
\text { Cause of fall: trip / slip / loss of balance / knees gave way / fainted / feeling dizzy or giddy / } \\
\text { Injuries: alcohol or meds / fell out of bed / unknown }\end{array}$} & \\
\hline
\end{tabular}

Figure 3.9: Part of FROP-Com assessing history of falling. Adapted from: [98].

Balance confidence, which is assessed by ABCS, FES and MFES, can also be part of the risk profile. On the three scales the person is asked to rate each activity according to his/her confidence level in its performance. MFES is recognized to provide better results than FES. For ABCS, cutoff values to discriminate high risk from low risk people are provided. As referred on Appendix A, a cut-off score of $67 \%$ resulted in $84 \%$ sensitivity and $87 \%$ specificity in correctly classifying fallers and non-fallers in a cross-sectional study of older people living in the community [99]. Then, the ABCS could be used to easily identify the presence of a low self-confidence in performing common activities.

Limitations in performing ADL and IADL can be assessed using different scales. Although FSQ include scales to evaluate these risk factors, the others were specifically designed to assess them. Then, BI and the Katz ADL can assess difficulties in ADL. Katz 
ADL seems to be more simple and fast, so it can easily be adapted to the phone and used by person. According to [100] and [19], a Katz ADL score of 2 or less indicates severe functional impairment, so that this cutoff can be used to identify the presence of risk factor.

Lawton IADL is used to assess the limitations in performing IADL (cf. Appendix A).

Medication use can be evaluated based on the HSQ. In this tool, polypharmacy, if present, is identified. Medications with higher correlation with risk of falling is also assessed, providing a more complete information about medications use.

FROP-Com also assess medications. It can therefore also be used as a model to assess medications in older people. This part of the scale is showed on Figure 3.10.

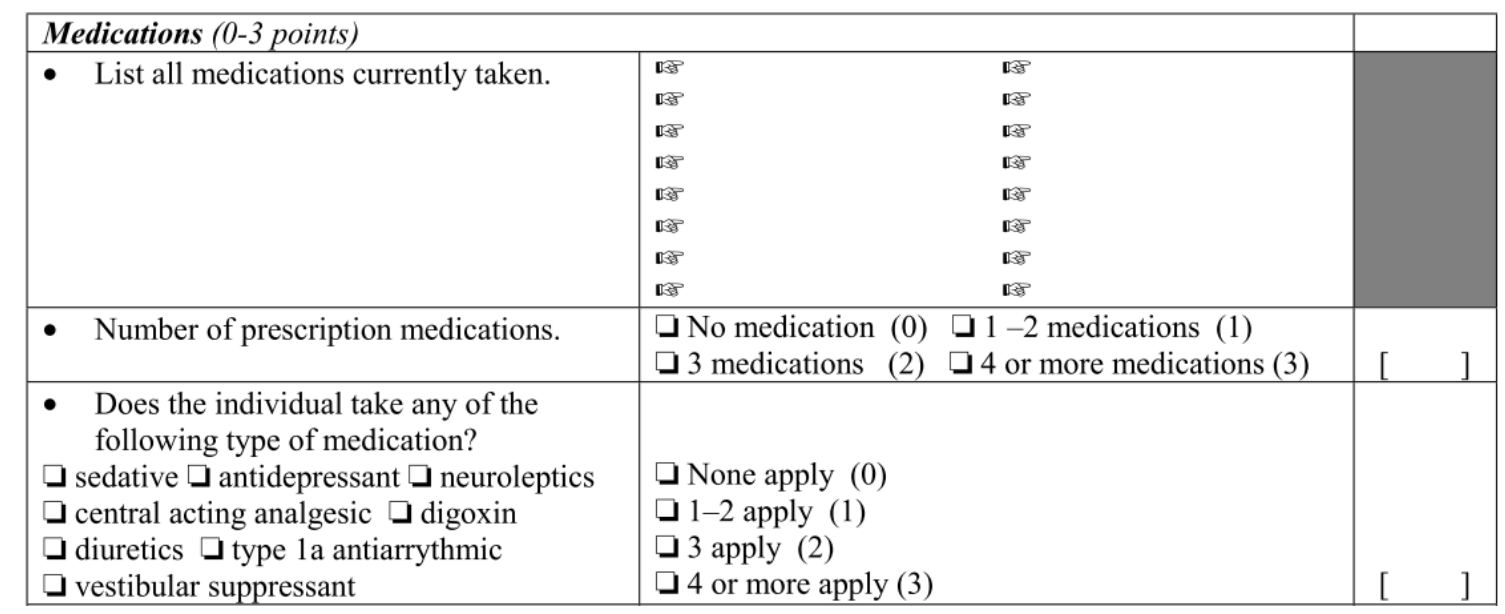

Figure 3.10: Part of FROP-Com assessing medications. Adapted from: [98].

Assessing the medications currently taken by older person would be useful to study medications in relation to the risk of falling over time. This would be important to identify which medication was potentially responsible for a risk augment. As suggested in FROPCom, a list of medications could be built.

Medical conditions could also be assessed. This would be based on the person knowledge or perception of his/her own diseases. The most relevant conditions for an higher risk of falling could be explicitly asked to the person, as happens on HSQ and FROP-Com.

On Figure 3.11, the assessment of medical conditions in FROP-Com is showed. At this scale, a list of medical conditions affecting balance or mobility is identified. A list of medical conditions is also present on HSQ.

FSQ can also provide ways to assess other important risk factors, e.g. psychological function, social activity and quality of interaction (see Appendix A). For all these three scales, warning zones are available, so that the presence of risks can be evaluated.

At least, the results of the gait test described earlier can also give important information of risks. Identification of gait or mobility problems can be identified through this test, 


\begin{tabular}{|l|l|l|}
\hline Medical conditions (0-3 points) & & \\
\hline $\begin{array}{l}\text { Does the individual have a chronic } \\
\text { medical condition/s affecting their }\end{array}$ & & \\
balance \& mobility? & $\square$ None apply (0) & \\
$\square$ Arthritis $\square$ Respiratory condition & $\square 1-2$ apply (1) \\
$\square$ Parkinson's Disease $\square$ Diabetes & $\square 3-4$ apply (2) & \\
$\square$ Dementia $\square$ Peripheral neuropathy & $\square 5$ or more apply (3) & \\
$\square$ Cardiac condition $\quad \square$ Stroke & \\
$\square$ Other neurological conditions & \\
$\square$ Lower Limb Amputation. $\square$ Osteoporosis & Osteoporosis: $\square$ Unknown $\square$ does not have & \\
$\square$ Vestibular Disorder $\square$ Other dizziness & \\
$\square$ Back pain $\square$ lower limb joint replacement & & \\
\hline
\end{tabular}

Figure 3.11: Part of FROP-Com assessing medical conditions. Adapted from: [98].

so that this risk factor can be included on the risk profile. In particular, gait velocity lower than $70 \mathrm{~cm} / \mathrm{s}$ was identified as a predictor of increased fall risk, according with [56] and also a poor/fair mobility indicator, according with [57].

Risk profile would then lead to the following result (Table 3.2):

Table 3.2: Risk profile.

\begin{tabular}{|c|c|}
\hline Risk Factor & Risk profile \\
\hline Fall History (Have you fallen during the past 12 months?) & $\overline{\mathrm{Y} / \mathrm{N}}$ \\
\hline ADL difficulties (Katz ADL score $\leq 2 ?$ ) & $\mathrm{Y} / \mathrm{N}$ \\
\hline IADL difficulties (IADL score $<8 ?)$ & $\mathrm{Y} / \mathrm{N}$ \\
\hline Gait/Mobility difficulties (Velocity $<70 \mathrm{~cm} / \mathrm{s} ?$ ) & $\mathrm{Y} / \mathrm{N}$ \\
\hline Balance confidence (ABCS score < 67\%?) & $\mathrm{Y} / \mathrm{N}$ \\
\hline Medication Use & \\
\hline Polypharmacy (Do you take 4 or more medications?) & $\mathrm{Y} / \mathrm{N}$ \\
\hline Cardiovascular system medications (diuretics, anithypertensives) & $\mathrm{Y} / \mathrm{N}$ \\
\hline Psychoactive medications (sedatives, antidepressants) & $\mathrm{Y} / \mathrm{N}$ \\
\hline Musculoskeletal system medications (narcotics, corticosteroids) & $\mathrm{Y} / \mathrm{N}$ \\
\hline Other (hypoglycaemics, allergy, cold medications) & $\mathrm{Y} / \mathrm{N}$ \\
\hline Medical Conditions & \\
\hline Musculoskeletal (arthritis, ... ) & $\mathrm{Y} / \mathrm{N}$ \\
\hline Neurological (stroke, Parkinson’s, ... ) & $\mathrm{Y} / \mathrm{N}$ \\
\hline Heart diseases (postural hypotension, arrhythmias, unstable, ...) & $\mathrm{Y} / \mathrm{N}$ \\
\hline Diabetes & $\mathrm{Y} / \mathrm{N}$ \\
\hline Dizziness & $\mathrm{Y} / \mathrm{N}$ \\
\hline Psychological function (FSQ score $\leq 70$ ?) & $\mathrm{Y} / \mathrm{N}$ \\
\hline Social activities (FSQ score $\leq 78 ?)$ & $\mathrm{Y} / \mathrm{N}$ \\
\hline Quality of interactions (FSQ score $\leq 69$ ?) & $\mathrm{Y} / \mathrm{N}$ \\
\hline
\end{tabular}

This risk profile will vary with time. Each assessment of risks and time of assessment has then to be recorded. Later, a likelihood of falling could be calculated, based on the identified risks.

Monitoring of fall risk and risk profile over time could then be possible. 


\section{Chapter 4}

\section{Results and Discussion}

On this section, some results concerning the algorithm development, events detection and parameters estimation are presented. These results provide the necessary data to evaluate the algorithm performance on events detection and parameters estimation, and also to discuss the possibilities to adapt this system to the phone, for fall risk prediction purposes. This also enables the identification of gaps that need improvement in order to make a functional and clinically useful application.

A simultaneous discussion of results is also done in this chapter.

\subsection{Inertial sensors signals}

Phone's inertial sensors signals obtained during gait could be successfully extracted to the computer for further analysis.

Figure 4.1 show typical acceleration and linear acceleration signals obtained during gait, on the three directions: forward, lateral and vertical. Signals don't have a fixed sampling rate, as the phone only registers a value when it changes.

As can be observed, linear acceleration signals are adjusted around zero and present a similar pattern when comparing with acceleration. Signals present repetitive patterns, as is expected, since gait is a repetitive activity. In general, all the acceleration signals extracted during gait have a pattern that is similar to the one encountered on the literature, satisfying the theory.

Forward linear acceleration signal was used on the first part of the process to detect zero crossings, as already explained, because linear acceleration present more emphasised zero crossings and is already adjusted around zero (see Figures 4.1a and 4.1b). 


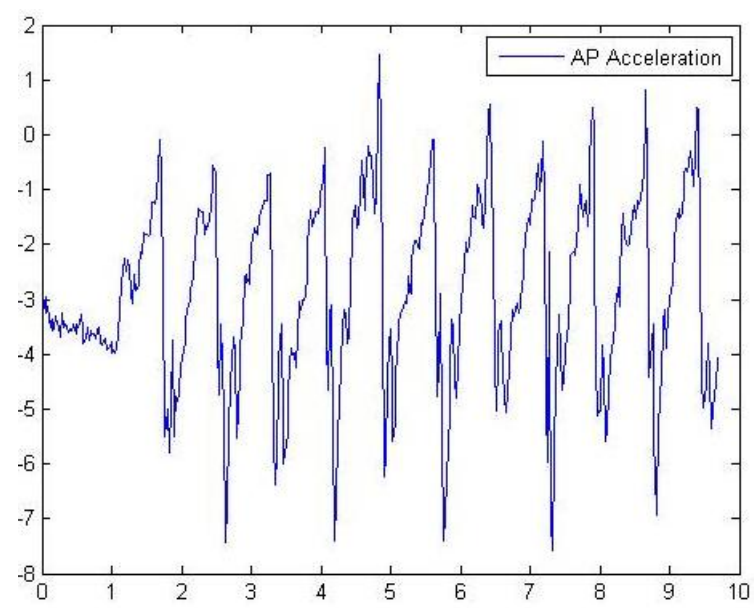

(a) Forward Acceleration

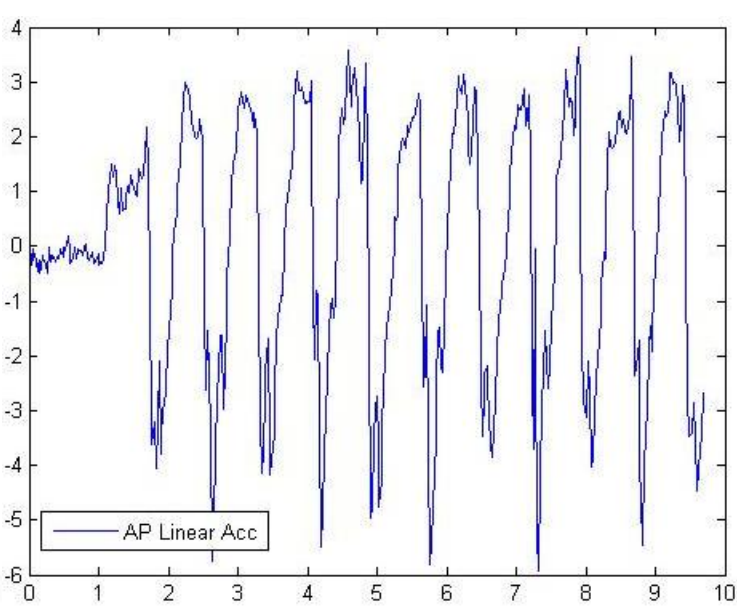

(b) Forward Linear Acceleration

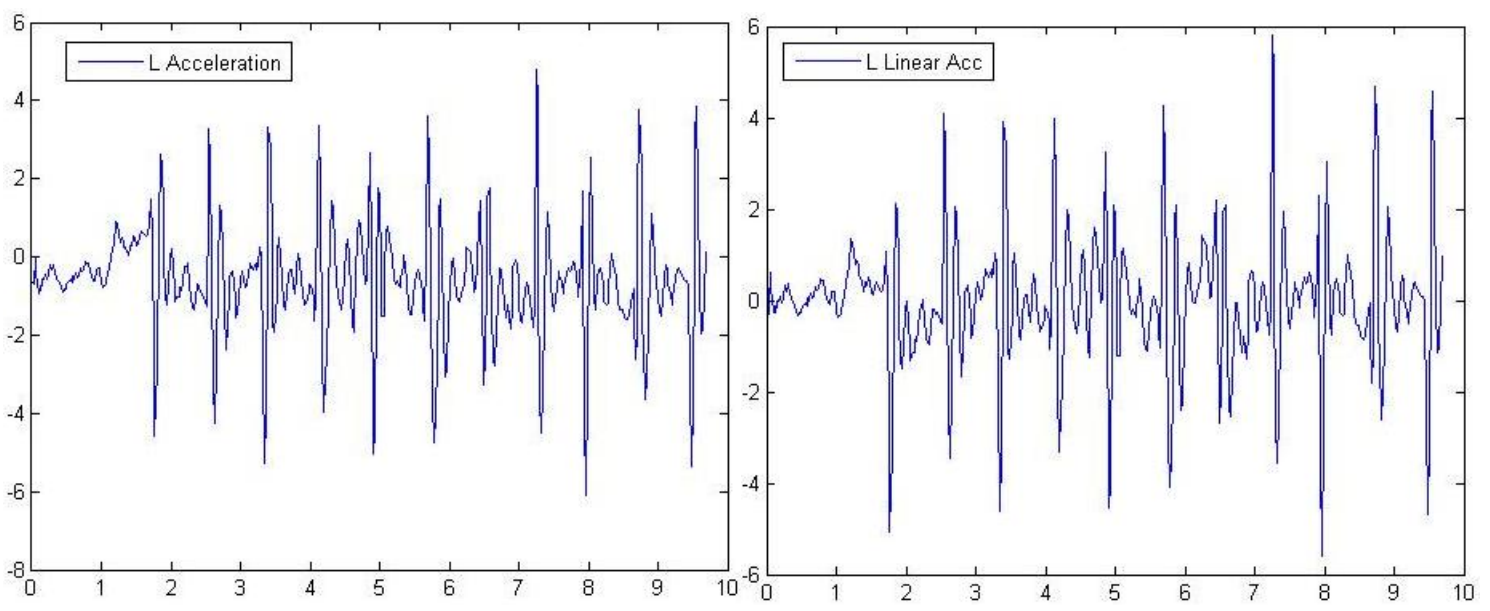

(c) Lateral Acceleration

(d) Lateral Linear Acceleration

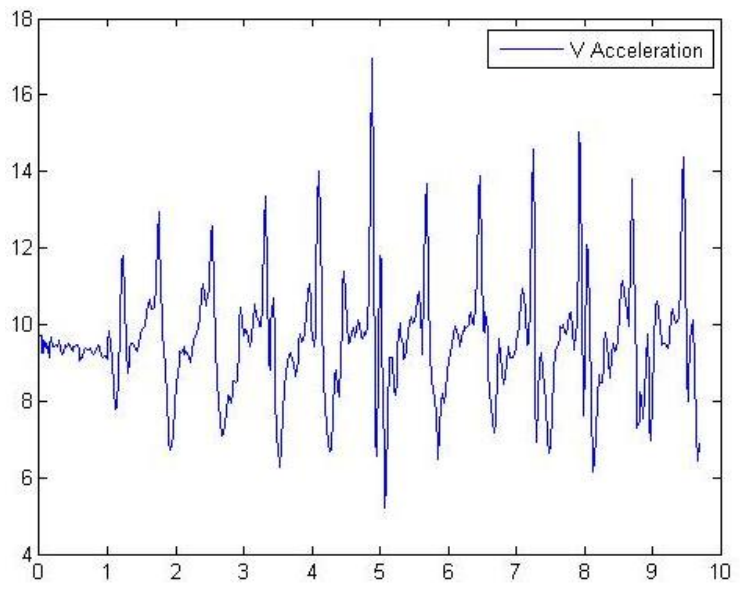

(e) Vertical Acceleration

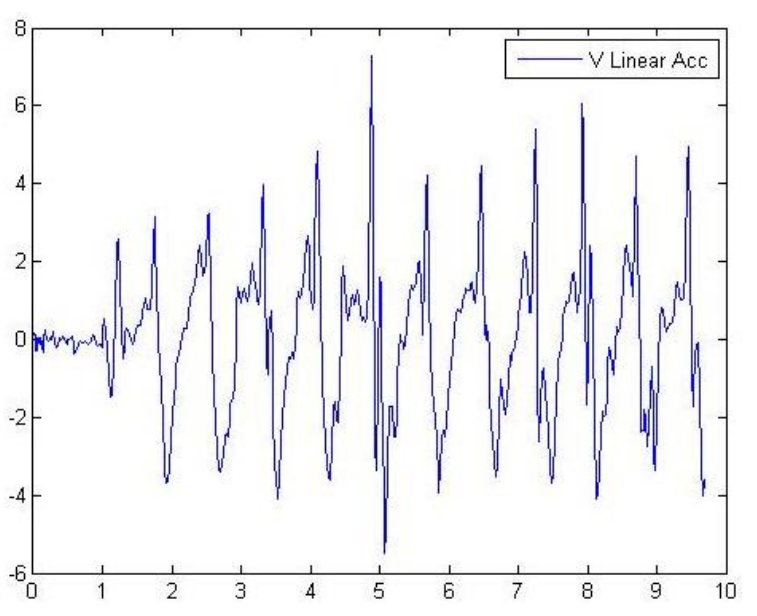

(f) Vertical Linear Acceleration

Figure 4.1: Inertial sensors signals obtained from phone sensors. 
Foot contacts events, described as peaks before zero crossings on acceleration, are not as marked in linear acceleration as they are on acceleration signal (Figures 4.1a and 4.1b). Acceleration was then used to detect these events, following the literature descriptions.

Lateral accelerations have some highly irregular parts of signal (as described in [72] and [71]), which also happens on lateral linear acceleration (Figures 4.1c and 4.1d). As linear acceleration is already adjusted around zero and its peaks are more pronounced, it was used to discriminate left from right foot contacts.

Vertical acceleration do not presented a pattern as simple as reported on the literature (see Figure 2.21, on Section 2.9). Vertical linear acceleration, however, had their peaks more pronounced, and, for that reason, it was used to detect toe offs, as described earlier. Also, the vertical linear acceleration had already excluded the static component of acceleration, which is the gravity constant.

However, recorded signals not always presented the desired characteristics. Some of them presented quite sporadic characteristics, being difficult to visually identify the normal gait events. An example of this kind of signal is presented on Figure 4.2.

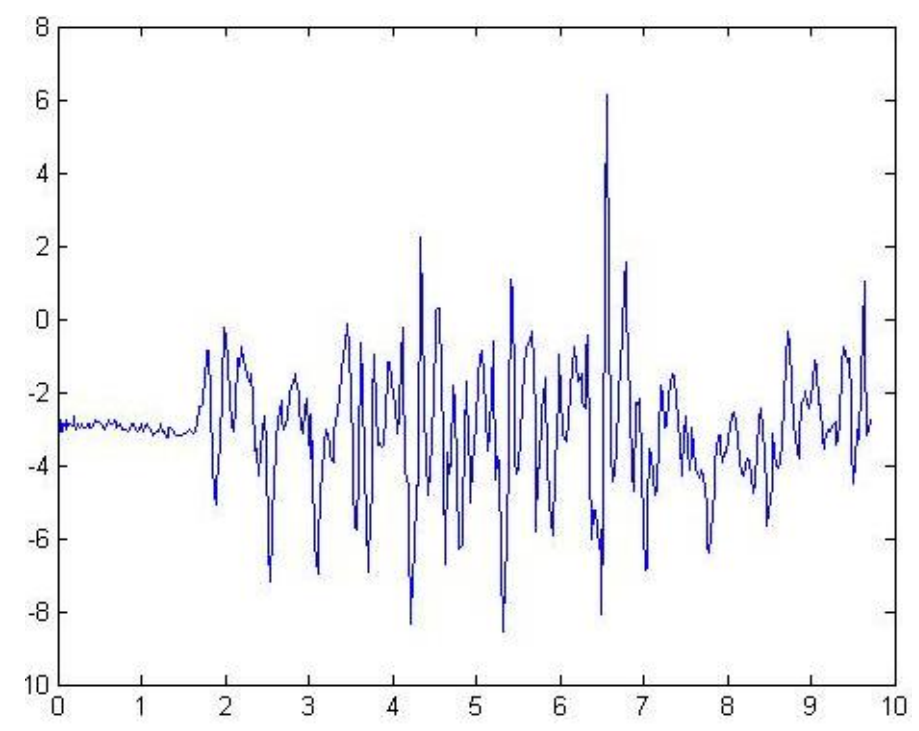

Figure 4.2: Example of a bad forward acceleration signal.

This did not happened always on the same person, so it may not identify a different gait pattern. Instead, the signal can have these kind of characteristics because of a bad positioning of phone on the lower back of trunk, i.e. its orientation could not be coincident with the reference axis from phone sensors. This would imply that other components of acceleration are measured along the wrong axis, so that different signal characteristics are obtained at the end. This phenomenon is illustrated on Figure 4.3, where a rotation of the phone is exemplified.

Also, the belt could not be very well adjusted around pelvis, so that phone could not 


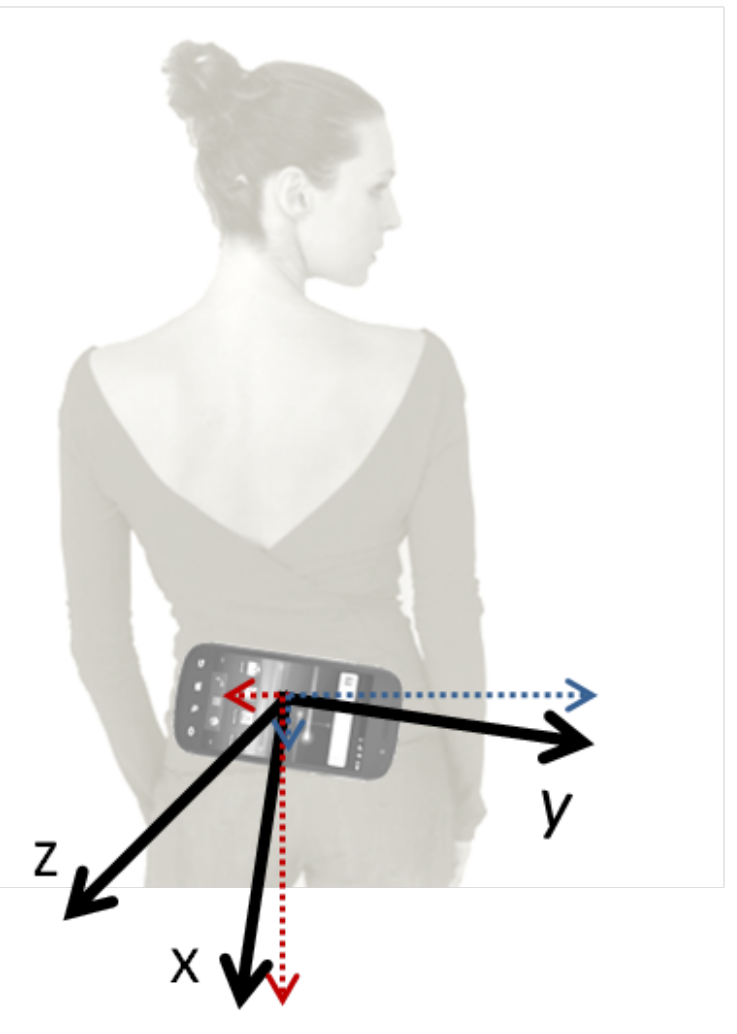

Figure 4.3: Illustration of acceleration errors due to wrong phone's orientation. In this case, theoretical vertical direction $(x)$ would also be measuring lateral components and lateral direction (y) would be quantifying vertical accelerations.

be stable, introducing measured accelerations corresponding to vibrations that are not characteristics of gait.

During gait, an particularly if phone is not properly placed, some rotations can occur on the phone, as person can be, for example, tilted on the forward direction, or a varying tilt can be observed as a consequence of gait. This introduces errors on the measurement, as the phone rotation leads to the recording of acceleration components that are not expected to be recorded.

\subsection{Events detection}

The events detection method presented earlier was used to detect events on gait signals. At this section, the results obtained in each step are presented. To illustrate the results of each algorithm step, the same test (i.e. quiet standing followed by walking) is used.

Figure 4.4 shows the zero crossings that were detected on linear acceleration signal.

As can be perceived, there are lots of zero crossings that are detected that are not representative of zero crossings after foot contacts, e.g. zero crossings at the beginning of 


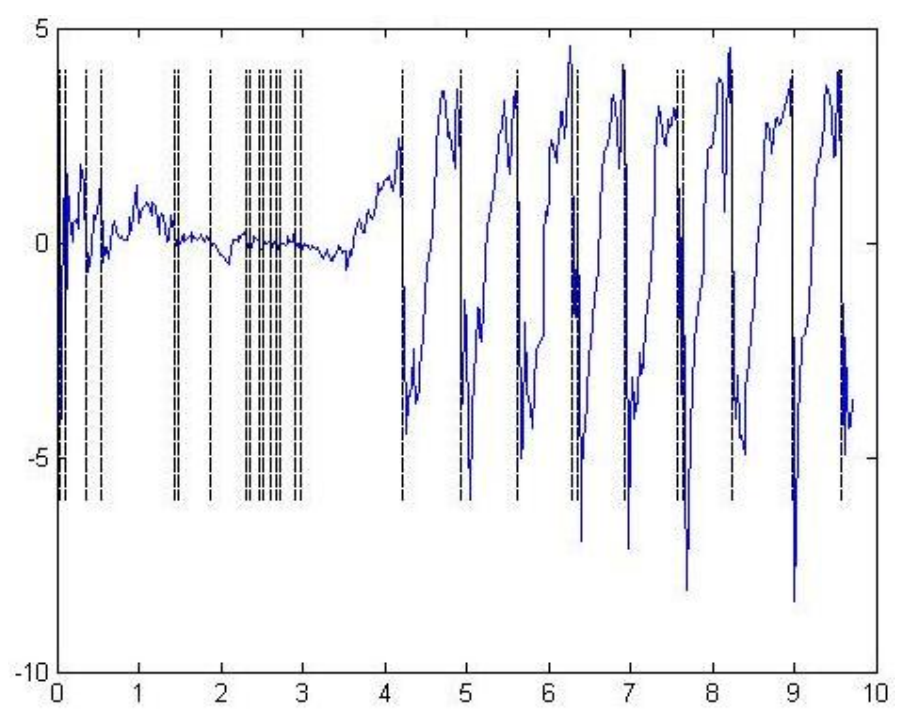

Figure 4.4: Zero Crossings detection in a real forward linear acceleration signal.

the test, and zero crossings due to noise (Figure 4.4). From this signal it can be perceived the need to eliminate the zero crossings without interest.

So, first, zero crossings which could be due to noise were eliminated, maintaining only the zero crossings with a preceding maximum above a given threshold (fixed at $0.5 \mathrm{~m} / \mathrm{s}^{2}$ ). This threshold was set very low, so that maximums representing foot contacts were not eliminated by confusing with noise. After this process, the maintained acceleration maximums were the ones represented on Figure 4.5.

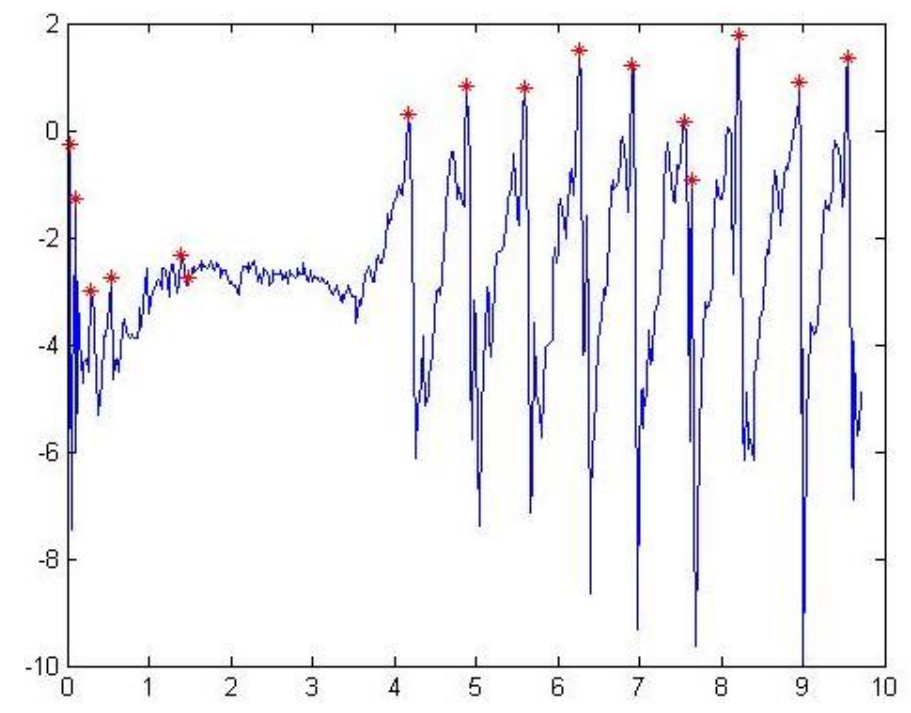

Figure 4.5: Maximums maintained after eliminating those due to noise.

As can be observed on Figure 4.5, still some maximums not representing foot contacts 
were present, e.g. those happening at the first part of signal. Then, a process using standard deviation was used (as described on Section 3.3). On the next Figure 4.6, the sliding SD of acceleration signal is shown.

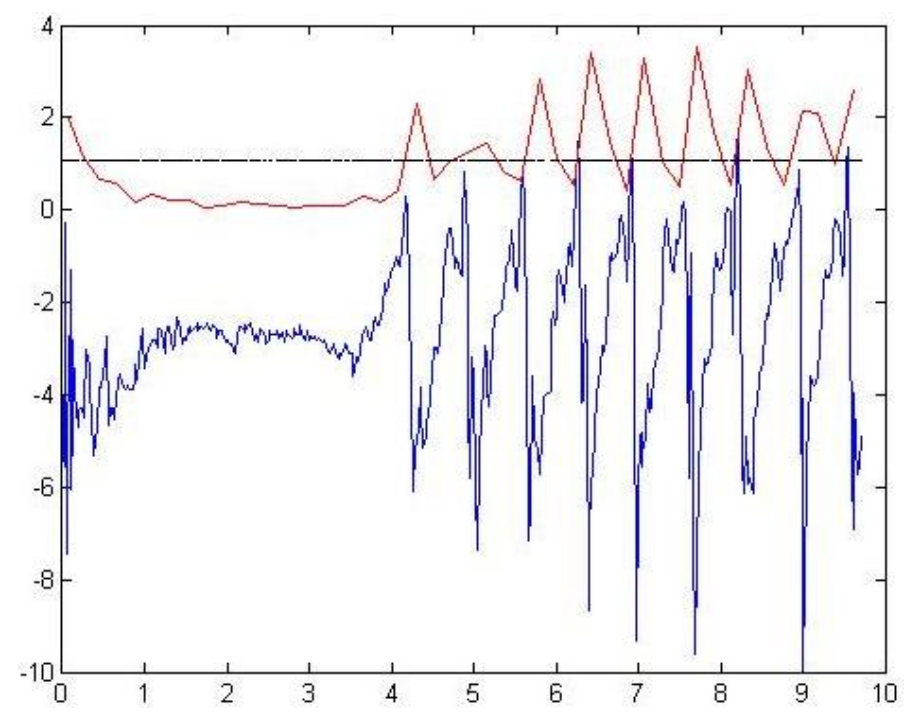

Figure 4.6: Acceleration (blue) and Standard deviation (red) signals. The mean standard deviation is represented in black.

As can be observed, foot contacts only began after standard deviation started to increase, reaching a value that is higher than the standard deviation mean. Then, only the maximums obeying to this condition were conserved (Figure 4.7).

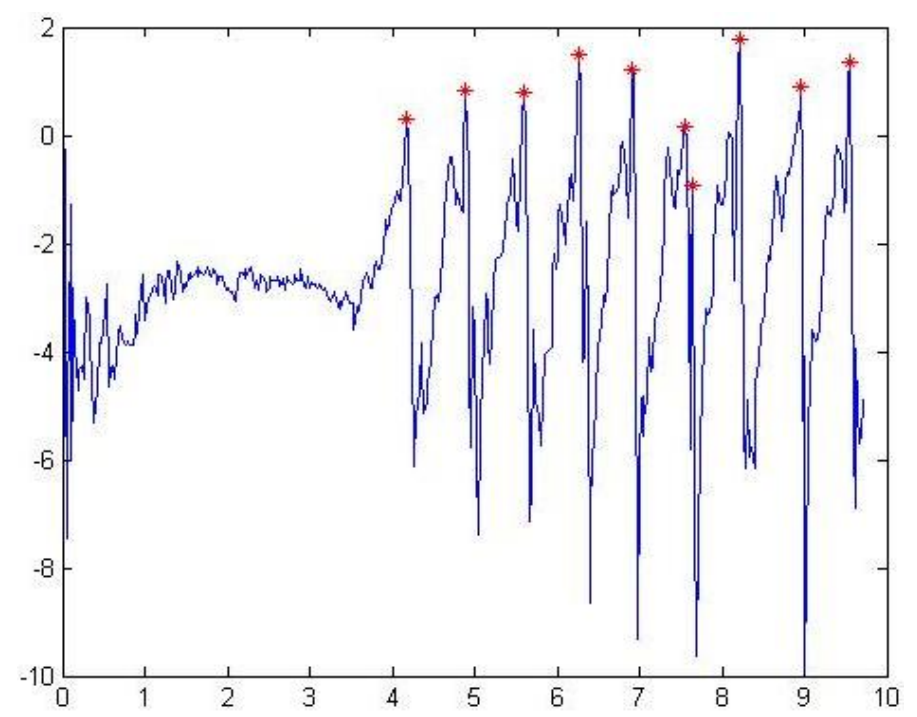

Figure 4.7: Retained maximums after eliminating those on the standing phase.

At this signal (Figure 4.7) there was still a maximum not representing a foot contact. 
Too close detections were eliminated using the method described at Section 3.3, considering that gait is a quite regular event. As a result, the foot contacts represented on Figure 4.8 were detected on the acceleration signal.

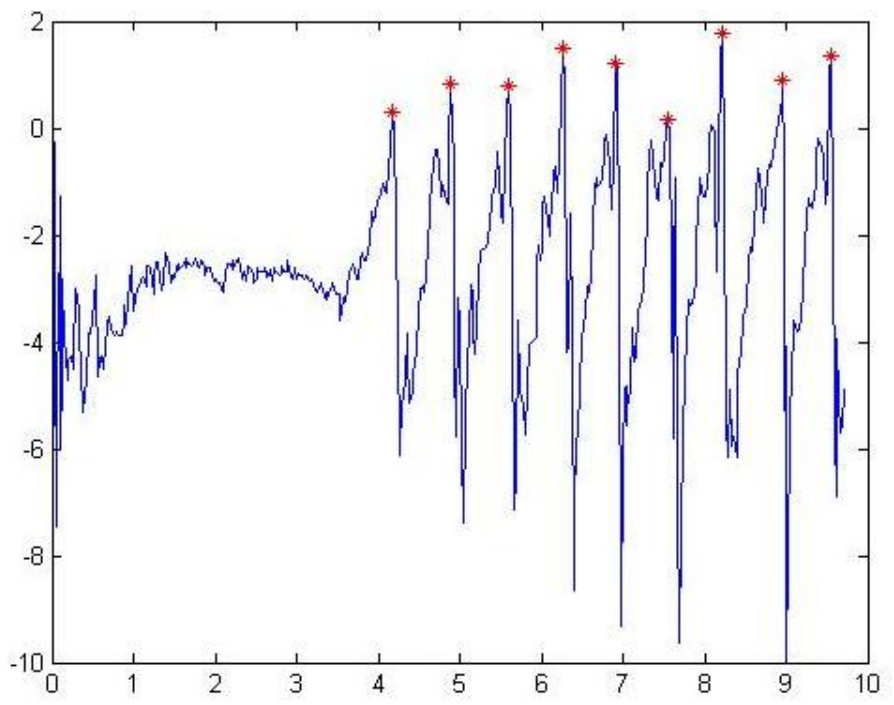

Figure 4.8: Foot contacts detection. blue: forward acceleration signal; red: foot contacts.

Perfect signals lead always to good detections of foot contacts. However, when signals presented different characteristics foot contacts detection was not correctly performed. Two examples are provided on Figure 4.9.

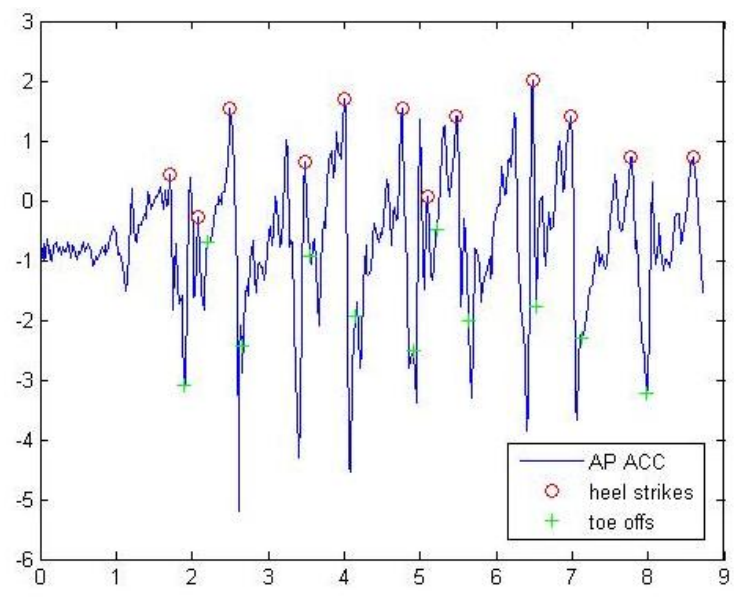

(a) Signal 1

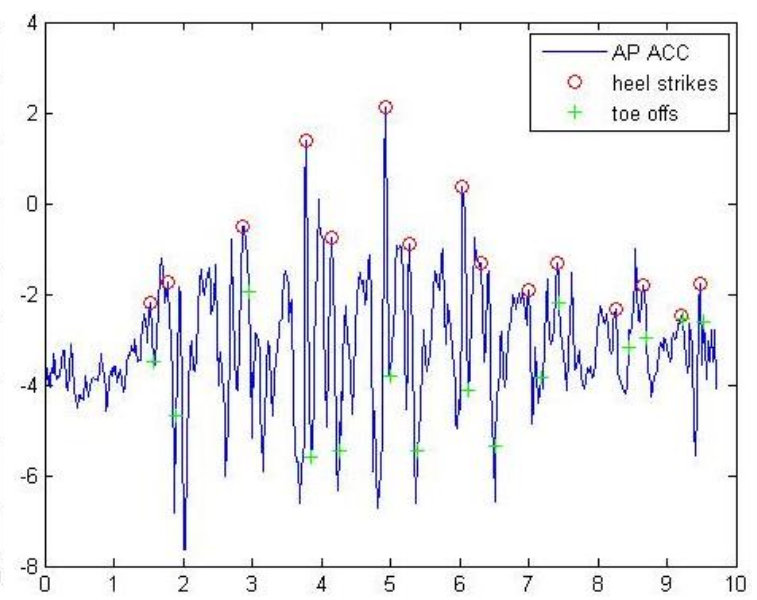

(b) Signal 2

Figure 4.9: Examples of bad foot contacts detection.

On Figure 4.9a, a regular signal pattern can be identified. However, some additional and pronounced peaks are present, and so some wrong detections are done. Figure $4.9 \mathrm{~b}$ illustrates another kind of failure. At this case, the acceleration signal is difficult to analyse, 
even visually. Then, algorithm also fails the foot contacts detection.

This poses some questions, regarding the kind of signals that will be encountered when older people walk. As more variability of gait is expected, according with the literature, it is also expected that signals with more variability are obtained, which poses some problems on the correct detection of foot contacts.

So, an improvement of foot contacts detection is required. In order to take the first steps on algorithm improvement, gravity signal was explored. As the gravity signal is a low pass version of acceleration and it is very smooth, it would be easier to analyse.

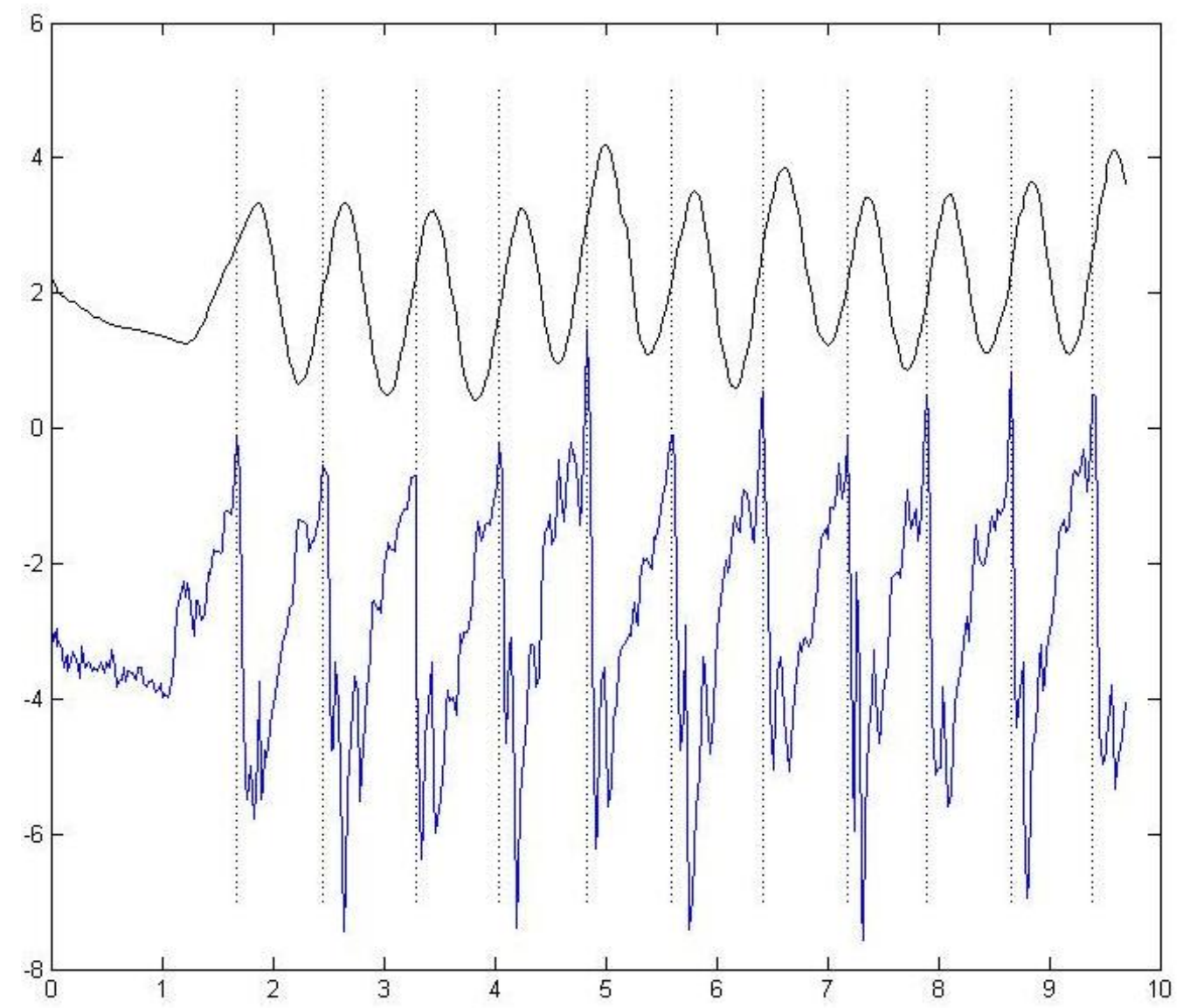

Figure 4.10: Forward acceleration (blue) and gravity (black). Vertical lines are the instants of foot contacts, detected by algorithm.

On the Figure 4.10, acceleration and gravity signals are plotted simultaneously. Vertical lines show the instants of foot contacts, as perceived by acceleration signal. As can be observed, the gravity peaks always happen after a foot contact, with a approximately constant delay (near $0.2 s$ ). As gravity is a filtered version of acceleration, this delay should be constant, as a result of the filtering process performed by the phone. Gravity could then be used to more efficiently and easily detect foot contacts, using the sensor fusion technology included in modern mobile phones. Further evaluation of this possibility would 
then be required.

Toe offs were detected on the vertical linear acceleration signal, using the definition provided on the literature. The same test from Figures 4.4 to 4.8 was used to illustrate toe offs detection on vertical linear acceleration signal (Figure 4.11).

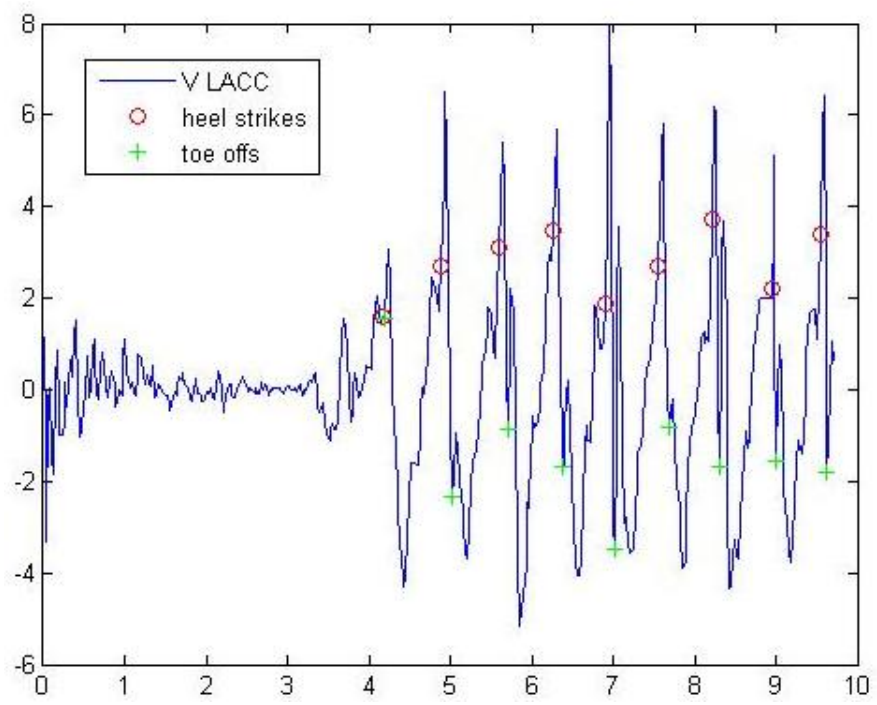

Figure 4.11: Toe offs and heels strikes on vertical linear acceleration.

As observed on Figure 4.8, the foot contacts were correctly detected. However, at this signal (Figure 4.11) foot contacts are not corresponding to a valley, as described on literature (Section 2.9). Minimum values after foot contacts were properly detected, however, it was impossible to evaluate if they effectively corresponded to toe offs.

\subsection{Integration}

As described on the methods, integration steps were necessary to determine displacement from acceleration. According with literature, this process requires signal filtering in order to eliminate signal drift (see Section 2.9, Figure 2.26).

Figure 4.12 shows the result of double integration of vertical acceleration using the corrections to correct drift (Figure 4.12b) and do not using them (Figure 4.12a).

It is clearly observed that do not applying filtration techniques leads to bad integrations of signal, since vertical position during gait is not always moving away as this graphic (Figure 4.12a) seems to inform. When applying filtering techniques, a vertical position varying periodically is obtained (Figure $4.12 \mathrm{~b}$ ).

On Figure 4.12b, a simultaneous plot of heels strikes and toe offs is showed. As expected, a circular path is obtained between these events, as predicted on the inverted pendulum model. 


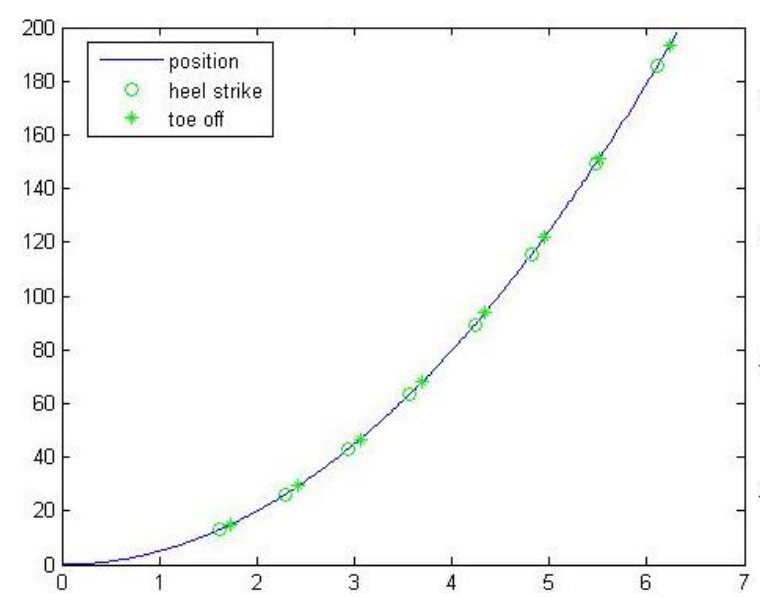

(a) Position (with drift)

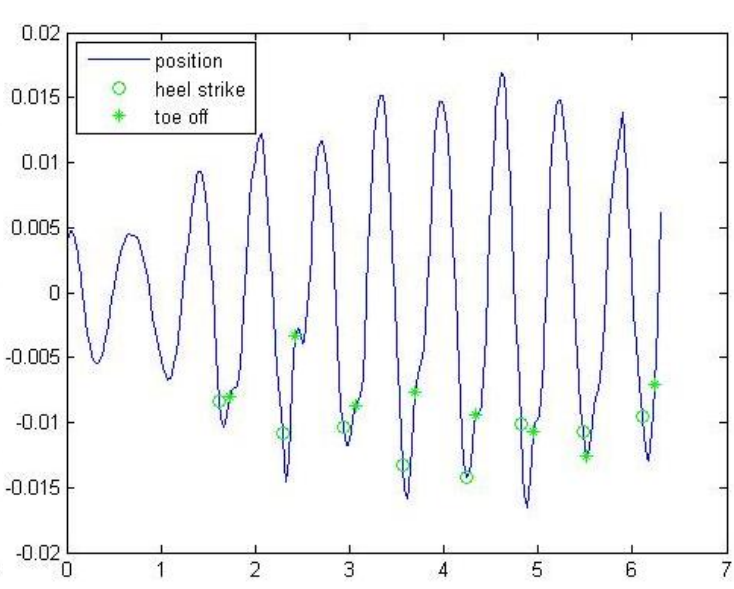

(b) Position (no drift)

Figure 4.12: Double integration of vertical acceleration.

\subsection{Step length, duration and velocity evaluation}

At this section, the results from the evaluation method (described on Section 3.4) are provided.

As already referred, some sensor data from some tests was not considered to become part of the study, because signals had a random like pattern, being difficult to identify the peaks corresponding to heel contacts during gait. Since the purpose of this evaluation is to validate step length and velocity estimation, this kind of signals was not considered, since bad detections of events were expected. Also, only the normal and slower speeds were used since the faster speeds were difficult to analyse using the videos from camera, because a low resolution in each frame was observed. Then, from the initial 84 tests, the 28 tests corresponding to the faster speeds were eliminated, and from these tests only 27 were considered for results evaluation. Then, only 9 individuals' data was analysed.

Using the measured parameters of leg length and foot length, the algorithm developed in MatLab ${ }^{\circledR}$ was used to estimate the mean step length of each trial. The maximum error from expected (i.e. from video results) and estimated (i.e. from sensors signal analysis) mean step length was $17 \%$ and the mean error was $7 \pm 5 \%$. Figure 4.13 shows the estimated values in relation to the expected ones.

Deviations of the measured mean step length compared with the expected values are either positive either negative and a tendency to over or underestimation is not observed.

This error seems to be acceptable, considering all the sources of error that can be present. First, the pendulum rule used to estimate mean step length is a rigid formula that was determined by approximation and simplification of gait signal. On the practice, more complex patterns of displacement, i.e. with more variability, are observed. For example, the leg is not maintained as a rigid pendulum, which could be translated in a variation 


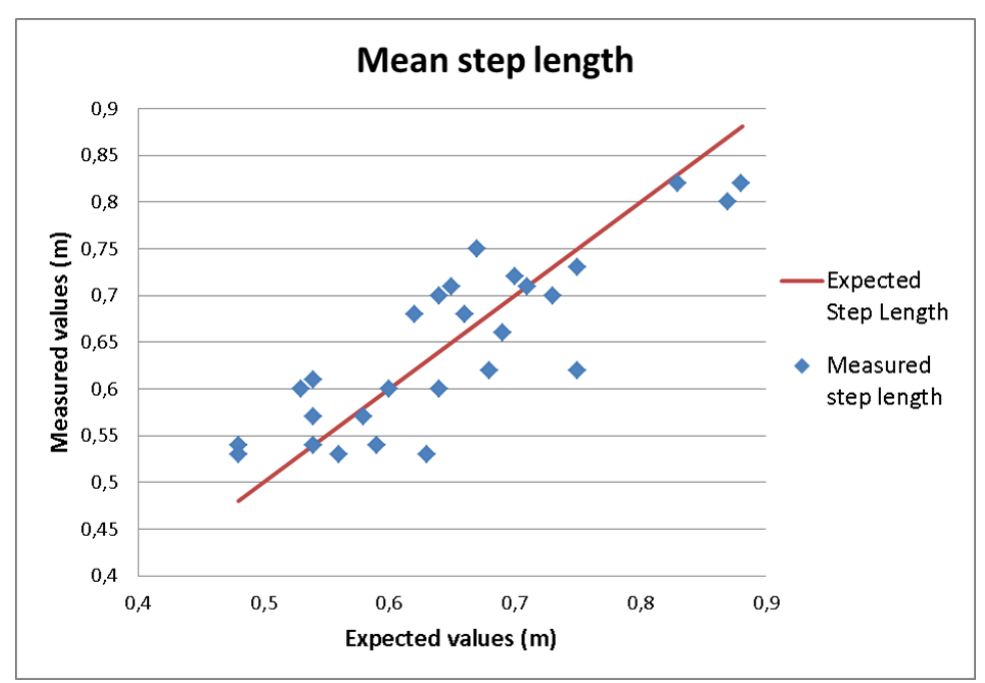

Figure 4.13: Expected and Measured mean step length.

of pendulum length. Also, displacement during double support was calculated by foot length, which not always was perfectly aligned with the direction of progression (this can be observed on Figure 3.7 of Section 3.4, where the right foot is slightly tilted). Second, errors on acceleration and position signals are present, so that others are introduced on step length estimation.

The maximum error from expected and estimated mean step duration was $3 \%$. No difference was observed in $41 \%$ of the considered tests. The mean error between measured and real values was $1 \pm 1 \%$. Expected and predicted mean step durations are represented on Figure 4.14.

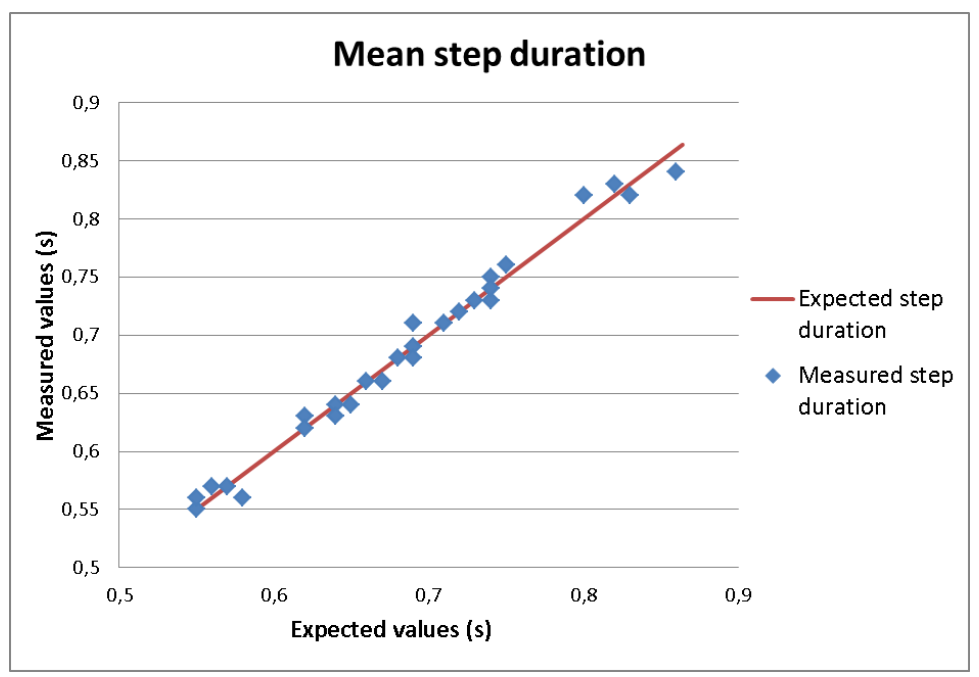

Figure 4.14: Expected and Measured mean step duration. 
Differences between measured and real values were very low, so that mean step duration was properly estimated using sensors data. This serves as an indication that peaks corresponding to foot contacts were properly detected.

The velocity, calculated as the mean step length divided by mean step duration is very dependent of the mean step length estimated values. The maximum velocity error is $15 \%$, and the mean error is $7 \pm 5 \%$.

Figure 4.15 shows the differences between expected and predicted velocity. The errors present on velocity are a reflection of errors on mean step length and duration. Although they are present, they are not very high, so that using phone's sensors to estimate gait velocity seems to be a reliable option when the main purpose is to discriminate high and low risk persons based on velocity. Velocity cutoffs reported on the literature do not seem very rigid and there are different authors reporting different cutoffs. A low error on velocity may then be not critical.

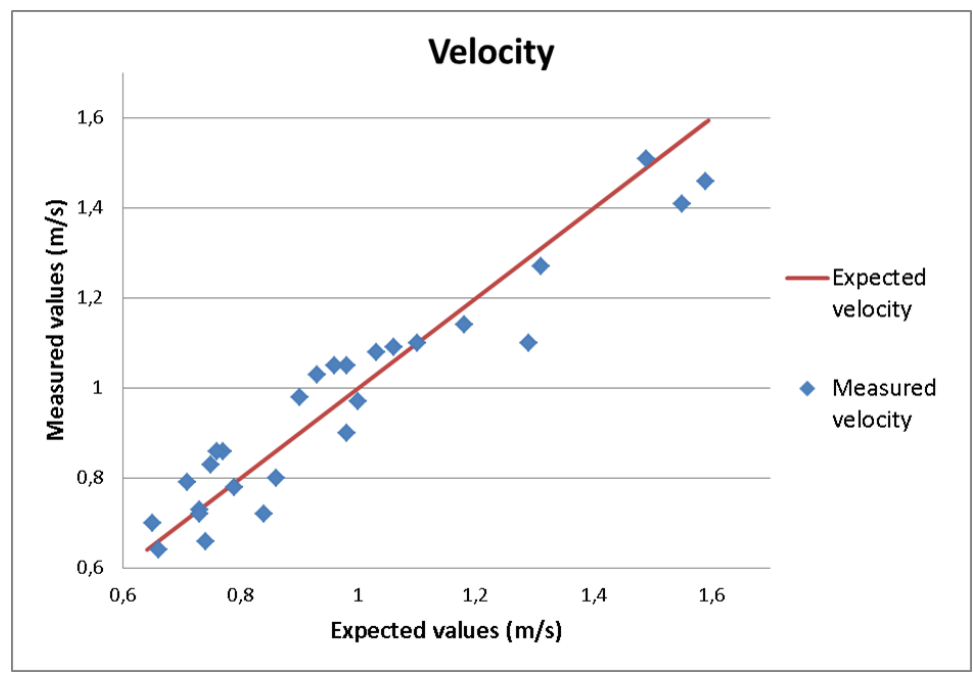

Figure 4.15: Expected and Measured velocity.

As the future objective of this project was assessing the risk of falling in an unsupervised manner, the length of the shoes (used to calculate the steps length) was calculated from the shoe number (which can be easily reported by older person). Some international shoe size conversion charts are available online (e.g. cf. [101]), and were used to estimate the length of the shoes of individuals. The maximum difference between real foot length and the foot length estimated from the shoe number in the European system was $4 \mathrm{~cm}$, corresponding to an error of $12 \%$.

Also, leg length was estimated from the reported height (which can be easily reported by older person). From the individuals data, the ratio between leg length and reported height has a mean value of $48 \pm 1 \mathrm{~cm}$. This ratio was used to estimate leg length from the reported height. Using these two estimated parameters (i.e. estimated leg length and 
estimated foot length), new estimated mean steps length and velocity were calculated, using sensors data. The mean error between expected and predicted mean step length (using estimated parameters) was $7 \pm 5 \%$. This error is on the same range as the error occurring when real leg length and foot length are used to calculate the predicted mean step length. However, further study is needed to better estimate the relation between height and leg length, since at this study a reduced number of persons were used. No relation between height and leg length was found on the literature.

The video recording method is not the best approach to validate steps length. In fact, some error is always present on the measurement, since low resolution of camera, bad positioning of auxiliary lines to determine step length or error on distance markers on the ground can be present. Making footprints on the ground would be a better approach for a reliable measurement of each step length. Due to time constraints, and in order to also estimate steps duration, video recording method was adopted, having in mind that some error may be present.

The other parameters (i.e. those related with variability) could not be validated using this method. In fact, the walkway used had a short size, so that few steps were available (between approximately 6 and 12 steps) and no enough time was available so that gait pattern could be stabilized from the beginning until the end of the walkway. Representative measures of variability couldn't be provided and a bigger walkway would be preferred.

\subsection{General discussion of results}

Detection of parameters from sensors signals could not be properly evaluated. To effectively validate foot contacts and toe offs, the use of traditional methods of gait analysis, such as cameras and force-plates would be required. Using these methods, the exact time of foot contacts and toe offs, and reliable measures of steps length could be determined. Unfortunately, this was not possible to perform in good time, so that only analysis based on the literature descriptions could be done.

The evaluation method performed was the most simpler that was encountered to give an estimative of the performance of sensor based gait analysis. From the results described, evidences exist that inertial sensors signal from the phone can be used to analyse gait. However, further exploration is need.

There are some considerations to take into account, regarding the operability of a future system. For example, the position of the phone during gait (i.e. lower back trunk) is not much comfortable, and can limit the possibilities of self-assessment of risk. Then, exploration of others places of attachment should be required.

Also, this poses some restrictions on the ways test will be performed, since the recording may include not only the recording of gait, but also the recording of other movements, e.g. putting the phone on the back or take the phone after performing the test. This would 
require that further processing of signal is done, in order to properly delimit the signal areas corresponding to gait. First steps were also taken in this sense, by eliminating the areas of signal corresponding to a quiet stance on the beginning of the test. However, if quiet stance is not straightly performed until the beginning of the test, the developed method fails.

Discrimination between high risk and low risk persons could not be evaluated. As indication, it can be referred that when performing the tests at the preferred speed (i.e. as required for risk stratification), all the persons walked at a velocity higher than $70 \mathrm{~cm} / \mathrm{s}$. As participants are not older persons, this was an expected result.

Further study is also need to explore the potentialities of risk profile creation. Evolution of risks over time, the weight that can be attributed to each risk factor (which may also vary over time) and the calculation of a likelihood of falling would be important topics of research. For now, identification of self-report risks is possible, using the existing medically validated scales.

Therefore, evidence exist that self-reported risks and identification of mobility or gait problems can be used simultaneously to screen for fall risk in community, as will be required to properly deal with the problematic of falls in older people. 


\section{Chapter 5}

\section{Conclusions and future Work}

With the increasing number of older people in the community, the problems related to ageing are requiring more and more attention. The falls are a common and increasing health problem that causes physical, psychological and social problems for the individual and increasing costs for society. Fall prevention strategies are therefore becoming increasingly important.

In this project, a study was done regarding the use of mobile phones as fall screening tools, aiming to improve the current fall prevention strategies.

Based on the current research, phone's inertial sensors were used to quantify some parameters of gait that were related with an increased risk of falling. Also, other important risk factors which could be determined by self-report from existing scales were identified, so that they could be assessed using the phone.

The most challenging parts of the work were the detection of foot contacts and also the determination of step length from inertial sensors signals. Some techniques were used to this purpose, including the approximation of gait by an inverted pendulum model. The mean error from expected and estimated mean step length was $7 \pm 5 \%$. For mean step duration, the error was $1 \pm 1 \%$ and for velocity $7 \pm 5 \%$.

The results obtained from sensors signals analysis suggest that they can be used to predict the risk of falling, based on the parameters quantifying gait characteristics. For now, only a low and high risk categorization could be possible, based on walking velocity. Later, a likelihood of falling could be assessed, using the information for all risk factors.

The use of mobile phone for fall risk screening purposes may be therefore a major strategy to improve fall prevention. Frequent assessment of risks can be done, and an evolution of risks can be obtained over time, which gives new insights about fall prevention strategies. Earlier application of prevention strategies can be done and the doctor can 
also modify the ineffective ones. Therefore, further development of the project would be of great value, not only for older persons, but also for society.

\subsection{Future work}

Having in consideration the results obtained in this project, some future work was identified to potentially improve fall risk screening methods. The following list details some of future work tasks.

- Exploration of techniques to improve the signals obtained using inertial sensors would be required. During gait, phone can be slightly tilted, or its orientation can change with walking, so that the local coordinate system of the phone is not aligned with the global axis system and other components of signals are measured on the three principal directions of gait (i.e. vertical, forward and lateral, in the direction of progression). Recording should then by independent of phone's orientation, so that much troubles do not happen when wrongly positioning the phone.

- Phone's positioning should be more comfortable, so that the exploration of other places of attachment should be required.

- It would be important to validate foot contacts detection, step length estimation and also the other parameters quantifying gait variability, using the traditional methods (i.e. cameras and force-plates), so that reliable measures of comparison could be provided.

- Determination of variability cutoffs would be important for a proper distinction of high risk and low risk older persons.

- Evaluation of gait of older people at high and low risk of falling (as perceived by actual screening strategies) should be required. Also, abnormal gaits should be evaluated. As more variability of signals is to be expected, events detection can be more difficult to do, so that simultaneous recording with traditional techniques is required.

- When implementing the fall risk screening method on the phone, some design considerations must be taken into account, regarding the target users of application (i.e. the older persons). A clear definition of the physical test must be done, so that it can be properly executed and significant results can be obtained.

- Quantifying other movements to predict fall risk would be another important topic of research. For example, those tests related to SiSt movements would give important informations to consider, since this movement is usually performed on daily living and frequently it is a source of falls. 
- Further exploring the techniques for risk profile building should also be required. Quantification of a likelihood of falling based on some risk factors would be an important measure for fall risk prediction. Identification of risks factors that needs to be considered for this quantification, and the attribution of weights to each risk factor (which may be time variant) are important topics of research. This would also require a validation with older people at high risk and at low risk of falling.

- An evaluation of advantages and disadvantages of analysing sensors signals on the phone in contrast with using the computer should be performed. It would be important to evaluate the time that is necessary to analyse signals, and consider the possibilities to analyse signals in real time and asynchronously. 
Conclusions and future Work 


\section{References}

[1] National Institute for Health Excellence and Clinical. Clinical practice guideline for the assessment and prevention of falls in older people [CG21], volume 2. Royal College of Nursing, London, 2004.

[2] Kristina Kallin. Falls in Older People in Geriatric Care Settings: Predisposing and Precipitating Factors. PhD thesis, UmeåUniversity, 2004.

[3] World Health Organization. WHO Global report on falls Prevention in older Age. Technical report, 2007. http://www.who.int/ageing/publications/Falls_ prevention7March.pdf.

[4] Simon Conroy. Preventing falls in older people. $\mathrm{PhD}$ thesis, 2009.

[5] Mary B King. Falls, chapter 54, pages 659-669. McGraw-Hill, 6th editio edition, 2005.

[6] Michael R Narayanan, Stephen J Redmond, Maria Elena Scalzi, Stephen R Lord, Branko G Celler, and Nigel H Lovell Ast. Longitudinal falls-risk estimation using triaxial accelerometry. IEEE transactions on bio-medical engineering, 57(3):53441, March 2010.

[7] Rachel Christi King, Louis Atallah, Charence Wong, Frank Miskelly, and GuangZhong Yang. Elderly Risk Assessment of Falls with BSN. 2010 International Conference on Body Sensor Networks, pages 30-35, June 2010.

[8] X. Yu. Approaches and principles of fall detection for elderly and patient. In e-health Networking, Applications and Services, 2008. HealthCom 2008. 10th International Conference on, pages 42-47. IEEE, 2008.

[9] Ellinor Nordin. Assessment of balance control in relation to fall risk among older people. $\mathrm{PhD}$ thesis, UmeåUniversity, 2008.

[10] Michael R Narayanan, Steven R Lord, Marc M Budge, Branko G Celler, and Nigel H Lovell. Falls management: detection and prevention, using a waistmounted triaxial accelerometer. In Annual International Conference of the IEEE Engineering in Medicine and Biology Society., volume 2007, pages 4037-40, January 2007.

[11] Cliodhna Ni Scanaill, Chiara Garattini, Barry R. Greene, and Michael J. McGrath. Technology Innovation Enabling Falls Risk Assessment in a Community Setting. Ageing International, December 2010. 


\section{REFERENCES}

[12] Reto Meier. Professional Android 2 Application Development. Wiley Publishing, 2010.

[13] Maria Giné Garriga. Functional Performance in Physically Frail Community Dwelling Older Adults. PhD thesis, Universitat Ramon Llull, 2010.

[14] MJ Gibson, RO Andres, B Isaacs, T Radebaugh, and J Worm-Petersen. The prevention of falls in later life. A report of the Kellogg International Work Group on the Prevention of Falls by the Elderly. Danish medical bulletin, 34 Suppl 4:1-24, April 1987.

[15] Michael C. Nevitt, Steven R. Cummings, Sharon Kidd, and Dennis Black. Risk factors for recurrent nonsyncopal falls. A prospective study. JAMA, 261(18):2663$2668,1989$.

[16] ICECI Coordination and Maintenance Group. International Classification of External Causes of Injuries (ICECI) version 1.2 - A Related Classification in the World Health Organization Family of International Classifications. Number July. 2004.

[17] E. Rosendahl. Fall prediction and a high-intensity functional exercise programme to improve physical functions and to prevent falls among older people living in residential care facilities. PhD thesis, UmeåUniversity, 2006.

[18] Mark Speechley. Unintentional Falls in Older Adults: A Methodological Historical Review. Canadian journal on aging, 12:1-12, March 2011.

[19] Réseau Francophone de Prévention des Traumatismes et de Promotion de la Sécurité. Good Practice Guide - Prevention of falls in the elderly living at home. Éditions inpes, 2005.

[20] British Geriatics Society, American Geriatrics Society, and American Academy of Orthopaedic Surgeons Panel on Falls Prevention. Guideline for the prevention of falls in Older Persons. Journal of American Geriatrics Society, 49(5):664-672, January 2001.

[21] Sungeun Lee. Balance mechanisms during standing and walking in young and older adults. PhD thesis, University of Alberta, 2010.

[22] Miriam F Reelick, Marianne B van Iersel, Roy P C Kessels, and Marcel G M Olde Rikkert. The influence of fear of falling on gait and balance in older people. Age and ageing, 38(4):435-40, July 2009.

[23] A Hendrich, A Nyhuis, T Kippenbrock, and M E Soja. Hospital falls: development of a predictive model for clinical practice. Applied Nursing Research, 8(3):129-39, August 1995.

[24] E.Z. Riefkohl, HL Bieber, MB Burlingame, and DT Lowenthal. Medications and falls in the elderly: a review of the evidence and practical considerations. Pharmacy\&Therapeutics, 28(11):724-733, 2003.

[25] David A Ganz, Yeran Bao, Paul G Shekelle, and Laurence Z Rubenstein. Will my patient fall? JAMA : the journal of the American Medical Association, 297(1):7786, January 2007. 
[26] Afsoon Hassani Mehraban, Lynette a Mackenzie, and Julie E Byles. A self-report home environment screening tool identified older women at risk of falls. Journal of clinical epidemiology, 64(2):191-9, February 2011. http://www.ncbi.nlm.nih. gov/pubmed/20554430.

[27] The American Geriatric Society and British Geriatics Society. Falls Prevention in Older Persons - Clinical Practice Guideline. Website, 2009. http: / www . medcats . com/FALLS/ frameset.htm.

[28] CC Persad, S Cook, and B Giordani. Assessing falls in the elderly: should we use simple screening tests or a comprehensive fall risk evaluation? European journal of physical and rehabilitation medicine, 46(2):249-59, 2010.

[29] M Mancini and FB Horak. The relevance of clinical balance assessment tools to differentiate balance deficits. European journal of physical and rehabilitation medicine, 46(2):239-48, 2010.

[30] K L Perell, A Nelson, R L Goldman, S L Luther, N Prieto-Lewis, and L Z Rubenstein. Fall risk assessment measures: an analytic review. The journals of gerontology. Series A, Biological sciences and medical sciences, 56(12):M761-6, December 2001.

[31] Ivan Bautmans, Bart Jansen, Bart Van Keymolen, and Tony Mets. Reliability and clinical correlates of 3D-accelerometry based gait analysis outcomes according to age and fall-risk. Gait \& posture, January 2011.

[32] Michael Marschollek, Klaus-Hendrik Wolf, Matthias Gietzelt, Gerhard Nemitz, Hubertus Meyer zu Schwabedissen, and Reinhold Haux. Assessing elderly persons' fall risk using spectral analysis on accelerometric data-a clinical evaluation study. Annual International Conference of the IEEE Engineering in Medicine and Biology Society., pages 3682-5, January 2008.

[33] K M Culhane, M O'Connor, D Lyons, and G M Lyons. Accelerometers in rehabilitation medicine for older adults. Age and ageing, 34(6):556-60, November 2005 .

[34] R. Moe-Nilssen. A new method for evaluating motor control in gait under real-life environmental conditions. Part 2: Gait analysis. Clinical biomechanics, 13:328335, June 1998.

[35] R. Moe-Nilssen. A new method for evaluating motor control in gait under real-life environmental conditions. Part 1: The instrument. Clinical biomechanics, 13:320327, June 1998.

[36] Maura O’Sullivan, Catherine Blake, Conal Cunningham, Gerard Boyle, and Ciarán Finucane. Correlation of accelerometry with clinical balance tests in older fallers and non-fallers. Age and ageing, 38(3):308-13, May 2009.

[37] R Senden, B Grimm, I C Heyligers, H H C M Savelberg, and K Meijer. Acceleration-based gait test for healthy subjects: reliability and reference data. Gait \& posture, 30(2):192-6, August 2009. 


\section{REFERENCES}

[38] Mirko Brandes, Wiebren Zijlstra, Sander Heikens, Rob van Lummel, and Dieter Rosenbaum. Accelerometry based assessment of gait parameters in children. Gait \& posture, 24(4):482-6, December 2006.

[39] L Allet, S Armand, R a de Bie, A Golay, D Monnin, K Aminian, and E D de Bruin. Reliability of diabetic patients' gait parameters in a challenging environment. Gait \& posture, 28(4):680-6, November 2008.

[40] AMTI. AMTI Force and Motion. Website. http://www.amti.biz/.

[41] Codamotion. Codamotion: Real-Time Excellence. Website, 2010, http://www. codamotion.com/.

[42] GAITRite. GAITRite: footprints you can see. Measurements you can trust. Website. http://www.gaitrite.com/.

[43] Tekscan. MatScan $囚$ System. Website. http://www.tekscan.com/.

[44] B V McRoberts. Clinical Assessment. Website. http://www.mcroberts.nl/ products/clinical-assessment.

[45] Wiebren Zijlstra. Assessment of spatio-temporal parameters during unconstrained walking. European journal of applied physiology, 92:39-44, June 2004.

[46] William C Miller, Mark Speechley, and A Barry Deathe. Balance confidence among people with lower-limb amputations. Physical therapy, 82(9):856-65, September 2002.

[47] Barry R Greene, Alan O Donovan, Roman Romero-Ortuno, Lisa Cogan, Cliodhna Ni Scanaill, and Rose A Kenny. Quantitative Falls Risk Assessment Using the Timed Up and Go Test. IEEE Transactions on Biomedical Engineering, 57(12):2918-26, 2010.

[48] Michael R Narayanan, Maria Elena Scalzi, Stephen J Redmond, Steven R Lord, Branko G Celler, and Nigel H Lovell. A wearable triaxial accelerometry system for longitudinal assessment of falls risk. In Annual International Conference of the IEEE Engineering in Medicine and Biology Society, volume 2008, pages 2840-3, January 2008.

[49] Benoit Caby, Suzanne Kieffer, Marie de Saint Hubert, Gerald Cremer, and Benoit Macq. Feature extraction and selection for objective gait analysis and fall risk assessment by accelerometry. Biomedical engineering online, 10(1), January 2011.

[50] Jian Liu, Takehito Kanno, Masanori Akashi, Wenxi Chen, Daming Wei, Gengfeng $\mathrm{Wu}$, and Naotoshi Takeda. Patterns of Bipedal Walking on Tri-axial Acceleration Signals and Their Use in Identifying Falling Risk of Older People. The Sixth IEEE International Conference on Computer and Information Technology (CIT'06), September 2006.

[51] KM Kerr, JA White, DA Barr, and RAB Mollan. Analysis of the sit-stand-sit movement cycle in normal subjects. Clinical Biomechanics, 12(4):236-245, 1997. 
[52] Bijan Najafi, Kamiar Aminian, François Loew, Yves Blanc, and Philippe A Robert. Measurement of Stand-Sit and Sit-Stand Transitions Using a Miniature Gyroscope and Its Application in Fall Risk Evaluation in the Elderly. IEEE Transactions on Biomedical Engineering, 49(8):843-851, 2002.

[53] Niranjan Bidargaddi, Lasse Klingbeil, Antti Sarela, Justin Boyle, Vivian Cheung, Catherine Yelland, Mohanraj Karunanithi, and Len Gray. Wavelet based approach for posture transition estimation using a waist worn accelerometer. Annual International Conference of the IEEE Engineering in Medicine and Biology Society, pages 1884-7, January 2007.

[54] Daniele Giansanti. Investigation of fall-risk using a wearable device with accelerometers and rate gyroscopes. Physiological measurement, 27(11):1081-90, November 2006.

[55] Manuel Montero-Odasso, Marcelo Schapira, Enrique R Soriano, Miguel Varela, Roberto Kaplan, Luis a Camera, and L Marcelo Mayorga. Gait velocity as a single predictor of adverse events in healthy seniors aged 75 years and older. The journals of gerontology. Series A, Biological sciences and medical sciences, 60(10):1304-9, October 2005.

[56] Joe Verghese, Roee Holtzer, Richard B Lipton, and Cuiling Wang. Quantitative gait markers and incident fall risk in older adults. The journals of gerontology. Series A, Biological sciences and medical sciences, 64(8):896-901, August 2009.

[57] Jennifer S Brach, David McGurl, David Wert, Jessie M Vanswearingen, Subashan Perera, Rakie Cham, and Stephanie Studenski. Validation of a measure of smoothness of walking. The journals of gerontology. Series A, Biological sciences and medical sciences, 66(1):136-41, January 2011.

[58] Yocheved Laufer. Effect of age on characteristics of forward and backward gait at preferred and accelerated walking speed. The journals of gerontology. Series A, Biological sciences and medical sciences, 60(5):627-32, May 2005.

[59] Matthias Gietzelt, Gerhard Nemitz, Klaus-Hendrik Wolf, Hubertus Meyer Zu Schwabedissen, Reinhold Haux, and Michael Marschollek. A clinical study to assess fall risk using a single waist accelerometer. Informatics for health \& social care, 34(4):181-8, December 2009.

[60] Uffe Laessoe, Hans C Hoeck, Ole Simonsen, Thomas Sinkjaer, and Michael Voigt. Fall risk in an active elderly population-can it be assessed? Journal of negative results in biomedicine, 6(2), January 2007.

[61] K Cameron, K Hughes, and K Doughty. Reducing fall incidence in community elders by telecare using predictive systems. In 19th International Conference IEEE/EMBS Oct. 30 - Nov. 2, pages 1036-1039, 1997.

[62] Y Lajoie and SP Gallangher. Predicting falls within the elderly community: comparison of postural sway, reaction time, the Berg balance scale and the Activitiesspecific Balance Confidence (ABC) scale for comparing fallers and non-fallers. Archives of Gerontology and Geriatrics, 38(1):11-26, February 2004. 


\section{REFERENCES}

[63] Carmen Casanova Abbott. Community Multidimensional Fall Risk Screening. PhD thesis, University of Missouri, 2009.

[64] Reto W Kressig. Gait Changes in Older Adults. Website, 2009. http://www. healthandage.com/.

[65] Wataru Doyo, Rumi Kozakai, Heung-Youl Kim, Fujiko Ando, and Hiroshi Shimokata. Spatiotemporal components of the 3-D gait analysis of communitydwelling middle-aged and elderly Japanese: age- and sex-related differences. Geriatrics \& gerontology international, 11(1):39-49, January 2011.

[66] Debra J. Rose. Fallproof!: a comprehensive balance and mobility training program. Human Kinetics, 2009.

[67] Manuel Montero-Odasso, Susan W Muir, Maggie Hall, Timothy J Doherty, Marita Kloseck, Olivier Beauchet, and Mark Speechley. Gait Variability Is Associated With Frailty in Community-dwelling Older Adults. The journals of gerontology. Series A, Biological sciences and medical sciences, pages 1-9, February 2011.

[68] W Zijlstra and A L Hof. Displacement of the pelvis during human walking: experimental data and model predictions. Gait \& posture, 6(3):249-262, 1997.

[69] Wiebren Zijlstra and At L Hof. Assessment of spatio-temporal gait parameters from trunk accelerations during human walking. Gait \& posture, 18(2):1-10, October 2003.

[70] Justin J Kavanagh. Lower trunk motion and speed-dependence during walking. Journal of neuroengineering and rehabilitation, 6(9), January 2009.

[71] Hylton B Menz, Stephen R Lord, and Richard C Fitzpatrick. Acceleration patterns of the head and pelvis when walking on level and irregular surfaces. Gait \& posture, 18(1):35-46, August 2003.

[72] Rafael C González, Antonio M López, Javier Rodriguez-Uría, Diego Alvarez, and Juan C Alvarez. Real-time gait event detection for normal subjects from lower trunk accelerations. Gait \& posture, 31(3):322-5, March 2010.

[73] Diego Alvarez, Rafael C Gonzalez, Antonio Lopez, and Juan C Alvarez. Comparison of step length estimators from weareable accelerometer devices. Conference proceedings: Annual International Conference of the IEEE Engineering in Medicine and Biology Society. IEEE Engineering in Medicine and Biology Society. Conference, 1:5964-7, January 2006.

[74] Mark D Latt, Hylton B Menz, Victor S Fung, and Stephen R Lord. Acceleration patterns of the head and pelvis during gait in older people with Parkinson's disease: a comparison of fallers and nonfallers. The journals of gerontology. Series A, Biological sciences and medical sciences, 64(6):700-6, June 2009.

[75] Aner Weiss, Ilan Shimkin, Nir Giladi, and Jeffrey M Hausdorff. Automated detection of near falls: algorithm development and preliminary results. BMC research notes, 3(62), January 2010. 


\section{REFERENCES}

[76] Rolf Moe-Nilssen and Jorunn L. Helbostad. Estimation of gait cycle characteristics by trunk accelerometry. Journal of Biomechanics, 37(1):121-126, January 2004.

[77] Andrea Tura, Michele Raggi, Laura Rocchi, Andrea G Cutti, and Lorenzo Chiari. Gait symmetry and regularity in transfemoral amputees assessed by trunk accelerations. Journal of neuroengineering and rehabilitation, 7(4), January 2010.

[78] R Senden, B Grimm, I C Heyligers, H H C M Savelberg, P Willems, and K Meijer. Can an Accelerometer based Gait Test assess Fall Risk? A Comparison with the Tinetti score. 2010.

[79] B E Maki. Gait changes in older adults: predictors of falls or indicators of fear. Journal of the American Geriatrics Society, 45(3):313-20, March 1997.

[80] J M Hausdorff, H K Edelberg, S L Mitchell, a L Goldberger, and J Y Wei. Increased gait unsteadiness in community-dwelling elderly fallers. Archives of physical medicine and rehabilitation, 78(3):278-83, March 1997.

[81] J M Hausdorff, D a Rios, and H K Edelberg. Gait variability and fall risk in community-living older adults: a 1-year prospective study. Archives of physical medicine and rehabilitation, 82(8):1050-6, August 2001.

[82] D L Streiner. Maintaining standards: differences between the standard deviation and standard error, and when to use each. Canadian journal of psychiatry, 41(8):498-502, October 1996.

[83] Hylton B Menz, Stephen R Lord, and Richard C Fitzpatrick. Acceleration patterns of the head and pelvis when walking are associated with risk of falling in community-dwelling older people. The journals of gerontology. Series A, Biological sciences and medical sciences, 58(5):M446-52, May 2003.

[84] J.G.T. Ribeiro, J.T.P. De Castro, and J.L.F. Freire. Using the FFT-DDI method to measure diplacements with piezoelectric, resistive and ICP accelerometers. In IMAC-XXI: A Conference \& Exposition on Structural Dynamics; Kissimmee, FL. Society for Experimental Mechanics, 2003.

[85] L.D. Slifka. An Accelerometer based approach to measuring displacement of a vehicle body. PhD thesis, Horace Rackham School Of Graduate Studies of the University of Michigan, 2004.

[86] G Sometimes. A Textbook Chapter on Integrating Discrete Functions. Website. http://numericalmethods.eng.usf.edu/topics/discrete_data_ integration.html.

[87] Android Developers. Android Developers. Website. http://developer.android. com/index.html.

[88] Ed Burnette. Hello Android - Introducing Google's Mobile Development Platform. 3 th editio edition, 2010.

[89] Wilfried Elmenreich. Sensor Fusion in Time-Triggered Systems. PhD thesis, O. Univ.-Prof. Dr. Hermann Kopetz, 2002. 


\section{REFERENCES}

[90] Joy Goodman, Stephen Brewster, and Philip Gray. Older people, mobile devices and navigation. In Proceedings of the Workshop on HCI and the Older Population, pages 13-14, 2004.

[91] Suziah Sulaiman and Intan Suriany Sohaimi. An Investigation to Obtain a Simple Mobile Phone Interface for Older Adults. In 2010 International Conference on Intelligent and Advanced Systems (ICIAS), 2010.

[92] Isabel Seruca, José Cordeiro, and Slimane Hammoudi. Enterprise information systems VI. Springer, 2006.

[93] Mary Zajicek. Passing on Good Practice: Interface Design for Older Users. In Computer Human Interaction, pages 636-640. Springer, 2004.

[94] Google. Nexus S - The new Android phone from Google. Website, 2011. http: //www.google.com/nexus/.

[95] MathWorks. MathWorks - MATLAB and Simulink for Technical Computing. Website, 2011. http://www . mathworks.com/.

[96] Ge Wu and Peter R Cavanagh. ISB Recommendations in the Reporting for Standardization of Kinematic Data. Journal of Biomechanics, 28(10):1257-1261, 1995.

[97] Barry Bogin and Maria Inês Varela-Silva. Leg length, body proportion, and health: a review with a note on beauty. International journal of environmental research and public health, 7(3):1047-75, March 2010.

[98] Victorian Government Health Information. Falls Risk for Older People - Community setting (FROP-Com). Website, 2005. http://www.health.vic.gov.au/.

[99] Keith Hill. Activities-specific and Balance Confidence (ABC) Scale. Australian Journal Of Physiotherapy, 51(3):197, 2005.

[100] Meredith Wallace and Mary Shelkey. Katz Index of Independence in Activities of Daily Living. Assisted Living Consult, pages 21-2, February 2008.

[101] Tex Texin. International Shoe Size Conversion Charts. Website, 2010. http: //www.il8nguy.com/l10n/shoes.html.

[102] S. Fioretti, M. Maurizi, and D. Tronelli. Kinematic and Dynamic Analysis of the Functional Reach Test in Elderly Subjects using Force-Plate Measurements. In Proceedings of the 22nd Annual EMBS International Conference, pages 18691872, 2000.

[103] P A Stalenhoef, J P M Diederiks, J A Knottnerus, A D M Kester, and H F J M Crebolder. A risk model for the prediction of recurrent falls in community-dwelling elderly: A prospective cohort study. Journal of Clinical Epidemiology, 55:10881094, 2002.

[104] W Dite and V Temple. A clinical test of stepping and change of direction to identify multiple falling older adults. Archives of Physical Medicine and Rehabilitation, 83(11):1566-1571, November 2002. 


\section{REFERENCES}

[105] Ellinor Nordin, Nina Lindelö, Erik Rosendahl, Jane Jensen, and Lillemor LundinOlsson. Prognostic validity of the Timed Up-and-Go test, a modified Get-Upand-Go test, staff's global judgement and fall history in evaluating fall risk in residential care facilities. Age and Ageing, 37(May):442-448, 2008.

[106] Ellinor Nordin, Erik Rosendahl, and Lillemor Lundin-Olsson. Timed "Up \& Go" Test: Reliability in Older People Dependent in Activities of Daily Living — Focus on Cognitive State. Physical Therapy, 86(5):646-655, 2006.

[107] A Khasnis and R M Gokula. Romberg's test. Journal of postgraduate medicine, 49(2):169-72, 2003.

[108] Janet M. Simpson, Chris Worsfold, Elizabeth Reilly, and Natalie Nye. A standard procedure for using TURN 180: Testing dynamic postural stability among elderly people. Physiotherapy, 88(6):342-353.

[109] L D Bogle Thorbahn and R a Newton. Use of the Berg Balance Test to predict falls in elderly persons. Physical therapy, 76(6):576-83, June 1996.

[110] Sadler Physical Therapy Services. Berg Balance Scale. Website, 2007. http: //sadlerpt.com/SPTS/Resources_1_files/LongForm.pdf.

[111] Sadler Physical Therapy Services. Dynamic Gait Index Score Sheet. Website, 2007. http://sadlerpt.com/SPTS/Resources_1_files/DynamicGaitIndex.pdf.

[112] FB Horak, DM Wrisley, and J Frank. The Balance Evaluation Systems Test (BESTest) to Differentiate Balance Deficits. Physical Therapy, 89(5):484-498, 2009.

[113] FB Horak and DM Wrisley. Balance Evaluation Systems Test (BESTest) - eAppendix. Physical Therapy, 89(5):eAppendix, 2009.

[114] Stephen R Lord, Hylton B Menz, and Anne Tiedemann. A physiological profile approach to falls risk assessment and prevention. Physical therapy, 83(3):237-52, March 2003.

[115] Falls Group and Balance Research. Fallscreen - the falls risk calculator. Website. http://www. neura.edu.au/fbrg.

[116] Janine Hatch, Kathleen M Gill-Body, and Leslie G Portney. Determinants of balance confidence in community-dwelling elderly people. Physical therapy, 83(12):1072-9, December 2003.

[117] Pacific Balance \& Dizziness Center. Activity-specific Balance Confidence Scale. Website, 2011. http://www.pacificbalancecenter.com/downloads.php.

[118] M E Tinetti, D Richman, and L Powell. Falls efficacy as a measure of fear of falling. Journal of gerontology, 45(6):P239-43, November 1990.

[119] Fall Prevention Task Force. Common Screening And Assessment Tools. Website. http://www.fallpreventiontaskforce.org/tools.htm. 


\section{REFERENCES}

[120] K D Hill, J a Schwarz, a J Kalogeropoulos, and S J Gibson. Fear of falling revisited. Archives of physical medicine and rehabilitation, 77(10):1025-9, October 1996.

[121] Nancy Edwards and Donna Lockett. Development and validation of a modified falls-efficacy scale. Disability \& Rehabilitation: Assistive Technology, 3(4):193200, 2008.

[122] Department of Human Services Victoria Australia. Modified Falls Efficacy Scale Health and well-being. Website, 2009. http://www.health.vic.gov.au/.

[123] A M Jette, A R Davies, P D Cleary, D R Calkins, L V Rubenstein, A Fink, J Kosecoff, R T Young, R H Brook, and T L Delbanco. The Functional Status Questionnaire: reliability and validity when used in primary care. Journal of general internal medicine, 1(3):143-9, 1986.

[124] Alan M. Jette, Allyson R. Davies, Paul D. Cleary, David R. Calkins, Lisa V. Rubenstein, Arlene Fink, Jacqueline Kosecoff, Roy T. Young, Robert H. Brook, and Thomas L. Delbanco. Erratum: The functional status questionnaire: Reliability and validity when used in primary care. Journal of General Internal Medicine, 1(6):427-427, November 1986.

[125] Society of Hospital Medicine. Society of Hospital Medicine. Website, 2011. http: //www.hospitalmedicine.org/.

[126] P. Gompertz, P. Pound, and S. Ebrahim. A postal version of the Barthel Index. Clinical Rehabilitation, 8(3):233-239, August 1994.

[127] Irene Hartigan and Denis O'Mahony. The Barthel Index: comparing inter-rater reliability between nurses and doctors in an older adult rehabilitation unit. Applied nursing research, 24(1):e1-7, February 2011.

[128] Internet Stroke Center. Stroke Scales \& Clinical Assessment Tools. Website, 2010. http://www.strokecenter.org/trials/scales/.

[129] C. Graf. The Lawton Instrumental Activities of Daily Living Scale. American Journal of Nursing, 108(4):52-62, August 2008.

[130] The University of Newcastle. Women's Health Australia (WHA). Website, 2011. http://www.alswh.org.au/. 


\section{Appendix A}

\section{Screening tools}

A complete description of screening tools is here presented.

\section{A.1 Single Task Performance Measures}

\section{A.1.1 One-legged stand (OLS)}

The OLS aims to assess the postural stability. It doesn't require specific equipment, and its administration varies across settings, including which leg is selected, if the eyes are open or closed, the total time recorded and the number of repetitions. However, there is some doubt about its predictability of future falls, since some studies found no relation between OLS time and future falls risk [28].

\section{A.1.2 Functional Reach Test (FR)}

The FR is a clinical measure of balance. Specifically, this test measures the maximum forward displacement that a subject can give to his or her hand, maintaining a $90^{\circ}$ flexion in the shoulder and without changing the base of support or making a step, as illustrated in Figure A.1 [102].

The test is scored as $0,1,2$ or 3 if the individual is not able to reach, reaches 1 to 15 $\mathrm{cm}, 16$ to $25 \mathrm{~cm}$ or $26 \mathrm{~cm}$ or more, respectively [103].

This test was developed to evaluate the maximum limits of stability in stance [29]. Reaching in the lateral and backwards directions have also been added [29].

\section{A.1.3 Sit-to-stand transfer with 5 repetitions (STS5)}

This test begins in a seated position, following by five SiSt transfers with the arms folded (illustrated on Figure A.2), as quickly as possible. The time to complete all movements is the performance metrics of this test [10]. The movements of STS5 are crucial for an independent living and their performance is related to balance and mobility, which are related with lower limb strength, postural sway and proprioception [6].

\section{A.1.4 Rapid Step Test (RST)}

The individual is instructed to take a step as quickly as possible and then return to the starting position. This test was not found to be a good predictor of falls [28]. 

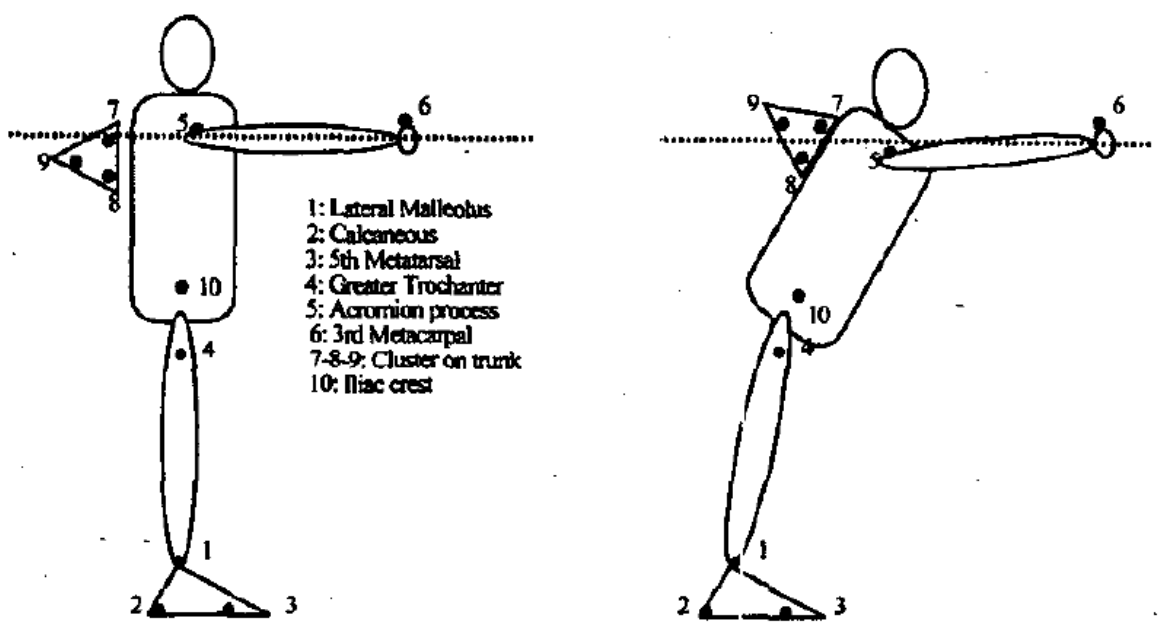

Figure A.1: Illustration of the Functional Reach Test. Source: [102].

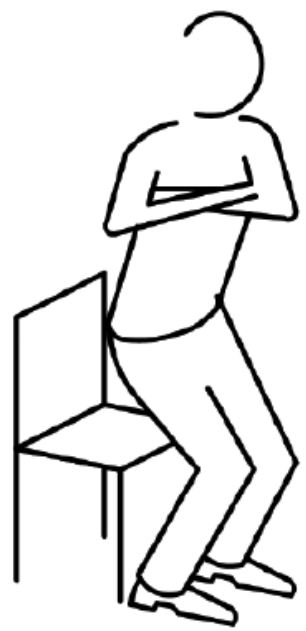

Figure A.2: Illustration of the STS5 execution. Source: [6].

\section{A.1.5 Alternative Step Test (AST)}

As denoted by its name, this test implies the alternatively placing of the whole left and right foot eight times as fast as possible onto and off a platform $(19 \mathrm{~cm}$ high and $40 \mathrm{~cm}$ wide), as illustrated on Figure A.3. The time taken to complete the task is the measured outcome $[6,10]$.

\section{A.1.6 Four square step test (FSST)}

The individual is asked to rapidly change direction in a prescribed order maintaining the face forward during the entire sequence in four square blocks so that a step forward, 


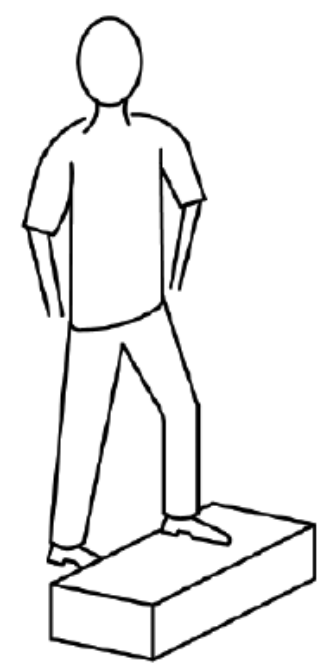

Figure A.3: Illustration of the AST execution. Source: [6].

backward and side-ways is performed [104, 28]. An example of a set-up of this test is provided in Figure A.4.

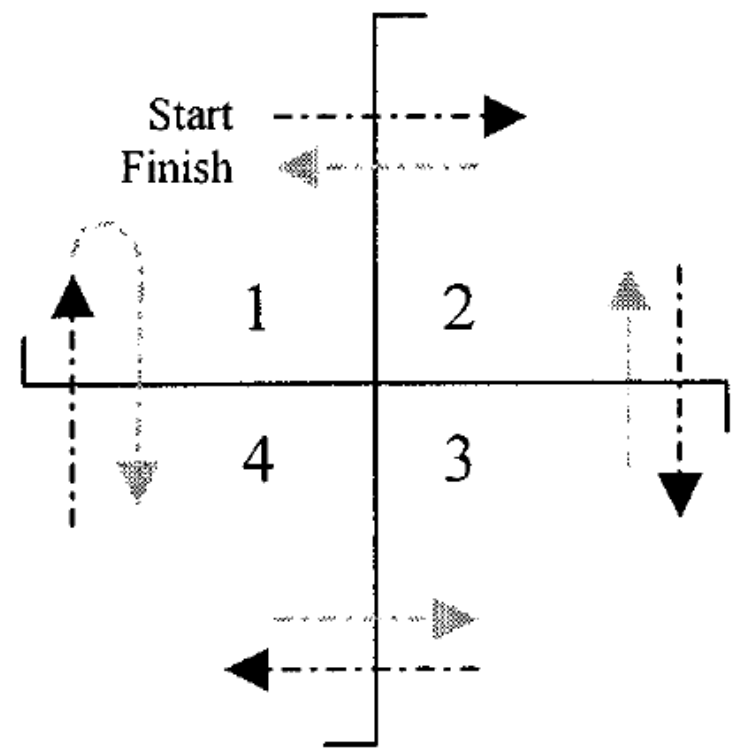

Figure A.4: An example of a FSST setup. The subject starts in square 1, facing the square 2. Then, he/she performs the following sequential movements: step forward into square 2, sideway to 3 , backward to 4, sideway to 1 , sideway to 4 , forward to 3, sideway to 2 and finally backward to 1 . Source: [104].

The time to complete the test is the outcome measured in this test [104]. 


\section{A.1.7 Maximal Step Length (MSL)}

Patients are instructed to keep their arms crossed on their chests while stepping out with one leg as far as they can while maintaining their position with their other leg. Then, the individual has to return to the initial position in one step. The test is repeated five times in each direction [28].

\section{A.1.8 Comfortable Gait Speed (CGS)}

CGS enables to measure the dynamic balance. It consists of walking over a relatively short distance at a usual speed of walking. The outcome of this test is the time necessary to complete it. More than 5 seconds to walk a 4 meter distance is considered a high risk of falling [28].

\section{A.1.9 Timed Up \& Go Test (TUGT)}

The TUGT is a simple and inexpensive method for assessing future falls risk in older people. As illustrated in Figure A.5, the TUGT consists of getting up from a chair, walking 3 meters at a normal pace, turning around at a designated spot (marked with a red " $x$ " on Figure A.5), returning to the chair and sitting down [7, 47]. The movements included in TUGT impose demands on the balance control system, including transitions from SiSt and $\mathrm{StSi}$, as well as acceleration and deceleration phases of gait, and directional changes of head and body [9].

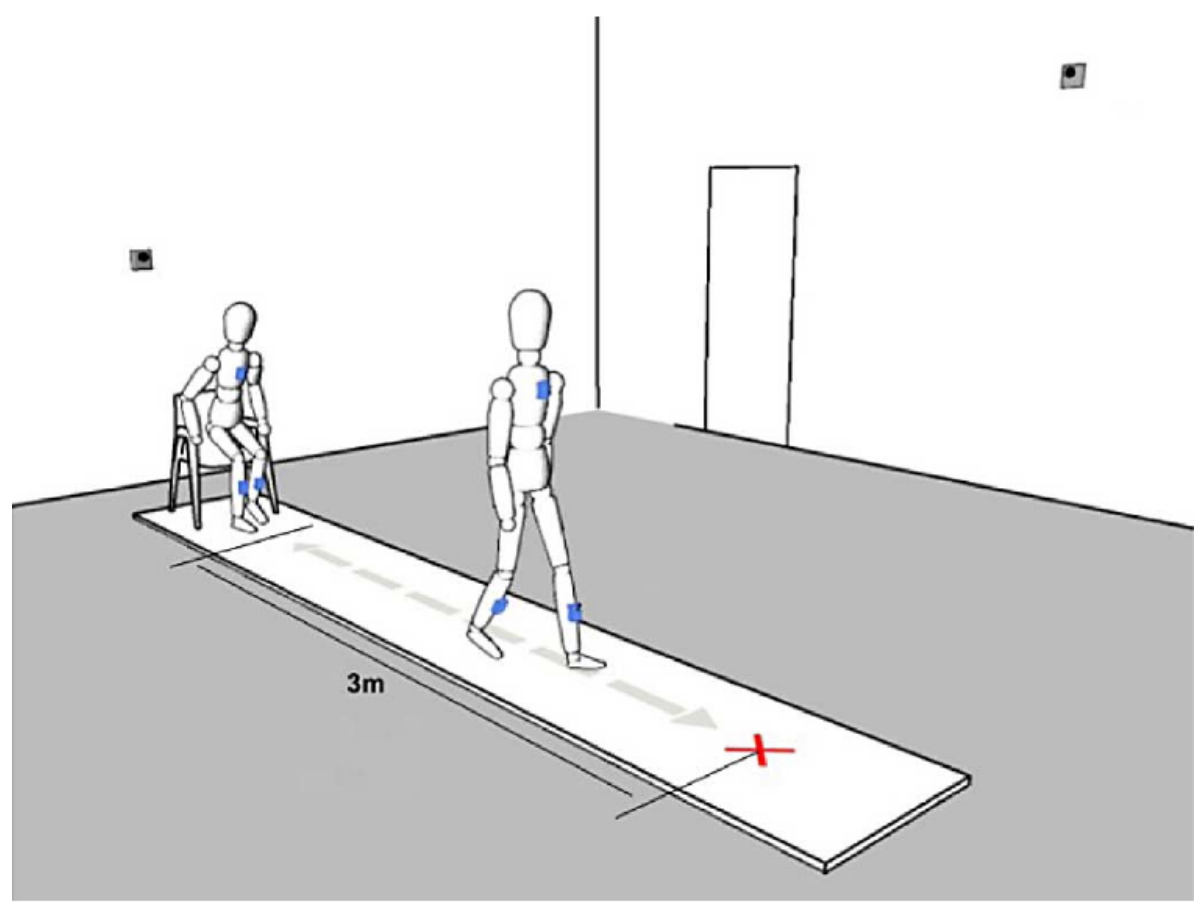

Figure A.5: Illustration of the TUGT. Source: [47].

The time to complete this sequence is indicative of the likelihood of falling, since older persons with longer TUGT times are more likely to fall [47]. Usually, older people without balance problems can perform the test within 10-25 seconds. Above this threshold, 
the individual is assumed to be at high risk [105] and should be considered for a complete fall risk assessment [1].

The test is recommended by the British Geriatrics Society, the American Geriatrics Society, and Nordic geriatricians to screen the risk of falling in older people $[9,106]$. It is usually used in geriatric clinics by clinicians or physiotherapists [47].

However, this tool admits always some variability, which depends on the person being measured or the person who does the measuring. In clinical assessments, professionals should be aware that the patient's true value can be above or below the measured one, within an expected range [106].

Quantitative assessment of gait and turning during the TUGT should allow for more objective and sensitive determination of falls risk [47].

\section{A.1.10 Romberg's Test}

In this test, the patient stands with his feet close together, arms by the side and eyes open. If any significant swaying or tendency to fall occurs, it should be noted. Then, the person is asked to close his/her eyes. The postural sway is again noted and compared with the open eyes experiment [107].

An higher risk of falling is denoted if there is significant imbalance with the eyes closed, or if the imbalance makes significantly worse when the individual close his/her eyes [107].

\section{A.1.11 Near-tandem standing balance (NTSB)}

This test consists of standing in the near-tandem position for 30 seconds with the eyes closed. The near-tandem position implies the laterally separation of feet by $2.5 \mathrm{~cm}$ and heel of the front $2.5 \mathrm{~cm}$ anterior to the large toe of the back foot. The performance metrics for this test is the lateral sway or the occurrence of a protective step [10].

\section{A.1.12 Turn $180^{\circ}$}

This tool is used to identify dynamic postural stability, allowing a quick identification of the severity of problems in people who have difficulty in maintaining a stable position. It can be performed in people's homes or in clinics and hospitals [108].

During the test, the person performs a complete $180^{\circ}$ turn and the number of steps required to do that are accounted. If more than four steps are required to perform this task, the person is identified at high risk of falling [108].

\section{A.2 Multi-task Measures}

\section{A.2.1 Performance-Oriented Mobility Assessment (POMA)}

POMA, also called Tinetti Gait and Balance Assessment, assesses both balance and gait through the direct observation of tasks [28], Figure A.6.

It is used to assess the risk of falling of older people and is performed by trained personnel. Each section (balance and gait) has a score based on different observations, which added together can give a final score of 28 . The patient is classified as "low risk" 
Screening tools

\begin{tabular}{|c|c|c|}
\hline Activity & Patient observations & Score \\
\hline \multicolumn{3}{|l|}{ Balance } \\
\hline $\begin{array}{l}\text { Sitting } \\
\text { Balance }\end{array}$ & $\begin{array}{l}\text { Is there leaning or sliding in the chair or is } \\
\text { the patient steady and safe? }\end{array}$ & $0-1$ \\
\hline $\begin{array}{l}\text { Rises from } \\
\text { chair }\end{array}$ & $\begin{array}{l}\text { Can the patient rise without assistance or } \\
\text { the use or arms? }\end{array}$ & $0-2$ \\
\hline $\begin{array}{l}\text { Attempts to } \\
\text { rise }\end{array}$ & $\begin{array}{l}\text { Can the patient rise without assistance and } \\
\text { are multiple attempts required? }\end{array}$ & $0-2$ \\
\hline $\begin{array}{l}\text { Immediate } \\
\text { standing } \\
\text { balance }\end{array}$ & $\begin{array}{l}\text { Is the patient unsteady, steady but uses a } \\
\text { walking aid, or independently steady? }\end{array}$ & $0-2$ \\
\hline $\begin{array}{l}\text { Standing } \\
\text { balance }\end{array}$ & $\begin{array}{l}\text { Is the patient unsteady, steady with a } \\
\text { walker or with a wide stance, or steady } \\
\text { with a narrow stance? }\end{array}$ & $0-2$ \\
\hline Nudge & $\begin{array}{l}\text { When nudged does the patient begin to } \\
\text { fall; stagger, grab or self catch; or is } \\
\text { steady? }\end{array}$ & $0-2$ \\
\hline Eyes closed & Is the patient steady with eyes closed & $0-1$ \\
\hline Turing $360^{\circ}$ & $\begin{array}{l}\text { When turning are the steps continuous and } \\
\text { is the patient steady? }\end{array}$ & $0-2$ \\
\hline Sitting down & $\begin{array}{l}\text { Is the patient safe sitting down; use the } \\
\text { chair arms; or is the patient unsafe? }\end{array}$ & $0-2$ \\
\hline \multicolumn{3}{|c|}{ ( } \\
\hline $\begin{array}{l}\text { Indication of } \\
\text { gait }\end{array}$ & $\begin{array}{l}\text { Is there any hesitancy when starting to } \\
\text { walk? }\end{array}$ & $0-1$ \\
\hline $\begin{array}{l}\text { Step length } \\
\text { and height }\end{array}$ & $\begin{array}{l}\text { Do the right and left feet pass the other } \\
\text { foot with each step? }\end{array}$ & $0-2$ \\
\hline $\begin{array}{l}\text { Foot } \\
\text { clearance }\end{array}$ & Do the right and left feet clear the floor? & $0-2$ \\
\hline $\begin{array}{l}\text { Step } \\
\text { symmetry }\end{array}$ & Are the right and left step equal in length? & $0-1$ \\
\hline $\begin{array}{l}\text { Step } \\
\text { continuity }\end{array}$ & Are the steps continuous? & $0-1$ \\
\hline Path & $\begin{array}{l}\text { Is there any deviation in the path taken, is } \\
\text { a walking aid used, or is the path straight } \\
\text { without an aid? }\end{array}$ & $0-2$ \\
\hline Trunk & $\begin{array}{l}\text { Is there sway of the trunk, is a walking aid } \\
\text { used, is there any flexion of the trunk, or } \\
\text { no sway or flexion without using an aid? }\end{array}$ & $0-2$ \\
\hline $\begin{array}{l}\text { Walking } \\
\text { time }\end{array}$ & $\begin{array}{l}\text { Are heals apart or almost touching while } \\
\text { walking? }\end{array}$ & $0-1$ \\
\hline
\end{tabular}

Figure A.6: Performance-Oriented Mobility Assessment. Source: [7].

if the final score is 18 or under, "medium risk" if the final score is comprised between 19 and 23 and "high risk" if it scores 24 or over [7].

This scale has the advantage of include both balance and gait, and has a good interrater reliability and sensitivity [29].

\section{A.2.2 Berg Balance Scale (BBS)}

The BBS consists of 14 separate items (see Figure A.7), graded on a scale of 0-4. A maximum score of 56 can be obtained and a score below 45 is indicative of high risk of 
falling [29, 109]. To complete the test, no specific instruments are necessary. A chair with and without arms, a tape measure, a step stool and a stopwatch may be enough [109].

\begin{tabular}{|cl|}
\hline Item & Description \\
\hline 1 & Sitting to standing \\
2 & Standing unsupported \\
3 & Sitting unsupported \\
4 & Standing to sitting \\
5 & Transfers \\
6 & Standing with eyes closed \\
7 & Standing with feet together \\
& Reaching forward with an \\
8 & outstretched arm \\
9 & Retrieving object from floor \\
10 & Turning to look behind \\
11 & Turning 360 \\
12 & Placing alternate foot on stool \\
13 & Standing with one foot in front of \\
14 & the other foot \\
\hline
\end{tabular}

Figure A.7: The Berg Balance Test Items. Source: [109].

The complete BBS can be found in [110].

\section{A.2.3 Dynamic Gait Index (DGI)}

The DGI evaluates alterations in gait in response to eight different tests. Each one can be scored from 0 to 3, and, after summing each one a total score of 0 to 24 can be obtained [28, 111]. A total score below 21 indicates risk for falls [111].

As can be seen in [111], the DGI evaluates gait at normal speed, changes in gait speed, horizontal and vertical head turns, body turn, stepping over and around obstacles and walk up stairs.

\section{A.2.4 Balance Evaluation Systems Test (BESTest)}

The BESTest consists of 36 items, grouped into 6 systems underlining balance control, as represented on Figure A.8 [29, 112].

Several tests from each system are performed, and a score from 0 to 3 is attributed to each one [112]. The tests are enumerated on Figure A.9 and a complete description of this tool is presented in [113].

\section{A.2.5 Physiological Profile Assessment (PPA)}

The PPA is a valid and reliable measure of fall risk in older people [29]. It is organized around the physiologic impairments that lead to fall risk and involves a series of five simple tests of vision, tactile sensitivity, leg muscle force, reaction time, and postural sway in stance. The PPA has two versions: a comprehensive (long) version and a screening (short) version [114].

An illustration of the short from is provided in Figure A.10.

A complete description of PPA long version is provided in [114]. 


\section{Screening tools}

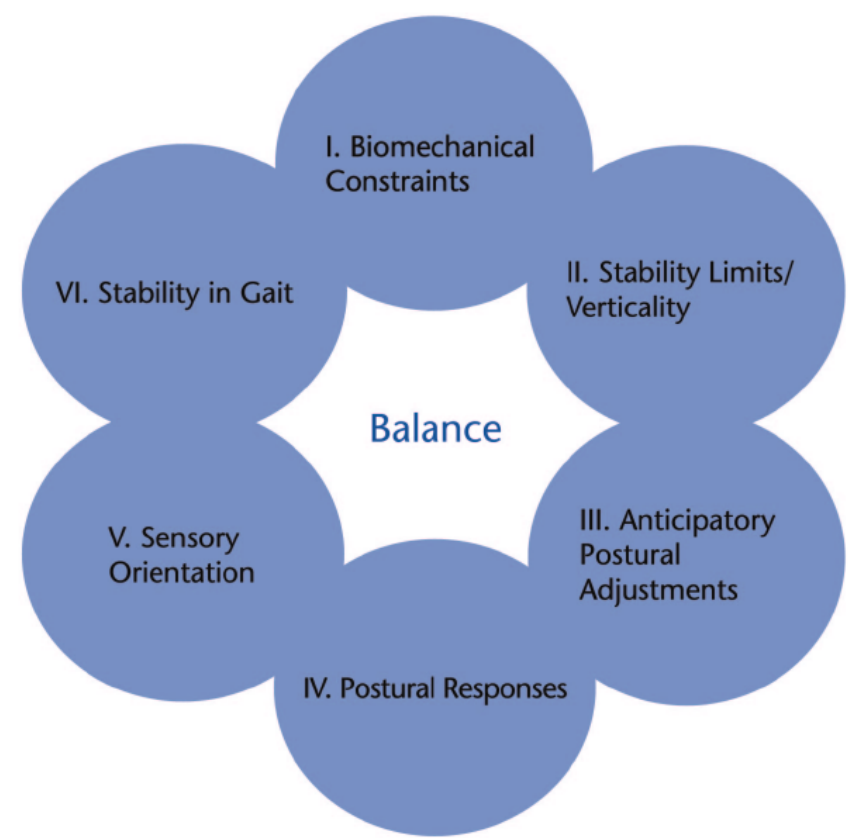

Figure A.8: The six systems/sections evaluated on BESTest. Source: [112].

\begin{tabular}{|c|c|c|c|c|c|}
\hline $\begin{array}{l}\text { I. Biomechanical } \\
\text { Constraints }\end{array}$ & $\begin{array}{c}\text { II. Stability } \\
\text { Limits/Verticality }\end{array}$ & $\begin{array}{l}\text { III. Anticipatory } \\
\text { Postural } \\
\text { Adjustments }\end{array}$ & $\begin{array}{l}\text { IV. Postural } \\
\text { Responses }\end{array}$ & $\begin{array}{l}\text { v. Sensory } \\
\text { Orientation }\end{array}$ & VI. Stability in Gait \\
\hline 1. Base of support & $\begin{array}{l}\text { 6. Sitting } \\
\text { verticality (left } \\
\text { and right) and } \\
\text { lateral lean (left } \\
\text { and right) }\end{array}$ & 9. Sit to stand & $\begin{array}{l}\text { 14. In-place } \\
\text { response, forward }\end{array}$ & \multirow{3}{*}{$\begin{array}{l}\text { 19. Sensory integration } \\
\text { for balance } \\
\text { (modified CTSIB) } \\
\text { Stance on firm } \\
\text { surface, EO } \\
\text { Stance on firm } \\
\text { surface, EC } \\
\text { Stance on foam, EO } \\
\text { Stance on foam, EC }\end{array}$} & $\begin{array}{l}\text { 21. Gait, level } \\
\text { surface }\end{array}$ \\
\hline 2. CoM alignment & $\begin{array}{l}\text { 7. Functional } \\
\text { reach forward }\end{array}$ & 10. Rise to toes & $\begin{array}{l}\text { 15. In-place } \\
\text { response, } \\
\text { backward }\end{array}$ & & $\begin{array}{l}\text { 22. Change in gait } \\
\text { speed }\end{array}$ \\
\hline $\begin{array}{l}\text { 3. Ankle strength } \\
\text { and ROM }\end{array}$ & $\begin{array}{l}\text { 8. Functional } \\
\text { reach lateral } \\
\text { (left and right) }\end{array}$ & $\begin{array}{l}\text { 11. Stand on one } \\
\text { leg (left and } \\
\text { right) }\end{array}$ & $\begin{array}{l}\text { 16. Compensatory } \\
\text { stepping } \\
\text { correction, } \\
\text { forward }\end{array}$ & & $\begin{array}{l}\text { 23. Walk with head } \\
\text { turns, horizontal }\end{array}$ \\
\hline $\begin{array}{l}\text { 4. Hip/trunk lateral } \\
\text { strength }\end{array}$ & & $\begin{array}{l}\text { 12. Alternate stair } \\
\text { touching }\end{array}$ & $\begin{array}{l}\text { 17. Compensatory } \\
\text { stepping } \\
\text { correction, } \\
\text { backward }\end{array}$ & 20. Incline, EC & $\begin{array}{l}\text { 24. Walk with pivot } \\
\text { turns }\end{array}$ \\
\hline \multirow[t]{3}{*}{$\begin{array}{l}\text { 5. Sit on floor and } \\
\text { stand up }\end{array}$} & & $\begin{array}{l}\text { 13. Standing arm } \\
\text { raise }\end{array}$ & $\begin{array}{l}\text { 18. Compensatory } \\
\text { stepping } \\
\text { correction, lateral } \\
\text { (left and right) }\end{array}$ & & $\begin{array}{l}\text { 25. Step over } \\
\text { obstacles }\end{array}$ \\
\hline & & & & & $\begin{array}{l}\text { 26. Timed "Get Up } \\
\text { \& Go" Test }\end{array}$ \\
\hline & & & & & $\begin{array}{l}\text { 27. Timed "Get Up } \\
\& \text { Go" Test with } \\
\text { dual task }\end{array}$ \\
\hline
\end{tabular}

${ }^{a} \mathrm{CoM}=$ center of mass, $\mathrm{ROM}=$ range of motion, $\mathrm{CTSIB}=$ Clinical Test of Sensory Integration for Balance, EO=eyes open, $\mathrm{EC}=$ eyes closed.

Figure A.9: Summary of tests performed under each system category of BESTest. Source: [112]. 


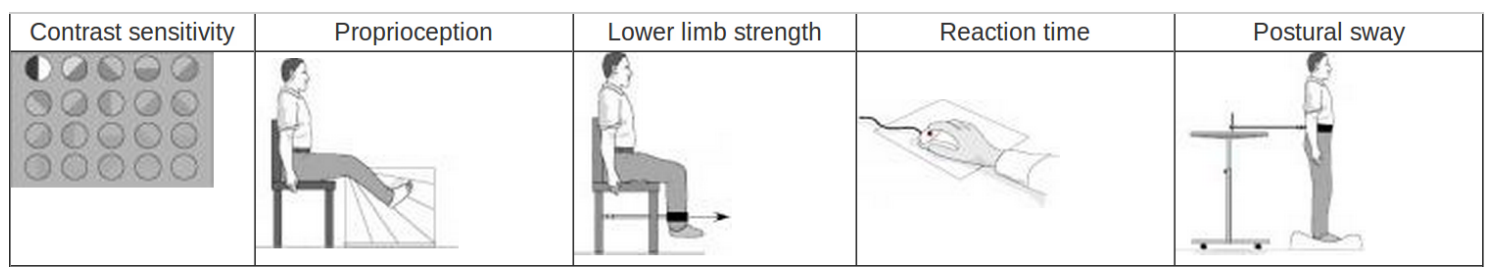

Figure A.10: PPA short-form tests. Source: [115].

\section{A.3 Self-reported tools}

\section{A.3.1 The Falls Risk for Older People in the Community scale (FROP-Com)}

This tool consists of 13 rated risk factors, which are history of falls, medications, medical conditions, sensory loss, feet $\&$ footwear, cognitive status, continence, nutritional status, environment, functional behaviour, function, balance, gait/physical activity. The total score can indicate low falls risk $(0-15)$, mild to moderate falls risk $(16-24)$ and high falls risk $(>24)$. The complete tool is available on-line on [98].

The FROP-Com can be classified as a clinician-rated scale and consists of a structured questionnaire that doesn't require any special equipment [28].

\section{A.3.2 Activities-Specific Balance Confidence Scale (ABCS)}

The ABCS is a 16-item questionnaire used to quantify the balance confidence, i.e. the individual's perceived capabilities in balance function. It is based on the performance of situation-specific activities, such as climbing stairs, reaching above the head and walking on different surfaces. Each item is scored from $0 \%$ (no confidence) to $100 \%$ (full confidence in the ability to perform the activity without loosing balance or becoming unsteady) and the total score is the averaged sum of the individual responses [116].

ABCS has good test-retest reliability $(r=0.92)$ [99, 116]. A cut-off score of $67 \%$ resulted in $84 \%$ sensitivity and $87 \%$ specificity in correctly classifying fallers and nonfallers in a cross-sectional study of older people living in the community [99].

ABSC have been found to be able to discriminate between fearful and nonfearful subjects and those who avoided activity due to fear of falling from those who did not [99, 116]. Lower ABSC scores were associated with lower levels of activity and falls [99].

ABSC has been shown to have better scale responsiveness than the FES when used in community-dwelling elderly people.

The administration of this scale require that the individual has reasonably intact cognition, so that he or she can understand that what is being assessed is confidence in doing the activity, not ease with which the activity can be performed [99].

The study from [116] demonstrated that the TUGT scores are highly correlated with ABCS scores, which demonstrates the existence of a relationship between balance confidence and functional mobility. 


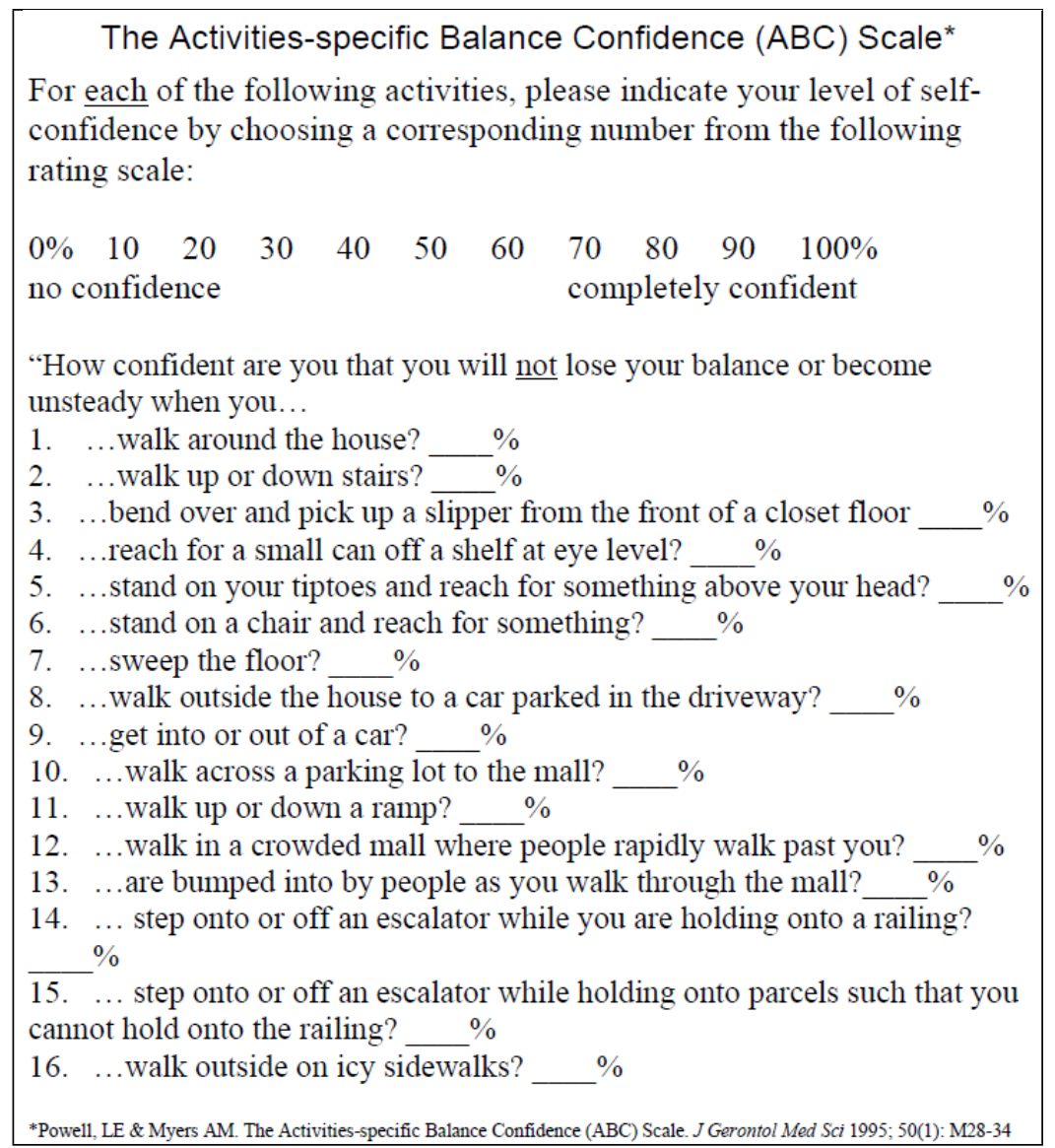

Figure A.11: Activities-Specific Balance Confidence Scale (ABCS). Available on: [117].

\section{A.3.3 Falls Efficacy Scale (FES)}

The Falls Efficacy Scale was developed by [118] to measure fear of falling, considering its definition as "low perceived self-efficacy at avoiding falls during essential, non-hazardous activities of daily living".

It is a 10-item questionnaire that can be either self-administered or administered by interview. It asks their respondents to rate their level of confidence, from 1 (extreme confident) to 10 (no confidence at all) in performing common activities, such as taking a shower, getting dressed or reaching into cabinets without falling [116].

The higher scores then correspond to subjects with low self-efficacy or confidence, representing subjects with fear of falling.

FES showed good test-retest reliability (Pearson's correlation 0.71) in two samples of community-living elderly persons, and appears to be a reliable and valid method for measuring fear of falling [118].

\section{A.3.4 Modified Falls Efficacy Scale (MFES)}

The MFES is a modified version of FES, developed by [120], that includes additional items related to the confidence in performing outdoor activities, comprising a total of 14 items. 


\begin{tabular}{|c|c|}
\hline Activity & $\begin{array}{l}\text { Score } \\
1 \text { very confident } \\
10 \text { not confident at all }\end{array}$ \\
\hline \multicolumn{2}{|l|}{ Take a bath or shower } \\
\hline \multicolumn{2}{|l|}{ Reach into cabinets or closets } \\
\hline \multicolumn{2}{|l|}{ Walk around the house } \\
\hline \multicolumn{2}{|l|}{$\begin{array}{l}\text { Prepare meals not requiring carrying } \\
\text { heavy or hot objects }\end{array}$} \\
\hline \multicolumn{2}{|l|}{ Get in and out of bed } \\
\hline \multicolumn{2}{|l|}{ Answer the door or telephone } \\
\hline \multicolumn{2}{|l|}{ Get in and out of a chair } \\
\hline \multicolumn{2}{|l|}{ Getting dressed and undressed } \\
\hline \multicolumn{2}{|l|}{$\begin{array}{l}\text { Personal grooming (i.e. washing } \\
\text { your face) }\end{array}$} \\
\hline \multicolumn{2}{|l|}{ Getting on and off of the toilet } \\
\hline Total Sco & \\
\hline
\end{tabular}

Figure A.12: Falls Efficacy Scale (FES). Available on: [119].

It also measures fear of falling based on the concept of self-efficacy [116]. However, the MFES demonstrated greater internal consistency and better response variability than the original FES in a cohort of community-living seniors [121]. It also presents high retest reliability, according to [120].

\section{A.3.5 Functional Status Questionnaire (FSQ)}

FSQ was designed to screen for disability and to monitor changes in physical, psychological, social and role functions. It is a brief questionnaire, which can be self-administered in about 15 minutes [123].

Six summary scale scores (i.e. basic ADL, intermediate ADL, mental health, work performance, social activity and quality of interaction) and six single-item questions produce a total of 34 items [123].

Each scale score is transformed to a range from 0 to 100 (Equation A.1), where higher scores means better functional ability.

$$
S S=\frac{\sum_{i=1}^{n} y_{i}-n}{n} \frac{100}{k}
$$

where $S S$ is the transformed FSQ scale score, $y_{1}$ are the individual questionnaire response score, $n$ is the number of questions for which valid information is available and $k$ is the maximum minus minimum valid response score $[123,124]$. $k$ is set to 3 for basic ADL, intermediate ADL, work performance and social activities, and 5 to other scales [125].

A warning zone exists to help clinicians interpret individual FSQ scores and identifying specific problem areas. This zone represent important functional disabilities that need to be investigated more [123].

On the next Table A.1, warning zones are identified.

According to [123], FSQ produces reliable sub-scales with construct validity. 
Screening tools

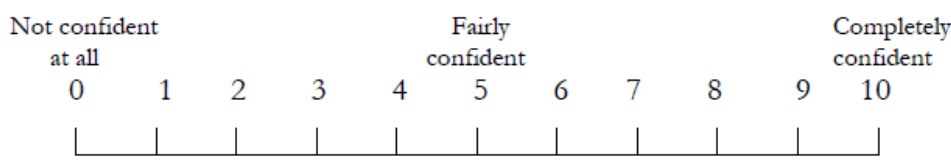

1. Get dressed and undressed

2. Prepare a simple meal

3. Take a bath or a shower

4. Get in/out of a chair

5. Get in/out of bed

6. Answer the door or telephone

7. Walk around the inside of your house

8. Reach into cabinets or closet

9. Light housekeeping

10. Simple shopping

11. Using public transport

12. Crossing roads
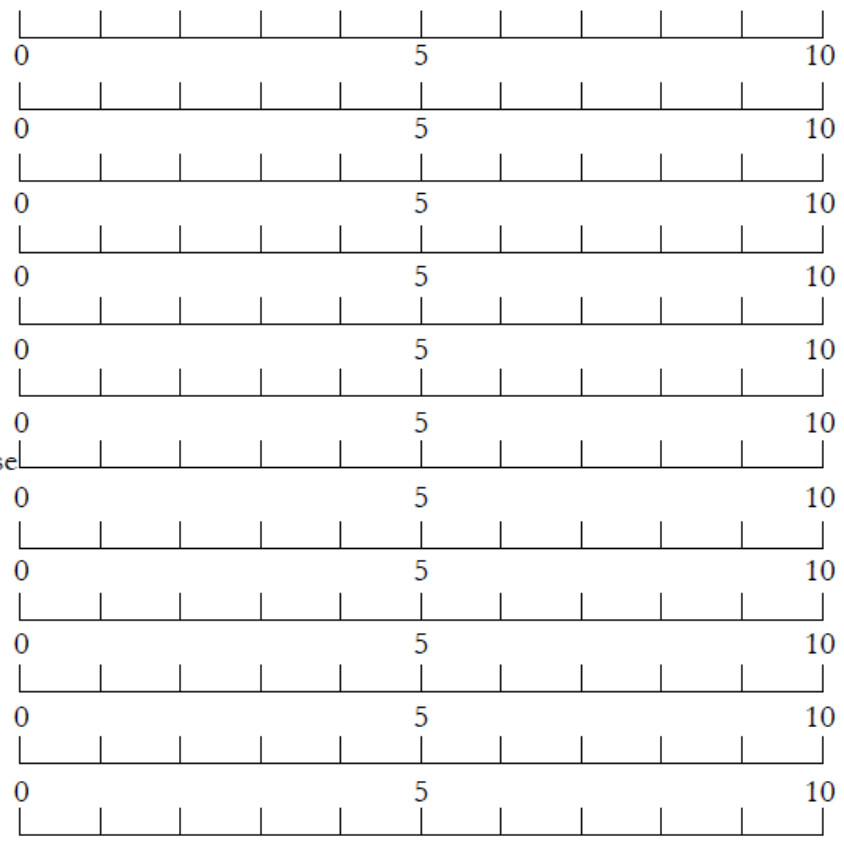

13. Light gardening or hanging out the washing*

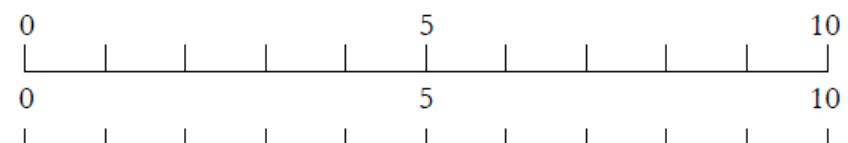

14. Using front or rear steps at home

* rate most commonly performed of these activities

Figure A.13: Modified Falls Efficacy Scale (MFES). Available on: [122].

Table A.1: FSQ Warning Zones [125].

\begin{tabular}{|c|c|c|}
\hline Parameter & Warning Zone & Good \\
\hline \hline 1. Basic activities of daily living & $0-87$ & $88-100$ \\
\hline 2. Intermediate activities of daily living & $0-77$ & $78-100$ \\
\hline 3. Mental health & $0-70$ & $71-100$ \\
\hline 4. Work performance & $0-78$ & $79-100$ \\
\hline 5. Social activities & $0-78$ & $79-100$ \\
\hline 6. Quality of interactions & $0-69$ & $70-100$ \\
\hline
\end{tabular}

\section{A.3.6 The Barthel Index (BI)}

The Barthel Index is a popular ADL scale that was designed to reflect a patient's dependency when performing these activities [126, 127]. It was introduced in 1955 and originally called Maryland Disability Index [127].

It comprises 10 items, each one representing a different activity. Each score is added and the higher the total score, the lower the dependence in ADL [127]. 
Screening tools

\begin{tabular}{|c|c|}
\hline Category & Item \\
\hline \multicolumn{2}{|c|}{ Physical function: During the past month have you had difficulty: } \\
\hline Basic activities of daily living (ADL) & $\begin{array}{l}\text { Taking care of yourself, that is, eating, dressing or bathing? } \\
\text { Moving in and out of a bed or chair? } \\
\text { Walking indoors, such as around your home? }\end{array}$ \\
\hline Intermediate ADL. & $\begin{array}{l}\text { Walking several blocks? } \\
\text { Walking one block or climbing one flight of stairs? } \\
\text { Doing work around the house such as cleaning, light yard work, home maintenance? } \\
\text { Doing errands. such as grocery shopping? } \\
\text { Driving a car or using public transportation? } \\
\text { Doing vigorous activities such as running. lifting heavy objects or participating in strenuous sports? }\end{array}$ \\
\hline
\end{tabular}

Responses: usualiy did with no difficulty (4). some difficulty (3). much difficuity (2), usually did not do because of health (1), usually did not do for other reasons $(0)$.

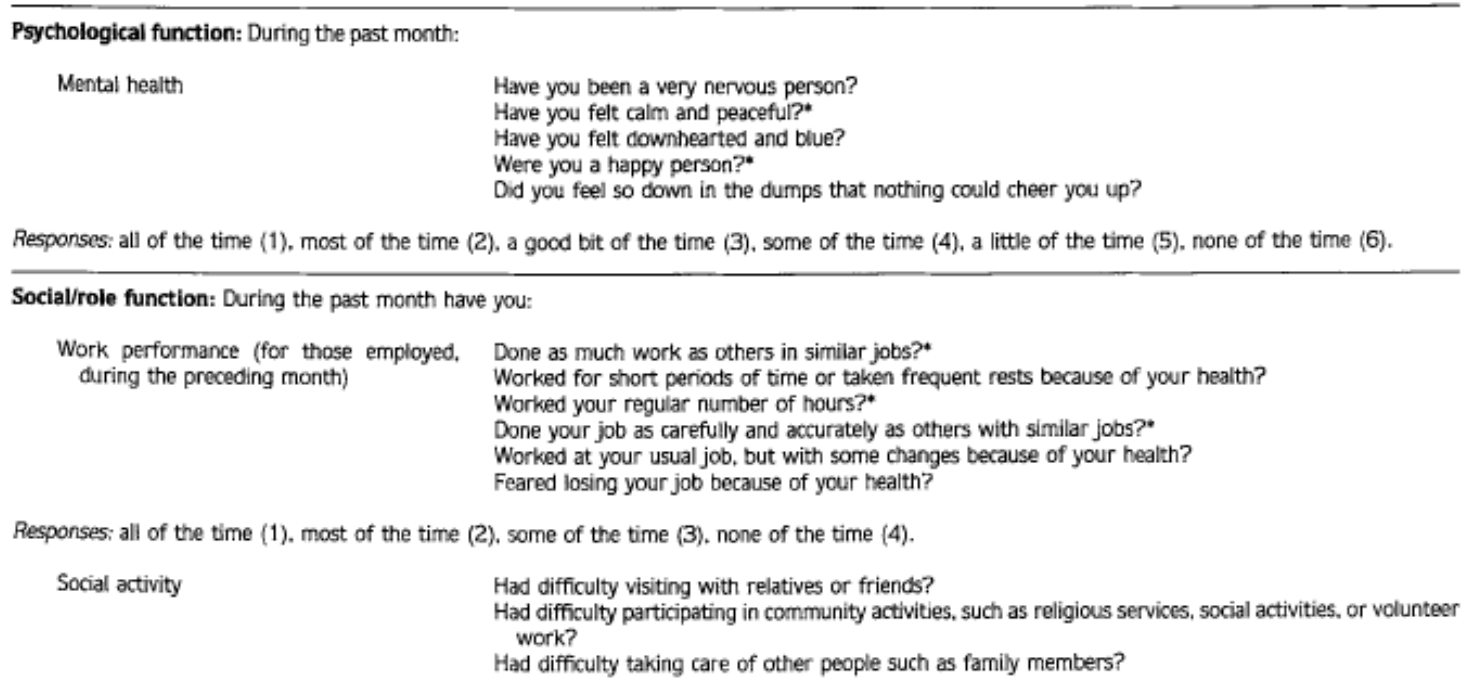

Responses: usually did with no difficulty (4), some difficulty (3). much difficulty (2), usually did not do because of health (1). usually did not do for other reasons (0).

$\begin{array}{ll}\text { Quality of interaction } & \text { Isolated yourself from people around you? } \\ \text { Acted affectionate toward others?* } \\ \text { Acted irritable toward those around you? } \\ \text { Made unreasonable demands on your family and friends? } \\ \text { Gotten along well with other people?*}\end{array}$

Responses: all of the time (1). most of the time (2). a good bit of the time (3). some of the time (4). a little of the time (5). none of the time (6).

\section{Single ftem questions:}

Which of the following statements best describes your work situation during the past month? Responses: working full-time; working part-time; unemployed. looking for work: unemployed because of my health; retired because of my health; retired for some other reason.

During the past month, how many days did illness or injury keep you in bed all or most of the day? Respanse: 0-31 days.

During the past month, how many days did you cut down on the things you usually do for one-half day or more because of your own illness or injury? Response: $0-31$ days.

During the past month, how satisfled were you with your sexual relationships? Responses: very satisfied, satisfied, not sure, dissatisfied, very dissatisfied, did not have any sexual relationships.

How do you feel about your own health? Responses: very satisfied, satisfied, not sure, dissatisfied, very dissatisfied.

During the past month, about how often did you get together with friends or relatives, such as going out together. visiting in each other's homes, or talking on the teiephone? Responses: every day. several times a week. about once a week, two or three times a month, about once a month. not at ali.

*Scores are reversed.

Figure A.14: Functional Status Questionnaire (FSQ). Source: [123].

BI can be administered by observing, testing the patient performing ADL or by selfreport of dependency [127]. The values assigned to each item are based on the time and 
amount of physical assistance that are required if the patient is not able to perform the activity [128].

The Barthel Index is presented in Figure A.15.

\begin{tabular}{|c|c|c|c|}
\hline & & With Help & Independent \\
\hline 1. & Feeding (if food needs to be cut up = help) & 5 & 10 \\
\hline 2. & $\begin{array}{l}\text { Moving from wheelchair to bed and return } \\
\text { (includes sitting up in bed) }\end{array}$ & $5-10$ & 15 \\
\hline 3. & $\begin{array}{l}\text { Personal toilet (wash face, comb hair, shave, } \\
\text { clean teeth) }\end{array}$ & 0 & 5 \\
\hline 4. & $\begin{array}{l}\text { Getting on and off toilet (handling clothes, } \\
\text { wipe, flush) }\end{array}$ & 5 & 10 \\
\hline 5. & Bathing self & 0 & 5 \\
\hline 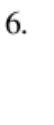 & $\begin{array}{l}\text { Walking on level surface (or if unable to } \\
\text { walk, propel wheelchair) } \\
\text { *score only if unable to walk }\end{array}$ & $0 *$ & $5^{*}$ \\
\hline & Ascend and descend stairs & 5 & 10 \\
\hline 8. & Dressing (includes tying shoes, fastening fasteners) & 5 & 10 \\
\hline & Controlling bowels & 5 & 10 \\
\hline & Controlling bladder & 5 & 10 \\
\hline
\end{tabular}

Figure A.15: The Barthel Index (BI). Source: [128].

\section{A.3.7 Katz Index of Activities of Daily Living (Katz ADL)}

The Katz ADL is a fast and easy to use tool that measures the person's ability to perform basic ADLs independently. It is composed by six items corresponding to six activities, i.e. bathing, dressing, toileting, transferring, continence and feeding. For each of these activities, the person is scored as 1 (if the activity can be performed independently) or 0 (if it cannot be performed independently). A total score of 6 is indicative of full function, but a score of 2 or less indicates severe functional impairment [19, 100].

Although no formal reliability and validity reports could be found on the literature, the tool has been used extensively under clinical and home environments to signalize the non-autonomous functional capabilities [100].

It is recognized that the measurement of these activities at home environment would provide valuable information for adapting the home environment to the abilities of the older person [19]. 
Screening tools

\begin{tabular}{l|l|c|c}
\multicolumn{1}{c|}{ Activity } & \multicolumn{1}{|c}{ Definition of activity independence } & \multicolumn{1}{|c}{ Independent } \\
Bathing & Bathes self completely or needs help in bathing only a single part of the body & 1 & 0 \\
\hline Dressing & $\begin{array}{l}\text { Gets clothes from closets and drawers and puts on clothes and outer garments } \\
\text { complete with fasteners (may have help tying shoes) }\end{array}$ & 1 & 0 \\
\hline Toileting & $\begin{array}{l}\text { Goes to toilet, gets on and off, arranges clothes, cleans genital area without help } \\
\text { (may use an object for support such as a cane or a walker, may use bedpan or } \\
\text { commode during the night) }\end{array}$ & 1 & 0 \\
\hline Transferring & $\begin{array}{l}\text { Moves in and out of bed or chair unassisted (mechanical transfer aids are accep- } \\
\text { table) }\end{array}$ & 1 & 0 \\
\hline Continence & $\begin{array}{l}\text { Exercises complete self control over urination and defecation (occasional accidents } \\
\text { may be ignored) }\end{array}$ & 1 & 0 \\
\hline Feeding & $\begin{array}{l}\text { Gets food from plate into mouth without help (preparation of food may be done by } \\
\text { another person) }\end{array}$ & 1 & 0 \\
\hline & \multicolumn{1}{c}{ Score } & &
\end{tabular}

Figure A.16: Katz ADL. Source: [19].

\section{A.3.8 Lawton Instrumental Activities of Daily Living (Lawton IADL)}

Lawton IADL scale was developed in 1969 by Lawton and Brody to assess the more complex ADLs that are necessary for living in community, as such as shopping, cooking, self-medicating and managing finances [129].

It contains eight items (Figure A.17, and takes 10 to 15 minutes to administer. The summed score range from 0 (total dependence) to 8 (complete autonomy for IADL) [19, 129].

Lawton IADL can be answered by patient, a family member or a caregiver and can be administered with a written questionnaire or by interview. It is appropriate for use in different type of settings, including community-dwelling elderly people [129].

\section{A.3.9 Health Status Questionnaire (HSQ)}

The HSQ (Figure A.18) was used by [63] as a self-report questionnaire, aimed to identify the presence of intrinsic and extrinsic fall risk factors, including, history of falling, limitations in mobility, ADL and IADL, medication use, diseases, dizziness, muscle weakness, pain, mental status, fear of falling and self-efficacy.

The presence of four or more risk factors enabled the categorization of the individuals at high risk of falling [63].

\section{A.3.10 Self report version of the HOME-FAST (HOME FAST-SR)}

Home Falls and Accidents Screening Tool (HOME FAST) is a clinical tool dependent on health professional ratings, aiming to identify environmental hazards. The self report version of HOME FAST enables older people to identify their own risks [26].

It is a yes/no questionnaire, consisting of 87 items. The development of the questionnaire is described in [26]. The complete scale is available online at [130]. 
Screening tools

\begin{tabular}{|c|c|}
\hline Activity & Score \\
\hline \multicolumn{2}{|l|}{ 1. Ability to use telephone } \\
\hline Operates telephone on own initiative; looks up and dials numbers & 1 \\
\hline Dials a few well-known numbers & 1 \\
\hline Answers telephone, but does not dial & 1 \\
\hline Does not use telephone at all & 0 \\
\hline \multicolumn{2}{|l|}{ 2. Shopping } \\
\hline Takes care of all shopping needs independently & 1 \\
\hline Shops independently for small purchases & 0 \\
\hline Needs to be accompanied on any shopping trip & 0 \\
\hline Completely unable to shop & 0 \\
\hline \multicolumn{2}{|l|}{ 3. Food preparation } \\
\hline Plans, prepares, and serves adequate meals independently & 1 \\
\hline Prepares adequate meals if supplied with ingredients & 0 \\
\hline Heats and serves prepared meals or prepares meals but does not maintain adequate diet & 0 \\
\hline Needs to have meals prepared and served & 0 \\
\hline \multicolumn{2}{|l|}{ 4. Housekeeping } \\
\hline Maintains house alone with occasional assistance (heavy work) & 1 \\
\hline Performs light daily tasks such as dishwashing, bed making & 1 \\
\hline Performs light daily tasks, but cannot maintain acceptable level of cleanliness & 1 \\
\hline Needs help with all home maintenance tasks & 1 \\
\hline Does not participate in any housekeeping tasks & 0 \\
\hline \multicolumn{2}{|l|}{ 5. Laundry } \\
\hline Does personal laundry completely & 1 \\
\hline Launders small items, rinses socks, stockings, etc. & 1 \\
\hline All laundry must be done by others & 0 \\
\hline \multicolumn{2}{|l|}{ 6. Mode of Transportation } \\
\hline Travels independently on public transportation or drives own car & 1 \\
\hline Arranges own travel via taxi, but does not otherwise use public transportation & 1 \\
\hline Travels on public transportation when assisted or accompanied by another & 1 \\
\hline Travel limited to taxi or automobile with assistance of another & 0 \\
\hline Does not travel at all & 0 \\
\hline \multicolumn{2}{|l|}{ 7. Responsibility for own medications } \\
\hline Is responsible for taking medication in correct dosages at correct time & 1 \\
\hline Takes responsibility if medication is prepared in advance in separate dosages & 0 \\
\hline Is not capable of dispensing own medication & 0 \\
\hline \multicolumn{2}{|l|}{ 8. Ability to handle finances } \\
\hline $\begin{array}{l}\text { Manages financial matters independently (budgets, writes checks, pays rent and bills, goes to bank); } \\
\text { collects and keeps track of income }\end{array}$ & 1 \\
\hline Manages day-to-day purchases, but needs help with banking, major purchases, etc. & 1 \\
\hline Incapable of handling money & 0 \\
\hline Score & \\
\hline
\end{tabular}

Figure A.17: Lawton Instrumental Activities of Daily Living (Lawton IADL). Source: [19]. 
Screening tools

ID

Date

\section{Health Status Questionnaire}

Demographic Information

Age

Gender $\quad \overline{\mathrm{M} / \mathrm{F}}$

\section{Fall Risk Factors}

Fall history within previous year (an unintentional change in position resulting in coming to rest on the ground or at a lower level)

$$
0-1 \text { fall } \quad \geq 2 \text { falls } \quad \mathrm{Y} / \mathrm{N}
$$

Mechanism of fall

Mobility and ADL Limitations

ADLs / IADLs difficulties (dressing, toileting, bathing,

homemaking, yard work, other

$\mathrm{Y} / \mathrm{N}$

Gait / balance difficulties

$\mathrm{Y} / \mathrm{N}$

Uses ambulation device

$\mathrm{Y} / \mathrm{N}$

Sensory loss on plantar surface of feet

$\mathrm{Y} / \mathrm{N}$

Physically inactive ( $<5$ days/wk, mod activity, accumulated 30

min daily or $<3$ days/wk, vigorous activity, 30 min duration) $\quad \mathrm{Y} / \mathrm{N}$

Medication Use

Polypharmacy (four or more medications) $\quad \mathrm{Y} / \mathrm{N}$

Cardiovascular system medications (diuretics, anithypertensives) $\quad \mathrm{Y} / \mathrm{N}$

Psychoactive medications (sedatives, antidepressants) $\quad \mathrm{Y} / \mathrm{N}$

Musculoskeletal system medications (narcotics, corticosteroids) $\quad \mathrm{Y} / \mathrm{N}$

Other (hypoglycemics, allergy, cold medications___ $\quad \mathrm{Y} / \mathrm{N}$

Medical Conditions

Musculoskeletal (arthritis...)

$\mathrm{Y} / \mathrm{N}$

Neurological (stroke, Parkinson's...) $\quad \mathrm{Y} / \mathrm{N}$

Diabetes $\quad \mathrm{Y} / \mathrm{N}$

Dizziness

Heart disease (postural hypotension, arrhythmias, unstable...) $\quad \mathrm{Y} / \mathrm{N}$

Muscle weakness

Pain

Cognitive status (orientation to person, place, date and time)

SBT if indicated $\quad / 28$

$\mathrm{Y} / \mathrm{N}$

$\mathrm{Y} / \mathrm{N}$

$\mathrm{Y} / \mathrm{N}$

$\mathrm{Y} / \mathrm{N}$

Fear of falling (Have you been a fraid that you might fall inside or outside the home?)

Self Efficacy (Does fear of falling limit yours activities?)

\section{Perceived Health Status}

In general, would you say your health is:

\section{Fall Risk}

Excellent Good Fair Poor

Low risk High risk ( $\geq 4$ risk factors)

Figure A.18: Health Status Questionnaire (HSQ). Source: [63]. 